Portland State University

PDXScholar

Spring 5-23-2018

\title{
Fungal Endophytes in a Seed-Free Host: New Species that Demonstrate Unique Community Dynamics
}

Brett Steven Younginger

Portland State University

Follow this and additional works at: https://pdxscholar.library.pdx.edu/open_access_etds

Part of the Biology Commons, and the Fungi Commons Let us know how access to this document benefits you.

\section{Recommended Citation}

Younginger, Brett Steven, "Fungal Endophytes in a Seed-Free Host: New Species that Demonstrate Unique Community Dynamics" (2018). Dissertations and Theses. Paper 4387.

https://doi.org/10.15760/etd.6271

This Dissertation is brought to you for free and open access. It has been accepted for inclusion in Dissertations and Theses by an authorized administrator of PDXScholar. Please contact us if we can make this document more accessible: pdxscholar@pdx.edu. 
Fungal Endophytes in a Seed-Free Host: New Species That Demonstrate Unique

Community Dynamics

by

Brett Steven Younginger

A dissertation submitted in partial fulfillment of the requirements for the degree of

Doctor of Philosophy

in

Biology

Dissertation Committee:

Daniel J. Ballhorn, Chair

Mitchell B. Cruzan

Todd N. Rosenstiel

John G. Bishop

Catherine E. de Rivera

Portland State University

2018 
(C) 2018 Brett Steven Younginger 


\begin{abstract}
Fungal endophytes are highly diverse, cryptic plant endosymbionts that form asymptomatic infections within host tissue. They represent a large fraction of the millions of undescribed fungal taxa on our planet with some demonstrating mutualistic benefits to their hosts including herbivore and pathogen defense and abiotic stress tolerance. Other endophytes are latent saprotrophs or pathogens, awaiting host plant senescence to begin alternative stages of their life cycles. Most, however, are likely plant commensals with no observable benefits to their hosts while under study. Yet, when considering the contextdependence that may determine plant resistance to pathogen attack, the consortium of endophytes present in the host could alter these outcomes, thereby affecting plant evolution. Despite the excitement of exploiting endophytes for their potential to produce bioactive compounds that are useful to medicine and agriculture, fungal endophyte community ecology is a lagging field. Much remains unknown regarding their colonization patterns within hosts, their spatial and temporal turnover and their diversity. Further, a severe deficiency exists in work on endophytes in seed-free plants, particularly ferns. Since ferns exist in free-living forms in both the haploid and diploid stages, are the second largest group of vascular plants, occupy important ecological niches and represent an older lineage of land plants, this is a major shortcoming in our understanding of plantfungal ecology and evolution. For these reasons, I have examined endophyte community ecology in a widespread fern host in the Pacific Northwest, Polystichum munitum, addressing several gaps in the endophyte literature. Since an understanding of the degree of early endophyte colonization, temporal turnover and the relative contribution of priority effects to community composition are unknown, I conducted a temporal survey
\end{abstract}


of fern endophyte communities utilizing culture-independent, next-generation sequencing on a monthly basis for an entire growing season. A high degree of temporal turnover was observed early in the growing season, where a late colonist rapidly took over the fern population and persisted throughout the year. This colonist, which was isolated from several of the same plants, appears to support growth rates of the host plant when in the gametophytic stage, but is not highly competitive against other endophytes in vitro. Additionally, in an effort to examine host and habitat specificity I examined the spatial turnover of endophytes across four distinct habitat types: a coastal site, a coniferous understory site, a montane site near Mount Saint Helens but not impacted by the 1980 eruption, and a secondary succession site at Mount Saint Helens, spanning 150-km at a single point in time. A high degree of host specificity was found when compared to endophyte communities in neighboring plant taxa and a lack of distance decay was also observed contrasting with other work examining endophyte biogeographic patterns. We conclude that a high degree of host filtering, combined with an abundance of senescent fern material around the base of the plant—which potentially serves as a reservoir of endophyte inoculum - is likely responsible for the observed results. In the process of the ecological studies described above, I isolated over 500 strains of endophytes that corresponded to ca. 100 operational taxonomic units (OTUs). Four of these OTUs are previously undescribed and form a new family and genus, Catenosporaceae and Catenospora, respectively. One of these taxa is responsible for the strong spatial and temporal signals found in the ecological studies. We emphasize that future work should examine if the same phenomena are observed in other fern systems and further encourage endophyte researchers to expand the scope of their investigations into non-traditional 
plant lineages, as exciting ecological interactions that contribute to our understanding endophyte ecology — and community ecology as a whole — are waiting to be discovered. 


\section{Dedication}

This dissertation is dedicated to my wife, Sara Grace Younginger, whose incredible patience and support made this work possible 


\section{Acknowledgements}

I find it highly fitting that the acknowledgements section is most often written last in scientific writing, yet the people acknowledged in these sections are the ones that allow the work to first come into existence. First, I would like to acknowledge that I feel incredibly grateful and fortunate to be able to pursue a doctoral degree in a field for which I hold a strong passion. Many intelligent and talented individuals have not had this opportunity simply because of happenstance and I want it to be known that I do not take this good fortune lightly. Rather, I know it comes with a degree of responsibility to ensure that others may also be given an opportunity to pursue what they love if I am ever in a position to be able to do so.

Secondly, a sincere debt of gratitude is certainly owed to my advisor, Daniel J. Ballhorn. His intellectual, emotional and financial support has been a tremendous asset to my success. He is always willing to take the time to help with both writing and research problems and my decision to join his group has been one that I will never regret. I know that we will remain in contact and look forward to future collaborations together as colleagues. I will always value him as a friend and mentor. To my committee, including Mitch Cruzan, Todd Rosenstiel, John Bishop, and Catherine de Rivera, I thank you. You each have challenged me in different ways and have taken the time out of your busy careers to help make me a better scientist. Thanks to the following funding and permitting agencies: The Botanical Society of America, the International Society of Chemical Ecology, the Forbes-Lea Endowment, the National Science Foundation, Oregon State Parks, and the U.S. Forest Service. 
The research support that I have received from undergraduates has been tremendously helpful. I could not have accomplished this work alone, at least in a timely manner. Specifically, many thanks go out to Meredith Dinsdale, Ashley Davis, Jessie May Laumann, Damas Gawaski, Raymond Whiteman, David Farmer, Sebastian Singleton, JG Bradner, Jahmyl Essex and Margo Craig. For writing support and sincere friendship, I would like to thank Amy Truitt and Lindsay Holden. The company you keep can make all the difference and having you both in my life has been a tremendous asset. I hope I at least partially supported you both from time to time, as well. Also, a huge thank you goes out to my current and former lab mates, especially Adrienne Godschalx and Emily Wolfe. Sincere thanks are due to Dagmara Sirova (Dasa) for the fun field and bench adventures that we've shared during your brief tenure as a Fullbright Postdoc with our group. To Steffi Kautz, I am truly thankful for the time you took to get me started on much of the molecular work required for these projects. To Diane Bland and the herbarium team, I truly appreciate your assistance with voucher specimens and teaching support. For Mehmet, our lab manager, thank you for all of the support, both in research and life transitions. Your molecular and ecological knowledge has been a boon to my research and to everyone else's in our group and I wish you the greatest achievements as you also pursue your doctoral degree. To Nathan I give many, many thanks. We have worked tirelessly together in the field and lab to complete the work contained in this dissertation. Specifically, thank you for your tremendous assistance with culture maintenance and sequencing, phylogenetics support and the many hours spent on missions too numerous to individually recount here. I know you will accomplish exciting 
work in your own dissertation and career and want you to know that I am rooting you on from wherever I am right now.

To my mom, I thank you and love you. The tremendous sacrifices that you made as a single parent to ensure that we were well cared for and loved has helped to shape me into a loving and compassionate father and husband and a tremendously dedicated scientist. And to Sara, my loving and brilliant wife: the most thanks of all go to you. We have been on this dissertation journey together for many years and I know that you share my excitement as it nears its end. You have put your career aspirations on hold while raising our beautiful son and have given me emotions of love and contentment that I had never known prior to meeting you. Thanks for your help in the field and in the lab, willingness to listen to me vent after a long day and incredible patience and sacrifice while I have been in graduate school. I know your own professional life will be incredibly exciting and fulfilling and I can't wait to begin returning the favor whenever you need me. 
$\begin{array}{lc}\text { Abstract } & \text { i } \\ \begin{array}{l}\text { Dedication } \\ \text { Acknowledgements }\end{array} & \text { iv } \\ \begin{array}{l}\text { List of Tables } \\ \text { List of Figures }\end{array} & \text { x } \\ \begin{array}{l}\text { Chapter 1 } \\ \text { Introduction }\end{array} & 1 \\ \begin{array}{l}\text { Chapter } 2 \\ \begin{array}{l}\text { Stable coexistence or competitive exclusion? Fern endophytes defy our understanding } \\ \text { of microbial temporal turnover }\end{array}\end{array}\end{array}$

Chapter 3

Habitat filtering leads to distinct endophyte communities in ferns over a spatial gradient

Chapter 4

Catenosporaceae fam. nov., to include Catenospora gen. nov.; fungal endophytes living in ferns and alder in the Pacific Northwest of North America

Chapter 5

Discussion

References

105

Appendix A

Neighboring plant taxonomy from Chapter 3. Each number corresponds a neighboring plant to each $P$. munitum host sampled.

Appendix B

PCR primers, reaction concentrations and cycling parameters for each locus utilized in the phylogenetic analysis of Chapter 4.

Appendix C

Names and accession numbers of the taxa and their respective loci utilized in the narrow analysis of Chapter 4. 
Appendix D

129

Names and accession numbers of the taxa and their respective loci utilized in the wide analysis of Chapter 4. 


\section{List of Tables}

Table 3.1

63

Checkerboard analysis of OTU incidences within each site (not separated by host type) and examined against two null models for significant differences.

Table 3.2

65

Pearson correlation coefficients of the top 10 OTUs within each site or the full dataset calculated from incidence matrices. 


\section{List of Figures}

Figure 1.1

Fungal endophyte hyphae within the intercellular space of the fern host, Polystichum munitum.

Figure 2.1

Species accumulation curves of operational taxonomic unit (OTU) richness of each sampling month in the culture-independent study.

Figure 2.2

Hill measures of alpha diversity at the scales of $q=0,1$, and 2 of each sampling month in the culture-independent study.

Figure 2.3

Non-metric multidimensional scaling (NMDS) of endophyte community composition within each sampling month in the culture-independent study.

Figure 2.4

Taxonomy plot of endophyte community composition within each sampling month of the culture-independent study.

Figure 2.5

Results of in vitro competition assays of OTU1 versus 11 other strains recovered from the same plants examined in the culture-independent study.

Figure 2.6

37

The change in surface area $\left(\mathrm{cm}^{2}\right)$ of $P$. munitum gametophytes treated with conidia suspensions generated from fungal cultures initially isolated from the host plants examined in the culture-independent study (OTU1 and Plectania milleri) or with sterile $\mathrm{DDH}_{2} \mathrm{O}$ (control).

Figure 2.7

Representative images of gametophyte assays.

Figure 3.1

60

Hill measures of alpha diversity at the scales of $\mathrm{q}=0,1$ and 2 within each sampling site, separated by endophyte communities colonizing the Polystichum munitum host and endophytes colonizing the plants growing in closest proximity to the P. munitum host.

Figure 3.2

Non-metric multidimensional scaling (NMDS) of endophyte community composition across the full dataset. 
Figure 3.3

Taxonomy plot of endophyte community composition within site, separated by the Polystichum munitum host and neighboring plants.

Figure 4.1

86

Phylogenetic tree from the narrow analysis showing the relationship of Catenosporaceae to other, closely-related Leotiomycetes.

Figure 4.2

88

Phylogenetic tree from the wide analysis showing the relationship of Catenosporaceae to other, closely-related Leotiomycetes.

Figure 4.3

Colony characteristics of the four spp. nov. on malt extract agar (MEA) and potato dextrose agar (PDA).

Figure 4.4 92

Differential interference contrast images of hypha and conidium morphology of the four spp. nov. on malt extract agar (MEA; C. loowitii) or Triticum grain (all others). 


\section{Chapter 1}

Introduction

The plant microbiota is a ubiquitous component of terrestrial ecosystems, affecting host evolutionary trajectories and global nutrient cycling (Younginger et al. 2009; Moon et al. 2013; Peay and Bruns 2014), and represents a vast reservoir of undiscovered taxa with important ecological functions (O'Brien et al. 2005). It is comprised of many groups of pro- and eukaryotic microorganisms including bacteria, archaea, protists, and fungi (Berg et al. 2016). Within the fungal kingdom, plants are known to host mycorrhizal fungi, which form intimate root associations and exchange water, phosphorus, and nitrogen for host-derived photosynthate (Smith et al. 2003; Harrison 2005), endophytic fungi, which largely reside in intercellular spaces both above- and belowground (Clay 1999; Kiers and Heijden 2006) and pathogenic fungi, the most common type of plant pathogens (Nilsson et al. 2014). Of these groups, fungal endophytes are the most poorly understood ecologically and also the most diverse taxonomically (Sun and Guo 2012); however, over the past three decades, research into fungal endophyte diversity and function has been a burgeoning field due to the excitement garnered around their mutualistic ability within grasses (Sullivan and Faeth 2004; Becker et al. 2016) and their potential to produce bioactive compounds of medical importance (Strobel and Daisy 2003). Despite this excitement, fungal endophyte community ecology—and microbial community ecology in general — substantially lags behind other fields of ecology (Mihaljevic 2012) and much stands to be gained by turning our focus towards this hyperdiverse and ubiquitous group of organisms. 
Fossilized remains of Siluro-Devonian land plants discovered in the Rhynie chert clearly show fungal structures associated with plant cells, leading to speculation that plant-fungal associations are as ancient as land plants themselves (Selosse and Le Tacon 1998; Krings et al. 2007). Since the colonization of terrestrial habitats required Streptophyte algae to tolerate desiccation, phosphorus and nitrogen limitation and intense UV radiation (Becker and Marin 2009), it is currently hypothesized that fungi (already present in aquatic and terrestrial habitats) facilitated the evolution of land plants (Pirozynski and Malloch 1975). Although the origin of the endophytic lifestyle likely evolved simultaneously with land plants, ancestral state reconstructions of Pezizomycotina fungi within the Ascomycota demonstrate that many extant lineages of endophytic fungi evolved from endolichenic fungi that intimately associate with algal cells on the interior of the lichen thallus (Arnold et al. 2009). Since terrestrial habitats were likely to first be colonized by lichens that degraded rocky substrate into finer material more suitable for plants, the origin of endophytic fungi from endolichenic fungi seems likely. Additionally, Arnold's (2009) ancestral state analysis shows frequent transitions from the endolichenic state to the endophytic and pathogenic state, with few transitions from free-living saprotrophic states back to the endophytic. In a separate analysis, Delaye et al. (2013) also finds transitions to and from pathogenic and endophytic states, particularly from necrotrophic fungi. Although the remarkable lifestyle lability of lineages of fungi - and also of extant fungi-will be discussed further, these results provide a tantalizing glimpse of the origins of the endophytic trophic guild. Since plants have evolved in the presence of a microbial world (Gilbert et al. 2012), it makes intuitive sense that they continue to be abundantly colonized by fungi which can affect 
their fitness and evolution. Additionally, only 70,000 fungal species are currently described. Some observations have shown that fungi outnumber plants in natural habitats by a ratio of 6:1, while more recent estimates obtained from high-throughput sequencing place the total number of global fungal taxa at 5.1 million, making only $1-3 \%$ of fungi known to science (Hawksworth and Rossman 1997; Blackwell 2011). This staggering amount of unknown diversity—which includes fungal endophytes—holds many answers to questions about plant ecology, evolution and global nutrient cycling, waiting to be discovered.

Endophytes are currently defined as fungi that form unapparent and asymptomatic infections within plant tissues at the time of study, requiring plant photosythate for survival and emerging from senescent host tissues to sporulate (Wilson 1995; Rodriguez et al. 2009). Most are microfungi and are not known to produce large, multicellular fruiting bodies. Endophytes are typically only observed in their anamorphic (asexual) form as either hyphomycetes which bear free conidiophores (asexual spore-producing structures) directly on their mycelia or coelemycetes, which possess pycnidia, synnema, or acervuli (i.e. blister-like structures that penetrate the plant epidermis to release conidia; Subramanian 1983). Although they may at some point undergo sexual reproduction in senescent plant material (Carroll 1988), the endophytic lifestyle is currently understood to be one of low metabolic activity when living intercellularly (Thomas et al. 2016). Fungal endophytes are currently classified into two main groups: those from the fungal family Clavicipitaceae (Hypocreales of Ascomycota) that colonize temperate grasses and those from the phyla Ascomycota, Basidiomycota and Zygomycota that colonize all other land plants (Rodriguez et al. 2009). The non-grass endophytes are highly diverse (Arnold et al. 
2000), typically horizontally transmitted via fungal sporulation events, water and herbivore vectors, form highly localized infections in all above- and belowground portions of plants, perform ecologically diverse functions in hosts covering the spectrum of symbiotic interactions (Peršoh 2013), and are taxonomically and ecologically poorly understood (Rodriguez et al. 2009). It is the study of these highly diverse, non-grass endophytes that will form the basis of the work described herein. In contrast, more attention has been paid to the taxonomically narrow group of grass endophytes following the discovery by Clay (1988) which demonstrated the ability of Epichloë spp. to produce alkaloids that defend grass hosts from mammalian herbivory. However, Epichloë spp. are unique from other endophytes in that their reproduction is linked to the vertical transmission of their hosts through seeds (Sneck et al. 2017), form systemic infections in shoots and rhizomes, and are highly host-specific (Schirrmann et al. 2014). Since Epichloë spp. infect temperate grasses of agronomic importance for grazing mammals, potentially resulting in significant losses to livestock due to their toxicity (Panaccione et al. 2001), a focus on this narrow group of endophytes is understandable; however, this restricted scope has largely ignored much of the fungal diversity on this planet which is found in non-grass endophytes.

Although grass endophytes demonstrate the impressive ability to produce an array of toxic alkaloids that deter herbivory, the non-grass endophytes also provide benefits to their hosts that are of equal import. The literature contains examples of non-grass endophytes conferring mutualistic benefits including herbivore defense (Ownley et al. 2009; Cook et al. 2013; Vidal and Jaber 2015), pathogen defense (Arnold et al. 2003; Paparu et al. 2007; Mejía et al. 2008; Ownley et al. 2009; Qadri et al. 2014; González- 
Teuber et al. 2014; Busby et al. 2015a; Terhonen et al. 2016; Schlegel et al. 2016;

Christian et al. 2017; McMullin et al. 2018), thermotolerance (Zhou et al. 2015), nutrient acquisition (Almario et al. 2017), growth promotion (Mucciarelli et al. 2003), increased allelopathy (Aschehoug et al. 2014), and enhanced nodulation in leguminous plants (Zhang et al. 2015). Additionally, endophytes can alter a plant's intrinsic defensive mechanisms through a manipulation of phytohormones (Navarro-Meléndez and Heil 2014; Cosme et al. 2016). Despite the ever-growing list of mutualistic benefits that endophytes have been shown to provide to plants, many endophytes likely exist as commensals in natural habitats (Mihaljevic 2012; Delaye et al. 2013; May 2016) relying upon plant photosynthate and providing no apparent benefit in return.

Excitement in the discovery of mutualistic endophytes and a publication bias towards reporting beneficial effects has resulted in a perception that most fungal endophytes provide positive benefits for their hosts (Rodriguez et al. 2004; Hartley and Gange 2009; Dupont et al. 2015). Indeed, since Anton de Bary (1878) first described a symbiotic continuum which encompassed positive, neutral and negative interspecies interactions, we have viewed symbioses with an assumption that they largely remain static regarding the benefits and harms between hosts and symbionts (Oulhen et al. 2016). However, within a single season, endophytic microbes have the ability to become more pathogenic or mutualistic towards their hosts (Fesel and Zuccaro 2016). A newfound appreciation for the highly context-dependent interactions that occur between endophytes and host plants calls for a shift in our understanding of this ubiquitous symbiosis (Busby et al. 2015a). It has been proposed that we assume that most endophytes exist as neutral plant symbionts or commensals that get pushed to either extreme - towards pathogenic or 
mutualistic forms - based upon the prevailing biotic and abiotic conditions that drives their context dependency (May 2016). The definition of commensal may even be unsatisfactory within an empirical framework, as the absence of proof of benefit or harm to a host does not imply that there is proof of its absence and even slightly beneficial or harmful interactions do not exist exactly in the middle of the continuum (Zapalski 2011). Additionally, debate has ensued over whether the holobiont—-the host and its associated microbes - evolves collectively as a hologenome (Bordenstein and Theis 2015) or instead if the two groups of organisms are on separate evolutionary trajectories (Moran and Sloan 2015). However, the former scenario runs counter to our current theoretical understanding of fitness, since minor changes to an individual microbe that results in an increase in fitness should lead to its spread in the community. Although plant symbionts live in intimate associations on and within plant tissues, they are also subjected to their own forms of selection independent of their host's (Friesen 2013).

Transitioning between more parasitic and mutualistic phenotypes within hosts is intriguing, but an even more extreme form of lifestyle lability has been clarified into a formal hypothesis by George Carroll (1999). Termed the "Foraging Ascomycete" hypothesis, it posits that the endophytic trophic guild is just one stage of more commonly observed saprophytic and pathogenic fungi (Carroll 1988). This lability has enabled fungi to exploit the endophytic mode to colonize new habitats, gain an advantage over other saprotrophs when host tissue senesces (i.e. priority effects), disperse to distant sites and potentially tolerate seasons unfavorable to reproduction (Thomas et al. 2016, U'Ren and Arnold 2016, McMullin et al. 2018). It has also been discovered that aquatic hyphomycetes are able to exploit the endophytic trophic guild. A culture-based analysis 
of Picea mariana (black spruce) found fungal endophytes living in host needles that were also aquatic hyphomycetes and produced conidia adapted to freshwater habitats when placed in aerated water chambers (Sokolski et al. 2006). The ability to utilize drastically different trophic strategies (e.g. decomposing immobile lignin and cellulose versus evading host defenses and absorbing photosynthate) is intriguing and provides possible explanations for the breadth of fungal distributions and richness that could be addressed in future ecological studies (Chauvet et al. 2016).

Useful theories have been developed in the field of community ecology through the study of macroorganisms to explain the patterns and causes of species distributions (MacArthur and Wilson 1967; Hubbell 2001; Mouquet and Loreau 2002, 2003; Chase 2003; Vanschoenwinkel et al. 2007; Pedruski and Arnott 2011). As useful as they have proven to be, it is vital that we also examine whether the same frameworks apply to microbial systems. Since macroorganisms initially evolved from microbes, presumably the same theories should apply to both; yet a unique suite of selective pressures applies to microbes, particularly endosymbiotic taxa. Beyond tolerating abiotic conditions, competing with interspecifics, acquiring resources and evading pathogens, endosymbionts must also contend with host biology to colonize and persist. Plants have simultaneously evolved methods to avoid antagonistic microbes which take a greater share of resources, adversely affecting host fitness. Further, microbes may have greater population densities and species richness based on trends observed with the body size of macroorganisms and also trends observed in empirical data on microbes (Martiny et al. 2006). Baas Becking's (1934) "Everything is everywhere, but the environment selects" hypothesis has remained a cornerstone for explaining microbial biogeography for a 
century, predicting that microbial dispersal is high, but habitat filtering is sufficiently large to result in endemism (Queloz et al. 2011). However, this view was initially developed for free-living microbes and although patterns of symbiotic microbial distributions are beginning to emerge in some work (e.g. dispersal limitation and correlations with elevation, precipitation and nutrient availability; (Jumpponen and Jones 2010; Zimmerman and Vitousek 2012; Meiser et al. 2014), the field is far from a consensus on the major drivers of endophytic spatial distributions.

One important factor that may contribute to endosymbiont biogeographic patterns is whether host-imposed filtering plays a greater role in determining community composition than more traditional stochastic and deterministic drivers like dispersal, competition, and environmental filtering. If so, this may result in a greater degree of hostspecificity for particular taxa, though this is not clarified in the current literature. Theory predicts that interspecific co-existence may occur more readily in stressful habitats (Bertness and Callaway 1994; Castro et al. 2013), but it is unclear if this is the case for the intercellular spaces of hosts. Conversely, host tissue may provide a buffer to abiotic stressors and instead be a highly competitive place to co-exist. One survey of endophyte distributions from the arctic to the tropics found a mix of host-specific to generalist relationships, but also failed to find broadly-ranging taxa, though this study relied upon culture-based methods and the existence of uncultivable taxa could have hindered conclusions (Arnold and Lutzoni 2007). Although we are far from a clear understanding of how host filtering interacts with abiotic variables to affect endophyte community composition, if host plants facilitate endophyte colonization or buffer against abiotic stressors, a strong host-dependent signal is likely to be observed across widely varying 
habitat types where dispersal is sufficient. This is also vital to understand from an applied sense as beneficial microbes may succeed in highly specific abiotic conditions (the same as where they were isolated/tested) but could perform poorly when transported to more distant sites. Moreover, many organisms do not find all seasons or periods of time favorable to growth or persistence (Chesson and Huntly 1997), yet there is no clear evidence describing the degree of temporal turnover of fungal endophytes on a fine scale. Since there is a strong desire to inoculate agricultural plants with beneficial microbes (Gundel et al. 2013; Busby et al. 2017), this is critically important to uncover as management efforts may not be effective if dynamic, pre-existing microbial communities swamp the effects of managed treatments. 


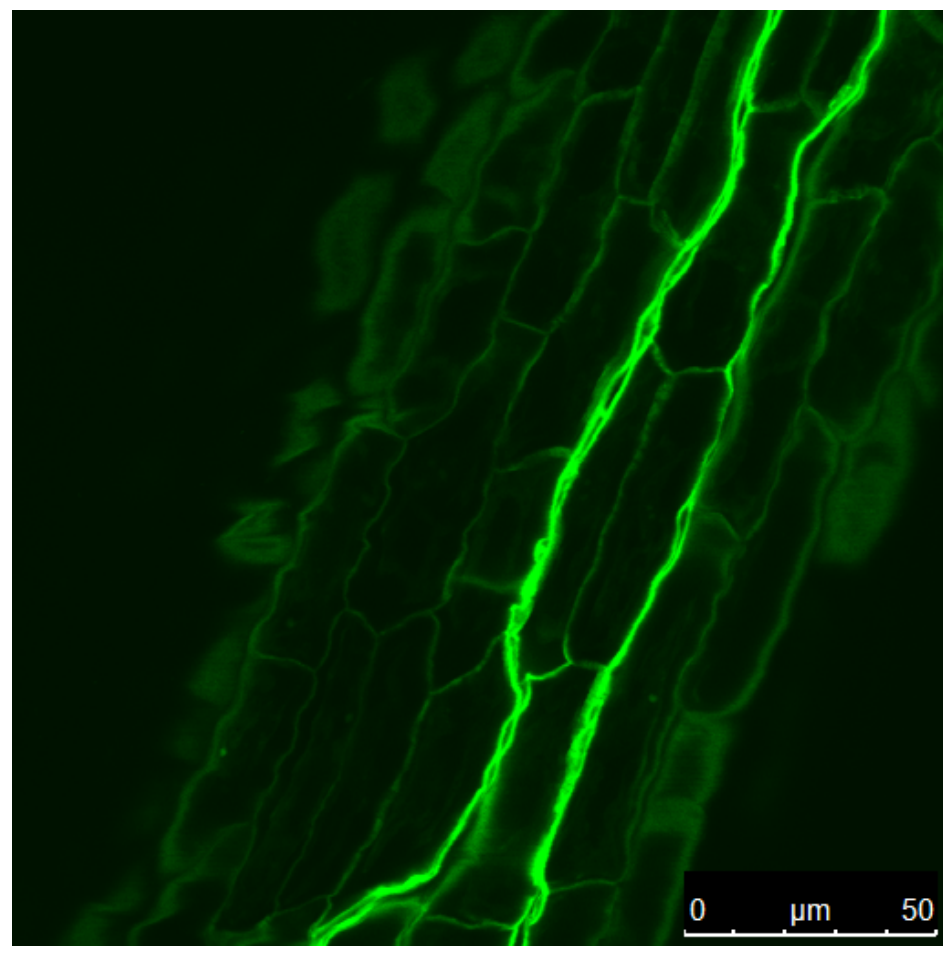

One other major

deficiency in the study of

fungal endophytes is the focus

on non-angiosperm plant

lineages. Although progress has

been made in studies centered

on fern endophytes over the

past decade, (Zubek et al. 2010;

Del Olmo-Ruiz and Arnold

2017; Younginger and Ballhorn

2017) the field is substantially

Figure 1.1 Fungal endophyte hyphae within the apoplast of the fern host, Polystichum munitum. Image generated at $430 \mathrm{X}$ with $405 \mathrm{~nm}$ excitation and scanning at $411-485 \mathrm{~nm}$. Fungal hyphae stained with Uvitex 2B.

lagging behind angiosperm

endophyte research (Figure

1.1). Since ferns are not economically exploited and we tend to view plant distributions

from the perspective of angiosperms, this deficiency is understandable; however, it could be argued that this is an incredible oversight which is limiting the progress of the field.

Ferns and other seed-free plants are unique in their manner of reproduction, relying upon spores for dispersal and possessing haploid gametophytes that are subjected to strong levels of selection in nature (Watkins et al. 2007). This could present an additional stage of plant growth for endophytes or pathogens to exploit, thereby increasing the likelihood for novel plant strategies to avoid deleterious interactions (Swatzell et al. 1996). Also, ferns are certainly ecologically important plants occupying many niches in both climax and disturbed communities (Mehltreter et al. 2010), and are particularly apparent 
epiphytic plants in the tropics (Little and Barrington 2003) and understory habitats in the Pacific Northwest. Further, they are the second-most species rich group of vascular plants behind the angiosperms, presenting opportunities for unique, host-specific taxa and plantmicrobe interactions. Finally, they represent a lineage of plants that predates seed plants and may have evolved unique strategies for tolerating and associating with ubiquitous fungal microbes, though most extant ferns are more recently derived alongside the angiosperms (Schuettpelz and Pryer 2009). For these reasons, I have chosen to focus on fungal endophyte communities in a highly abundant fern host in the Pacific Northwest, Polystichum munitum (western swordfern). P. munitum is an ideal plant to study endophyte community ecology for several reasons: It tolerates a broad range of abiotic conditions including damp and shaded coniferous understories, coastal habitats subjected to salt spray and intense ultraviolet (UV) radiation and montane habitats additionally subjected to UV, extreme temperature fluctuations, seasonal inundation in snow and desiccation in the summer months. Additionally, it is an evergreen plant that may serve as important habitat for endophytes in the winter months. This provides opportunities to investigate both spatial and temporal dynamics of endophyte communities in a single host plant species across a large geographic range. By examining endophytes in drastically different habitat types or seasons, interesting discoveries about unique microbial colonists may emerge. Furthermore, because it is a spore-reproducing plant, it is particularly useful for the generation of sterile gametophytes in culture through surface sterilization, allowing for examinations of the functional benefits of endophytes at the haploid stage of the host. Finally, by focusing on fern endophytes, I am contributing to filling a significant 
gap in our knowledge of the ecology, taxonomy and natural history of ubiquitous and diverse fungi that colonize understudied plant lineages.

When considering the lack of a formal understanding of endophyte spatial and temporal patterns in natural settings, especially in seed-free plant lineages, I have chosen to investigate endophyte communities in P. munitum through three interrelated chapters by addressing (i) their temporal dynamics at a single study site over an entire growing season (ii) their spatial turnover and degree of host-specificity in four distinct habitats spanning a 150-km range and (iii) whether any unique, undescribed taxa associate with the fern host in our region.

For the first chapter, I examined the temporal turnover of fungal endophytes in a single host population. Although previous work has surveyed temporal changes in fungal endophytes within a single host (Suryanarayanan and Thennarasan 2004), no work has yet examined them on a fine scale for an entire growing season utilizing cultureindependent methods. It is important to clarify whether plants are colonized early (soon after leaves emerge in the spring) and how important priority effects are for community composition in later months. Also, clarifying the beta diversity of endophyte communities in a single host over time will provide pivotal information on whether a stable equilibrium predominates with multiple taxa co-existing, or instead if competitive exclusion prevails. We know that free-living microbial communities can be dynamic, especially in a changing world, but how this dynamic nature compares with the natural fluctuations of endosymbiotic organisms in a typical season is unknown. It is also crucial to leverage culture-independent methods to examine endophyte community composition, since only a small fraction of endophytes can be grown on artificial media (Sun and Guo 
2012), limiting our inferences to a narrow group of fast-growing resource generalists. Advancements in next-generation sequencing (NGS) technology have enabled us to further our understanding of the likelihood of millions of eukaryotic fungi living on and within plants. Understanding why and how such a staggering amount of diversity persists is of paramount importance. With this in mind, I address four main questions for the first component of this work: (i) Do priority effects play an important role in structuring fungal endophyte communities in host plants? (ii) Do endophyte communities demonstrate significant temporal turnover throughout the growing season? (iii) Are endophyte community dynamics different in ferns than in seed plants? (iv) Do any notable community interactions occur, and if so, how do these taxa interact in the presence of competition and affect the host plant? To address these questions, I sampled 20 plants monthly for a period of 10 months. I began the sampling regime when fern leaves first emerged as croziers (i.e. fiddleheads), by sampling an individual pinna (i.e. leaflet) on the same four leaves per plant on a monthly basis. Culture-independent techniques were leveraged for this component, though extensive culturing was also conducted from remaining tissue. Through this study, I discovered a highly abundant endophyte that is mostly absent from newly-emerged host tissue in the spring, but following one month of exposure to the field, quickly takes over the endophyte community and remains in high abundance throughout the rest of the host growing season. I next examined the competitive nature of this abundant endophyte (which we were able to isolate from culturing efforts) in vitro against 11 other endophytes isolated from the same host plants, finding that it is not highly competitive in artificial conditions. Lastly, I examined the effects of this abundant endophyte on the growth rate of 
gametophytes of the P. munitum host, finding that it results in increased growth at the haploid stage, though not significantly different from sterile controls.

For the second chapter, I again leveraged culture-independent techniques to examine the spatial turnover of endophyte communities in four distinct habitats at a single point in time. No study has yet examined the degree of host specificity across several unique habitat types at a single point in time (to the best of my knowledge), accounting for temporal shifts that were shown to occur in the temporal study above. This is vital to examine since a definition of a regional scale for endophyte communities has proven elusive. Likewise, a clarification of whether dispersal limitation/distance decay is truly a phenomenon in this endophyte system will contribute further evidence to our understanding of their biogeographic patterns. I predicted that if host-specificity does exist for particular taxa, it is likely that deterministic processes such as habitat filtering are occurring. Conversely, if there is a high degree of generalism, it is more likely that neutral processes predominate in this system. This is a particularly important point to consider in light of the need for endophytes to overcome host defenses just to colonize, let alone tolerate additional abiotic stressors and competition from interspecific taxa. To clarify the abovementioned spatial-related deficiencies, I asked the following questions: (i) Is significant spatial turnover observed across the four unique sampling sites? (ii) Are there patterns of host-specificity observed in either $P$. munitum or other neighboring plants? (iii) If there is a degree of host specificity, does this result in discernable patterns of competitive exclusion or the aggregation of certain co-occurring taxa? and (iv) What ecological principles may apply to observed patterns of spatial turnover and host specificity? To address these questions, I sampled 10 ferns at each study site, and also 2 
neighboring plants (not $P$. munitum) next to each fern sampled, and sequenced fungal DNA isolated from these tissues with NGS technology. I determined the degree of similarity in endophyte community composition between ferns within a site and between neighbors within a site. I additionally examined the similarity in fern communities across sites. Further, I conducted a co-occurrence analysis using checkerboard scores against null models of community composition to determine if competitive exclusion was occurring at the sites. Finally, I examined whether significant correlations existed between abundant taxa in the dataset in either positive or negative directions to further assess whether exclusion appeared to occur. Surprisingly, I found a significant degree of host specificity for endophytes in P. munitum. Endophyte communities were more similar in fern hosts $150 \mathrm{~km}$ apart than in neighboring plants growing in the immediate vicinity (sometimes even touching) each fern host. A strong checkerboard pattern was observed in three of the four sites, with significant differences from null models, which correlated with contrasts in alpha diversity measures between ferns and neighbors within each site. A strong signal of negative correlations between one highly abundant taxon and other abundant taxa was detected in the ferns. This was determined to be the same abundant taxon discovered in the temporal study. We conclude that a distance decay is not found across the $150-\mathrm{km}$ gradient in this study, and that a high degree of host specificity is found for endophytes within the P. munitum host.

Finally, since this is one of the first comprehensive examinations of endophyte communities in ferns to the best of our knowledge, the potential exists for the discovery of novel taxa. When considering the drastic discrepancy between what we know exists and what we predict exists for fungal taxa (Blackwell 2011), it is important to further 
investigate whether any novel taxa are recovered in culture-based work, particularly if interesting ecological interactions are observed in culture-independent work. While simultaneously conducting sampling efforts for the previous temporal and spatial studies, I have isolated four taxa-including the abundant taxon that plays a significant role in $P$. munitum endophyte community composition—-belonging to a family nova and genus nova. The phylogeny of these four species novae are described based on their unique morphology, monophyly and phylogenetic distance from other known taxa for the fourth chapter. We propose the fam. nov. Catenosporaceae to include the gen. nov. Catenospora and the four spp. nov. C. polysticola, C. gilkeyae, C. carrollii, and C. loowitii.

Taken together, the work pursued herein helps to fill several significant gaps in fungal endophyte community ecology and taxonomy. Not only is this the first examination of endophytes in a single fern host species and the first examination of fern endophyte community composition utilizing culture-independent NGS technology, but it also provides crucial information on both the spatial and temporal dynamics of these cryptic microbes. Specifically, this work demonstrates sharp endophyte temporal turnover with a dominant taxon which persists throughout the growing season, a high degree of host-specificity for fern endophytes across $150 \mathrm{~km}$ and the discovery of four new taxa that comprise a fam. nov. One of these taxa is also confirmed the be same genotype observed to be highly dominant in both the spatial and temporal studies. This work provides concrete evidence of the importance of examining endophyte communities in non-traditional plant lineages, expanding our scope of understanding about ubiquitous microbial symbionts that may be shaping host plant evolution globally. 


\section{Chapter 2}

Stable coexistence or competitive exclusion? Fern endophytes shift our understanding of microbial temporal turnover

Introduction

Recent explorations of the microbial world have revealed that our current understanding of the organism as a biological unit is insufficient, as all macroorganisms are engaged in intimate symbioses with microbes (Gilbert et al. 2012; Bordenstein and Theis 2015). Our newfound awareness of the tremendous bacterial and fungal diversity colonizing multicellular eukaryotes has initiated much excitement regarding their potential functional benefits to hosts (Gundel et al. 2013; Farrar et al. 2014; Busby et al. 2017). The plant microbiome is no exception, with evidence demonstrating the ability of these microbes to promote plant growth (Knoth et al. 2014; Khan et al. 2016), defend against pathogens and herbivores (Busby et al. 2013; Christian et al. 2017), and provide abiotic stress tolerance (Rodriguez et al. 2008; Redman et al. 2011). One such group of plant-associated microbes - fungal endophytes — are microfungi that colonize intercellular host tissues in an asymptomatic manner (Wilson 1995) and are broadly classified according to their mode of transmission between hosts and the tissue types in which they reside (Rodriguez et al. 2009). Current estimates predict the existence of several million undescribed fungal taxa (Hawksworth and Rossman 1997; Blackwell 2011 - most of which are plant-associated; therefore, it is not hyperbole to claim that our current understanding of fungal endophyte diversity, ecology and natural history is scant at best. 
Although a mounting number of studies have demonstrated the existence of mutualistic endophytes under specific conditions, there is a recent acknowledgement that most endophytes are commensals that respond to biotic and abiotic conditions in a context-dependent manner (Busby et al. 2015b; May 2016), thereby limiting their utility in applied settings. Additionally, if we wish to utilize endophytes that have demonstrated a mutualistic phenotype in beneficial applications to hosts, several critical areas must be clarified, including (i) the range of ways they colonize plant tissue and which of these are most common, (ii) what determines their success in planta, and (iii) what enables their persistence through time. Our efforts to inoculate target plants with a chosen microbe will likely be futile if they are unable to colonize, outcompete pre-existing microbes and persist throughout the growing season.

Furthermore, since many microbial colonists cannot be cultured in vitro (Sun and Guo 2012), there is high value in conducting culture-independent analyses of native microbial communities to draw general conclusions about community composition and dynamics (Peay 2014). Of the culture-independent studies that have examined endophyte community composition, they most commonly document community composition at a single point in time (Zimmerman and Vitousek 2012, Bálint et al. 2015, but see Kohout et al. 2013). Although time, labor and financial constraints factor into this reliance on single sampling events, the practice severely limits our understanding of endophyte community dynamics throughout a host's growing season. Previous culture-independent work examining temporal turnover has indeed demonstrated a significant difference in community composition over just two sequential sampling months (Younginger and 
Ballhorn 2017), but work clearly documenting the annual turnover of plant microbial communities is a large deficiency in the current state of the knowledge in the field.

Despite this deficiency, many useful theoretical frameworks have been developed in the field of community ecology (MacArthur and Wilson 1967; Leibold et al. 2004; Urban et al. 2008). With recent advancements in sequencing technologies, we now have the opportunity to determine if these same principles of community ecology apply to microorganisms (Mihaljevic 2012; Borer et al. 2016; Koskella et al. 2017). For example, once microbial colonists arrive to new habitat (i.e. newly emerged tissue)—since most endophytes are horizontally transmitted between hosts (Oono et al. 2014) - it is currently unclear how stochastic processes including priority effects (Chase 2003; Fukami 2015), or deterministic processes such as legacy (Warner and Chesson 1985) and storage effects (Kennedy 2010) interact to drive realized community composition. Further still, the relative importance of competitive interactions within host tissue after colonization occurs deserves much attention due to its high potential to affect species richness and diversity over time.

Another equally important deficiency in the knowledge of plant microbial communities is the lack of work in a broad range of plant systems. Much of the work on endophytes thus far has understandably focused on economically important host plant systems (Clay 1988; Redman et al. 2011; Busby et al. 2013), but countless unique and ecologically important microbial interactions are lying in wait in other understudied plant systems. One such overlooked group, the pteridophytes (ferns and other sporereproducing vascular plants) are the second-largest group of vascular plants that occupy many important ecological niches and possess independent haploid and diploid life stages 
(Schuettpelz and Pryer 2009). We have chosen to utilize a regionally-abundant fern in the Pacific Northwest, western swordfern (Polystichum munitum) due to its evergreen growth and tractability as a host plant system in our region.

In the present work, we examined the temporal turnover of endophyte communities over an entire growing season and further examined microbe-microbe competitive interactions and plant-microbe functional interactions through three main components: a culture-independent observational study, a culture-based competition assay, and a culture-based fern gametophyte assay. We sought to address four main questions: (i) Do priority effects play an important role in structuring fungal endophyte communities in host plants? (ii) Do endophyte communities demonstrate significant temporal turnover throughout the growing season? (iii) Are endophyte community dynamics different in ferns than in seed plants? (iv) Do any notable community interactions occur, and if so, how do these taxa interact in the presence of competition and affect the host plant? The present work is a comprehensive examination of fern microbial communities growing in a coniferous understory in the Coast Range of Western Oregon. We hope the results presented herein will encourage other researchers to determine whether similar phenomena are observable in other endophyte-host plant systems, regions and biomes.

Methods

Study site location and establishment-

The field survey was conducted in the eastern foothills of the Oregon Coast Range at $45.73376 \mathrm{~N}, 123.18631 \mathrm{~W}$ and 310 meters above sea level from April $26^{\text {th }}, 2014$ to January 26, 2015. In the spring, when fern croziers first emerged, we tagged 4 fronds on 
20 plants of Polystichum munitum (Kaulf.) C.Presl (western swordfern) along two transects, each $100 \mathrm{~m}$ long and separated by $50 \mathrm{~m}$. No two sample plants were touching and at least $3 \mathrm{~m}$ apart. Sampling was conducted approximately every 30 days for 10 months total.

Sampling protocol, surface sterilization, and culturing-

At the time of establishment of the study site, one leaflet (i.e. pinna; the unit of sampling) per frond ( $\mathrm{N}=80$ per month) was isolated with fine-tipped gardening shears, immersed in ethanol and wiped dry with a clean Kimwipe (Kimberly-Clark, TX, USA). Each leaflet was placed in a separate unused coin envelope and transported to the laboratory within 2 hours for refrigeration. Within 24 hours of sampling, leaflets were surface sterilized in sequential baths of $95 \%$ ethanol, $0.5 \%$ sodium hypochlorite, and $70 \%$ ethanol (v/v; 10 sec, $2 \mathrm{~min}, 2 \mathrm{~min}$, respectively; Arnold et al. 2003) in a sterile hood and allowed to dry on a fresh Kimwipe. One hundred mg of each leaflet was utilized for DNA extractions and remaining tissue was plated onto malt extract agar (MEA) medium $(2 \% \mathrm{w} / \mathrm{v})$ for culturebased analysis. After a period of 7 days, any fungi emerging from the surface sterilized leaflets were re-isolated onto fresh MEA plates, creating axenic cultures. Culture-independent DNA extraction and library preparationFollowing each monthly sampling event, fresh tissue was homogenized in a bead mill (TissueLyser II; Qiagen, Hilden, Germany) prior to DNA extraction with pre-sterilized tungsten carbide beads according to the manufacturer's instructions. Total DNA was extracted with the Qiagen DNeasy plant mini kit (Qiagen, Hilden, Germany). A negative control was included in each round of DNA extraction and included a pre-sterilized bead. Extracted DNA was stored at $-80^{\circ} \mathrm{C}$ until library preparation. Fungal DNA was 
amplified with the fungal primers ITS1F and ITS2 that also contained Illumina MiSeq adaptor sequences and a $12 \mathrm{bp}$ unique barcode on the reverse primer for each sample (Smith and Peay 2014). Reactions contained $5 \mu \mathrm{L}$ of $5 \mathrm{X}$ Phusion High Fidelity buffer, $0.5 \mu \mathrm{L}$ of $10 \mathrm{mM}$ dNTPs, $0.5 \mu \mathrm{L}$ of each primer at $10 \mu \mathrm{M}, 0.63 \mathrm{U}$ of Phusion High Fidelity polymerase (New England BioLabs, MA, USA), $1 \mu \mathrm{L}$ of template DNA (5-30 $\mathrm{ng} / \mu \mathrm{L}$ ), and diluted up to $25 \mu \mathrm{L}$ with PCR water. Thermocycler conditions were as follows: $94^{\circ} \mathrm{C}$ initial denaturation for $60 \mathrm{sec}$ and 30 cycles of $94^{\circ} \mathrm{C}$ for $30 \mathrm{sec}, 52^{\circ} \mathrm{C}$ for $30 \mathrm{sec}$, and $68^{\circ} \mathrm{C}$ for $30 \mathrm{sec}$ with a final elongation at $68^{\circ} \mathrm{C}$ for 7 minutes. Reactions were held at $4^{\circ} \mathrm{C}$ until removed from the thermocycler and verified with gel electrophoresis. Fungal DNA from each sample was amplified in duplicate, pooled, and cleaned with the Agencourt AMPure XP kit (Beckman Coulter, CA, USA). Final concentrations of cleaned PCR products were verified with the Qubit Fluorometer (Life Technologies, CA, USA) according to the manufacturer's instructions. Ten ng of each cleaned sample was added to the final library, diluted to $10 \mathrm{nM}$ and sequenced on the Illumina MiSeq platform 2 x 250 bp with a 30\% PhiX spike added to the sequencing run. Each sequencing run (5 total) comprised two months of samples from the same 20 plants ( $n=160)$, extraction controls from each month $(n=2)$, pooled PCR negative controls ( $n$ $=1$ ), and a positive control comprised 30 different fungal taxa from the phyla Basidiomycota and Ascomycota obtained from our region and pooled in equimolar amounts.

Sequence processing and bioinformaticsForward and reverse reads from each sequencing run were merged with USEARCH v10.0.240 (Edgar and Flyvbjerg 2015) and overlapping regions that did not match on the 
forward and reverse reads by at least $90 \%$ were discarded. Forward and reverse primer sequences were removed with Cutadapt v1.10 (Martin 2011) and any reads with an expected error threshold greater than 1.0 were removed (6.2\% of total reads) with USEARCH. Following these sequence filtering steps, 15.3 M sequences remained for the analysis. Sequences were denoised into zero-radius operational taxonomic units (zOTUs; also known as exact sequence variants or ESVs) using the UNOISE3 algorithm implemented in USEARCH (Edgar 2016b). We acknowledge the recent trend of utilizing ESVs in lieu of OTU clustering in NGS amplicon datasets due to increased reproducibility across datasets (Callahan et al. 2017; Thompson et al. 2017); however, the potential exists for grouping two strains that would taxonomically be classified as the same species into separate ESVs due to minor intergenomic differences in the sequenced regions, thereby inflating richness estimates (Glassman and Martiny 2018). For comparison, we verified the number of OTUs generated through clustering at 97\% sequence similarity with the UPARSE algorithm (Edgar 2013) and found a total of 1292 OTUs (versus $985 \mathrm{ESVs}$ ). Therefore, we are confident that the denoising process employed in this dataset did not artificially inflate richness and likely resulted in a more accurate representation of the actual taxa sequenced from the study site. For the sake of simplicity, we will hereafter refer to ESVs as OTUs in this study owing to the utility and widespread use of the latter initialism. Taxonomic assignments were made through the SINTAX algorithm (Edgar 2016a) using the most recent version of the UNITE ITS database (v01.12.2017; Kõljalg et al. 2013) and added to the OTU matrix. Control samples were removed from the final OTU matrix by subtracting the total number of reads of all controls from the actual samples (Nguyen et al. 2014; Younginger and 
Ballhorn 2017). The resultant OTU matrix and associated metadata were imported into the R v3.3.2 (R core team 2016) packages phyloseq v1.19.1 (McMurdie and Holmes 2014) and vegan v2.4.2 (Oksanen et al., 2011) for statistical analyses (below) and figure generation with ggplot2 v2.2.1 (Wickham 2009). The culture-independent component of this study revealed a dominant endophyte that rapidly colonized all host plants at the study site (OTU1) and an isolate of this taxon was further examined in in vitro competition assays and gametophyte assays (below).

$\underline{\text { In vitro }}$ competition assays-

DNA from the aforementioned axenic cultures of fungi, generated from the same host plants, was extracted with the REDExtract-N-Amp kit (Sigma-Aldrich, MO, USA) following the manufacturer's instructions and amplified utilizing the primers ITS1F and ITS4 (White et al. 1990). PCR reactions contained 12.5 $\mu \mathrm{L}$ of 10X GoTaq PCR Master Mix (Promega, WI, USA), $1.25 \mu \mathrm{L}$ of $10 \mathrm{mg} / \mathrm{mL}$ BSA, $1.25 \mu \mathrm{L}$ of each primer at $10 \mu \mathrm{M}$, $1 \mu \mathrm{L}$ of template DNA, and diluted up to $25 \mu \mathrm{L}$ with PCR water. Thermocycler conditions were as follows: $94^{\circ} \mathrm{C}$ initial denaturation for 3 min and 35 cycles of $94^{\circ} \mathrm{C}$ for $1 \mathrm{~min}, 50^{\circ} \mathrm{C}$ for $1 \mathrm{~min}, 72^{\circ} \mathrm{C}$ for $1 \mathrm{~min}$ with a final elongation at $72^{\circ} \mathrm{C}$ for 10 minutes. Reactions were held at $4^{\circ} \mathrm{C}$ until removed from the thermocycler and verified with gel electrophoresis. Extracted DNA was submitted for Sanger sequencing (Functional Biosciences, WI, USA). Raw sequences were removed of forward and reverse primers, concatenated through Geneious v10.0.5 (Kearse et al. 2012), and exported to a single fasta file. Representative OTU sequences from the NGS dataset were then blasted against compiled sequences from the cultures through the BLASTn algorithm v2.2.28 (Camacho et al. 2009). The output was sorted by percent sequence 
similarity and then by the length of the match. Any sequences that did not possess $100 \%$ sequence similarity over at least $150 \mathrm{bp}$ of the NGS read were discarded. Any cultures that possessed a $100 \%$ sequence match to a given OTU were then grouped together and compared by morphology and sequence similarity in Geneious. We then performed alignments in Geneious with the MAFFT v1.3.7 algorithm on sequences from the NGS fasta file and the fasta file from cultures for the following reasons: a given NGS OTU may have matched multiple cultures in our collection, many of the cultures were likely of the same species, and some of the cultures had the same taxonomic assignment, particularly when unable to resolve to species. Through this process, we confirmed that any duplicate cultures that matched a particular OTU in the NGS dataset were indeed the same species (or at least 100\% similar to each other over the entire ITS region and $100 \%$ similar to the OTU over $150 \mathrm{bp}$ or greater) with perfect fidelity. A total of 11 of these isolates were then transferred to fresh MEA medium to generate starting material for in vitro competition assays.

Cultures were competed against the dominant OTU from the culture-independent observational study by placing a single punch of equal diameter $(6 \mathrm{~mm})$ with a sterile straw into fresh plates with lignocellulose agar $(2 \% \mathrm{w} / \mathrm{v}$, adjusted to $\mathrm{pH} 5.5$; Sharma and Pandey 2010) at an equal distance from the edge of the plate. A new media source was chosen for the assays to mimic colonization of a new host and due to the most favorable growth of the dominant OTU1 in preliminary trials. Plates were sealed and placed in a cardboard box for one month. After one month, plates were removed and photographed on a table with $3 \mathrm{~cm}$ gridlines for analysis with a macro lens on a digital single-lens reflex camera (Canon, Tokyo, Japan). Plates were then scored by calculating the 
difference in growth of the competitor versus the growth of OTU1 in the direction towards each other (Wardle and Parkinson 1992; Schwelm et al. 2009). All measurements were conducted with the FIJI version of ImageJ software v2.0.0 (Schindelin et al. 2012; Rueden et al. 2017), results were analyzed in R and figures were generated with ggplot2.

Gametophyte assaysSpore-bearing leaves of P. munitum were collected from the same plants after the NGS observational study was complete on 9/18/17. Leaves were dried in a closed container with silica gel. After two weeks of drying, leaves were scraped of sori and collected in a sterile falcon tube. Ten mg aliquots of these spores were then placed in sterile $2 \mathrm{~mL}$ Eppendorf tubes and soaked overnight in autoclaved $\mathrm{DDH}_{2} \mathrm{O}$ on a shaker rotating at 40 $\mathrm{rpm}$. Spores were spun at $2000 \mathrm{rpm}$ for 3 minutes and the supernatant was decanted. Spores were sterilized by immersion in a solution of $5.5 \% \mathrm{NaOCl}$ and $0.1 \%$ Tween 20 (v/v) and vortexed for 10 minutes (Fernandez et al. 1993). Samples were rinsed three times with PCR water with a 2000 rpm centrifuge step ( $3 \mathrm{~min})$ between each rinse. Spores were then washed with $70 \% \mathrm{EtOH}(\mathrm{v} / \mathrm{v})$ for $30 \mathrm{sec}$ and rinsed with PCR water and centrifuged twice more. Spores were re-suspended in PCR water and diluted to 3500 spores $\mathrm{mL}^{-1}$ with a Double Neubauer Counting Chamber. Fifty $\mathrm{mL}$ of the spore suspension were plated onto Modified Parker/Thompson's Basal Nutrient Medium (Klekowski 1969) and culture dishes were placed near a window with indirect sunlight for a period of two months and were visually inspected with a stereo microscope (Leica, IL, USA) for visible contamination prior to fungal treatments. 
Suspensions of fungal conidia were created from the dominant endophyte detected through culture-independent methods and isolated from the same plants and study site in culture-based work (OTU1). Additionally, another culture of Plectania milleri (also isolated from the same plants) served as a source of fungal inoculum. Suspensions were generated by flooding culture dishes with $10 \mathrm{~mL}$ of $0.05 \%$ Tween 80 and hyphae were carefully scraped with autoclaved microscope slides twice. This material was poured into a $15 \mathrm{~mL}$ sterile falcon tube packed with autoclaved cotton balls, creating a filter for fungal fragments. Once the suspension was filtered, cotton was removed with sterile forceps and falcon tubes were spun in a centrifuge at $2000 \mathrm{rpm}$ for five minutes. The supernatant was discarded, and suspensions were rinsed with sterile $\mathrm{DDH}_{2} \mathrm{O}$ and centrifuged twice more. Conidia were resuspended in $10 \mathrm{~mL}$ of sterile $\mathrm{DDH}_{2} \mathrm{O}$ and diluted to $1 \times 10^{5}$ cells $\mathrm{mL}^{-1}$ with a Double Neubauer Counting Chamber. One hundred $\mu \mathrm{L}$ of the respective suspensions (OTU1 and Plectania milleri) were pipetted onto treatment groups (ca. $1 \times 10^{4}$ conidia) and $100 \mu \mathrm{L}$ of sterile $\mathrm{DDH}_{2} \mathrm{O}$ was pipetted onto controls $(\mathrm{N}=30)$. Changes in surface area were recorded after a period of 0,4 and 8 weeks by generating images with a digital camera as described above (Culturebased Competition Assays). Surface area $\left(\mathrm{cm}^{2}\right)$ was analyzed in ImageJ with a custom macro which converted images to RGB, and utilizing the blue image only, thresholding to highlight only gametophytic tissue. Thresholding was manually adjusted to ensure consistent capture of tissue if necessary. Surface area measurements were exported and concatenated with a custom python script. Results were analyzed in R and figures were generated with ggplot2. At the conclusion of the experiment, total DNA was extracted from each gametophyte and amplified with the primers and PCR reaction conditions 
described in in vitro competition assays above. PCR was verified with gel electrophoresis and submitted for Sanger sequencing. Resultant sequencing reads were aligned in Geneious and compared to sequences generated from source cultures. All treatments were confirmed to be axenic with the appropriate fungal strain and control gametophytes yielded no fungal sequences.

Statistics-

Samples were grouped by plant in R (4 samples per plant within each month) prior to any statistical analyses as pooling samples computationally (versus physically) has been shown to result in greater richness recovered from sampling efforts (Song et al. 2015). Following pooling, species accumulation curves were constructed by finding the mean OTU richness from the culture-independent OTU matrix following 999 permutations with a first order jackknife method of estimating the extrapolated number of OTUs (Chiarucci et al. 2008; Colwell et al. 2012). Following curve construction, the matrix was rarefied by randomly sampling with replacement to 2800 sequences per sample. This resulted in the loss of one sample (plant 1 in April) and 193 OTUs, which was an acceptable compromise for more robust conclusions of community dynamics. Alpha diversity metrics were calculated with Hill numbers at the scales of $q=0,1$, and 2 (Hill 1973), where q represents the exponent of each Hill number. As the value of q increases, the measure becomes less sensitive to rare taxa, placing more emphasis on common taxa. A value of $q=0$ represents OTU richness, $q=1$ represents the exponential of Shannon entropy, and $\mathrm{q}=2$ represents the inverse of the Simpson index (Chiu and Chao 2016). Differences in each richness measure between sampling months were examined with repeated-measures ANOVA by specifying plant individuals as an error stratum, thereby 
removing the variance attributed to plant individuals in the final model. An ordination plot of community composition, grouped by plants within each sampling month, was generated through non-metric multidimensional scaling (NMDS) with a square root transformation and Wisconsin double standardization following the generation of a BrayCurtis dissimilarity matrix. Significant differences between sampling months were examined with a repeated-measures permutational multivariate analysis of variance (PERMANOVA; Anderson 2001, Anderson and Walsh 2017) following 999 permutations with Bray-Curtis dissimilarity while again treating plant individuals as an error stratum. Additionally, an examination of significant differences between the dispersion of group centroids (corresponding to sampling month), visualized in ordination, was determined through a multivariate version of Levene's test for variance homogeneity (Anderson 2006). Following ordination, sequencing read numbers were converted to relative abundance and any taxa that were represented in less than $5 \times 10^{-5}$ of the total dataset were removed for easier visualization in the taxonomy plot. Finally, differences in gametophyte surface area (from gametophyte assays) were determined between weeks 0 and 4 and weeks 0 and 8 for each treatment and tested with one-way ANOVA following an examination of data distributions to fulfill parametric assumptions. Results Rarefaction curves and alpha diversity estimatesFor the culture-independent study, we observed a total richness of 985 OTUs across all sampling months. Species accumulation curves reveal a sharp increase in richness from April (when foliar tissue first emerges) to May (Figure 2.1). This richness begins to decline in June and dramatically falls in July, at which point there is substantial overlap 
in $95 \%$ confidence intervals for the remaining sampling months with the exception of August and September, which exhibit even lower richness. After rarefying the OTU matrix to an even sampling depth, 792 total OTUs remained in the dataset. When host leaves first emerged in April, 162 OTUs were observed across all of the plants sampled. Richness at the study site rapidly increased after just 30 days to 582 OTUs in May, then decreased again in June to 229 OTUs. For the remaining sampling months (July through January), the richness at the study site sharply declined to 17 OTUs in August and increased slowly to 64 OTUs by the end of the sampling period. This decline in total richness was accompanied by a concomitant increase in the number of samples which contained reads from the dominant taxon, OTU1. A further examination of alpha diversity through Hill numbers (Bálint et al. 2015; Chiu and Chao 2016; Younginger and Ballhorn 2017) demonstrated the greatest median richness in May ( $q=0$; Figure 2.2). When $\mathrm{q}=1$ (exponential of Shannon entropy), the greatest diversity observed across sampling months occurred in May; beyond this sampling month, diversity remained close to one (June through January). When $\mathrm{q}=2$ (inverse of Simpson index), the most emphasis is placed on abundant taxa; here we found more diversity in April than in May, but again the remaining sampling months had an effective diversity of 1 due to the presence of OTU1. 


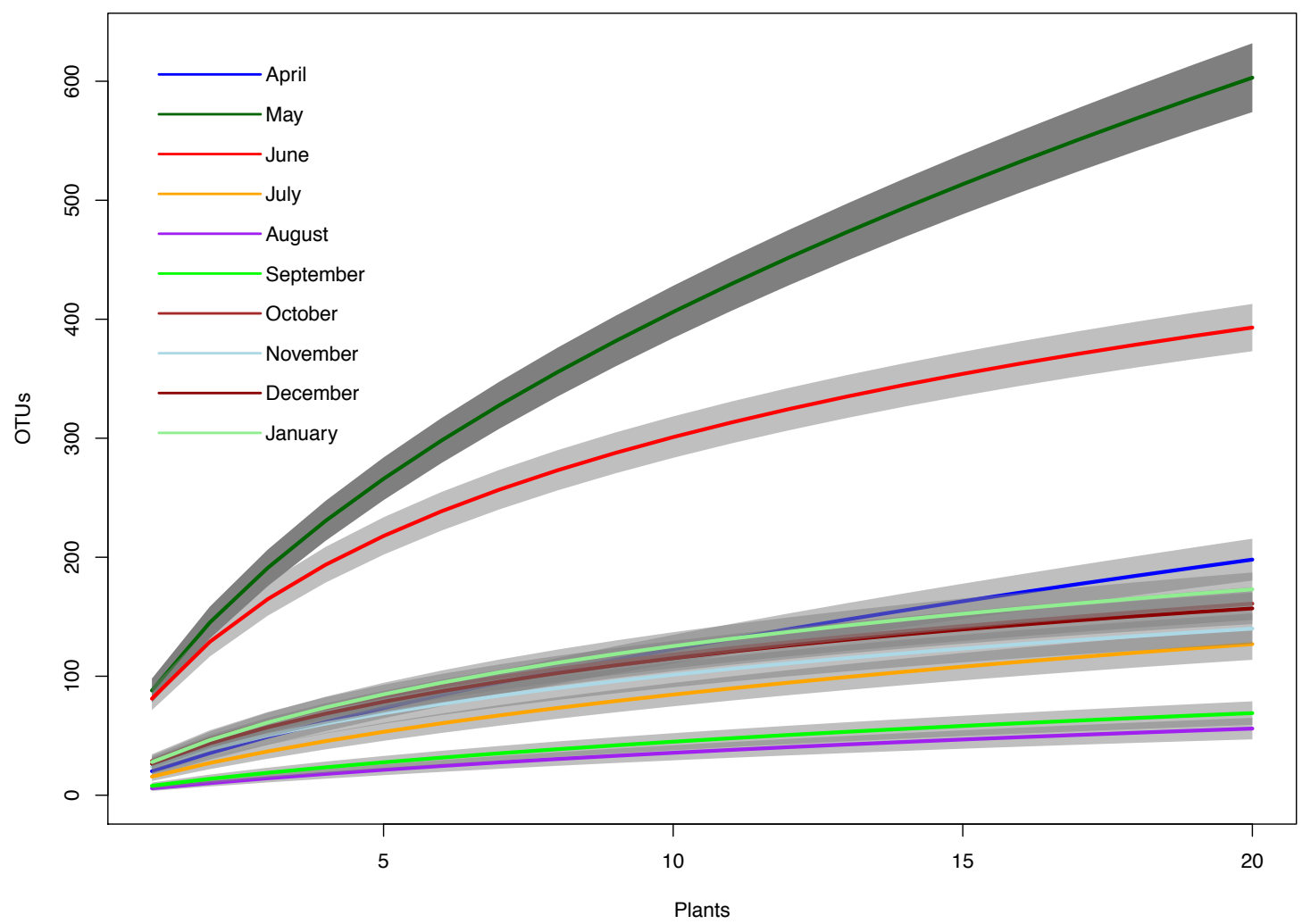

Figure 2.1 Species accumulation curves of operational taxonomic unit (OTU) richness of each sampling month in the culture-independent study. Curves represent mean OTU richness following 999 permutations and shaded regions represent $95 \%$ confidence intervals $(\mathrm{N}=20$ plants per month). 


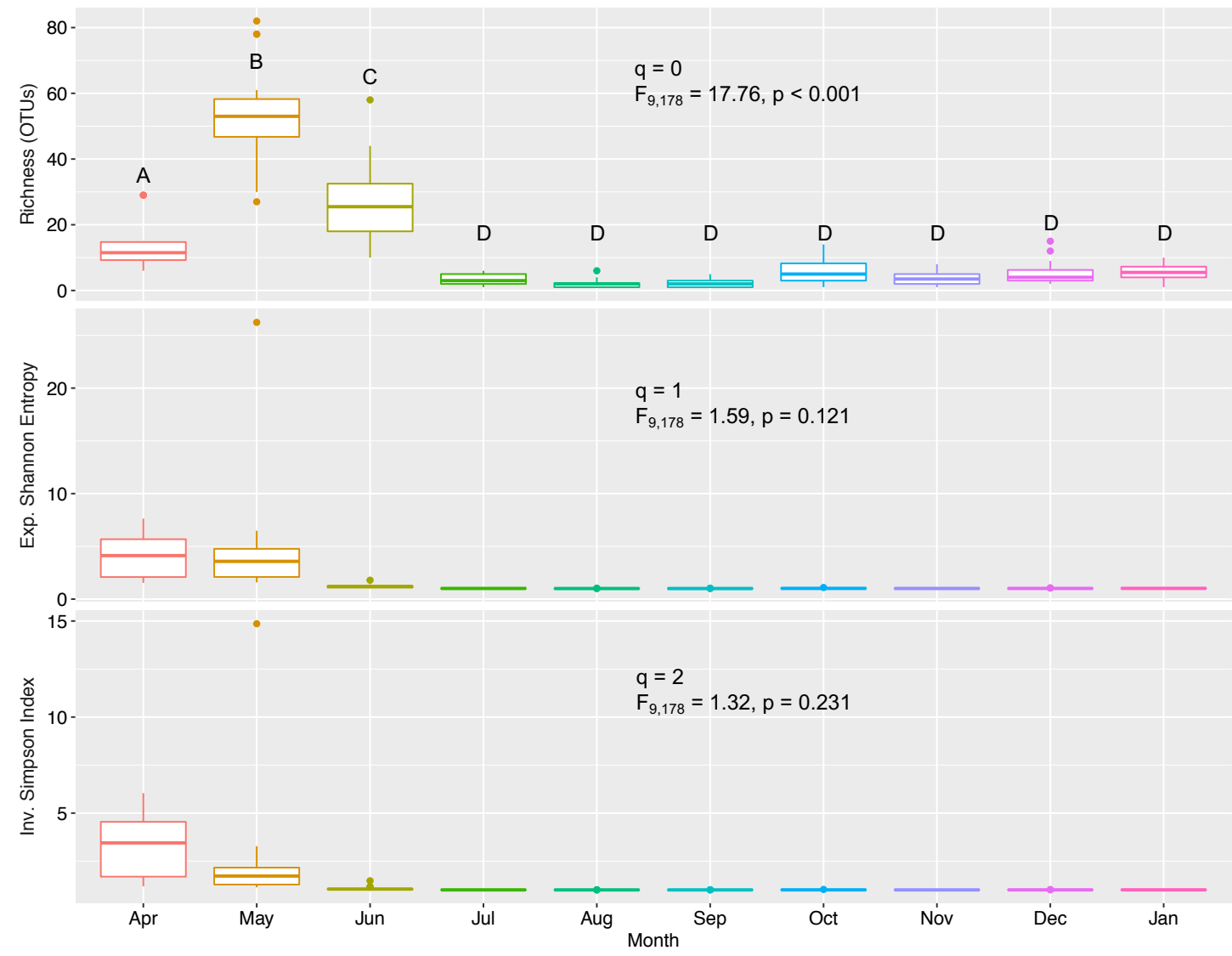

Figure 2.2 Hill measures of alpha diversity at the scales of $q=0,1$, and 2 of each sampling month in the culture-independent study. As the value of q increases, the alpha diversity measure becomes less sensitive to rare taxa. Differences in Hill measures were examined with repeated-measures ANOVA and Tukey's HSD post-hoc analysis. Boxplots represent the median value (middle line) for each plant within each month, boxes represent upper and lower quartiles and lines represent upper and lower deciles $(\mathrm{N}=20$ plants per month).

Beta diversity and ordination-

Ordination through NMDS reveals a highly heterogeneous grouping of endophyte

communities in host plants and a significant difference in community composition over

the course of the growing season (PERMANOVA: Pseudo- $\mathrm{F}_{9,188}=24.88, \mathrm{p}<0.001$;

Figure 2.3). Ninety-five percent confidence ellipses show significant differences in dispersion about centroids between sampling months (PERMDISP2; Pseudo- $\mathrm{F}_{9,188}=$ 118.12, $\mathrm{p}<0.001)$ with no overlap in April with other sampling months in the dataset. In May, a compression in dissimilarity is observed between host plants as endophytes 
become more evenly distributed across the study site. In June, a slight overlap in confidence ellipses is seen with May, yet the communities appear distinct from other sampling months. For the remaining sampling months (July - January), there is much overlap of community composition between plants within a given sampling month and nearly complete overlap between sampling months. This result is driven by the presence of OTU1 and the exclusion of other taxa. Overall, the NMDS plot shows a high degree of beta diversity in the first three months of the growing season, with very little beta diversity between the remaining months.

Taxonomy-

In newly emerged fern leaves examined in the culture-independent study, the endophyte community is colonized most abundantly by taxa from three fungal classes: the Agaricomycetes, Leotiomycetes and Sordariomycetes (Figure 2.4). After one month of exposure in the field, the foliar tissue of the host is rapidly colonized by OTU1 (assigned to Geomyces Traaen within the Leotiomycetes). All other taxa decline in relative abundance through time, yet OTU1 comprises nearly $100 \%$ of sequencing reads from July - January. 


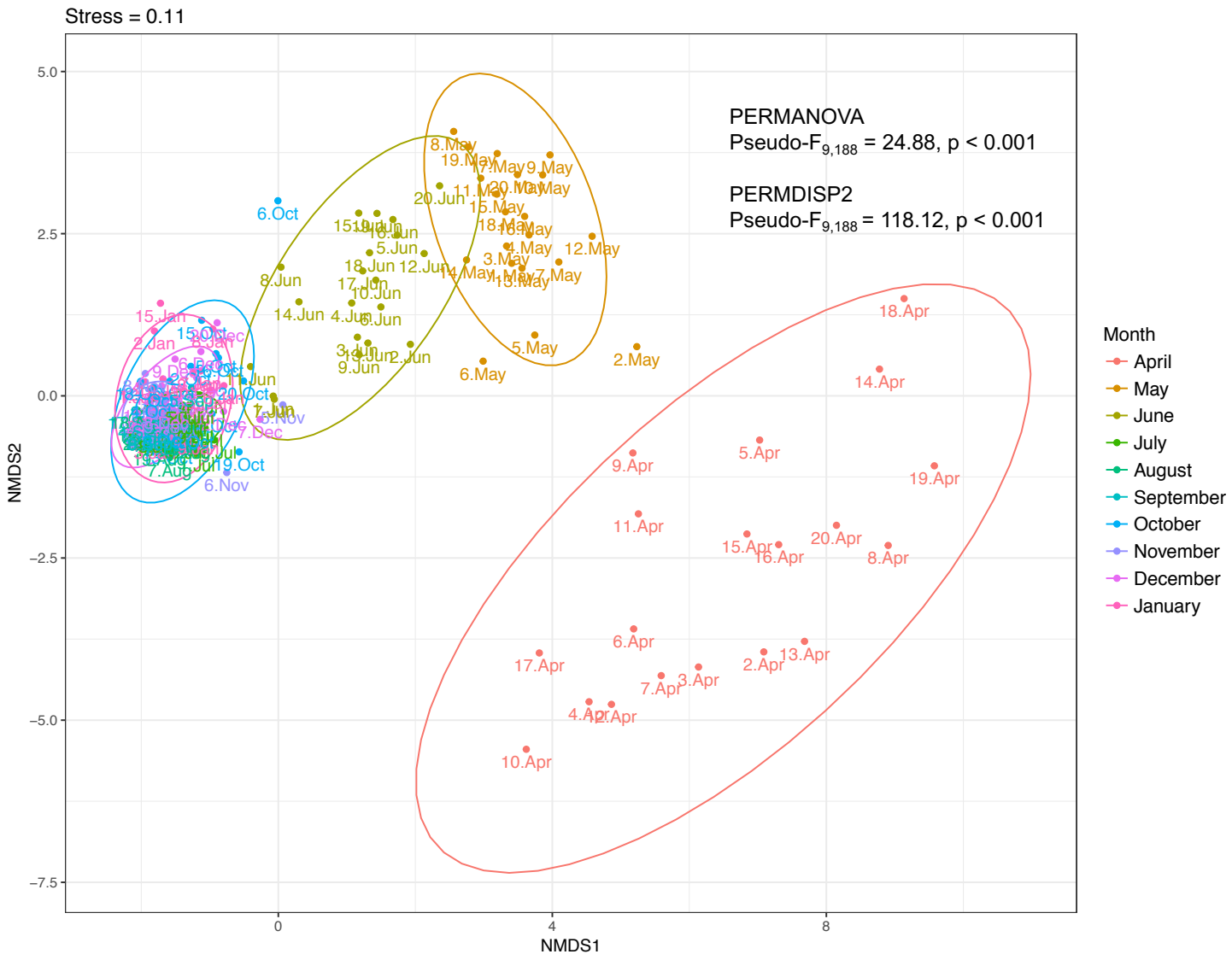

Figure 2.3 Non-metric multidimensional scaling (NMDS) of endophyte community composition within each sampling month in the culture-independent study. Values were square root transformed and Wisconsin double standardized following the generation of a Bray-Curtis dissimilarity matrix from the OTU table. Points represent each plant at the study site, colored by the sampling month and ellipses represent $95 \%$ confidence intervals. Stress is equal to 0.11 . Differences in community composition throughout the sampling period were examined with a repeated-measures permutational multivariate analysis of variance (PERMANOVA) following 999 permutations of Bray-Curtis dissimilarity while holding plant individuals as an error stratum. Significant differences in group dispersion about each centroid were tested with a multivariate version of Levene's test for variance homogeneity (PERMDISP2; $\mathrm{N}=20$ plants per month). 


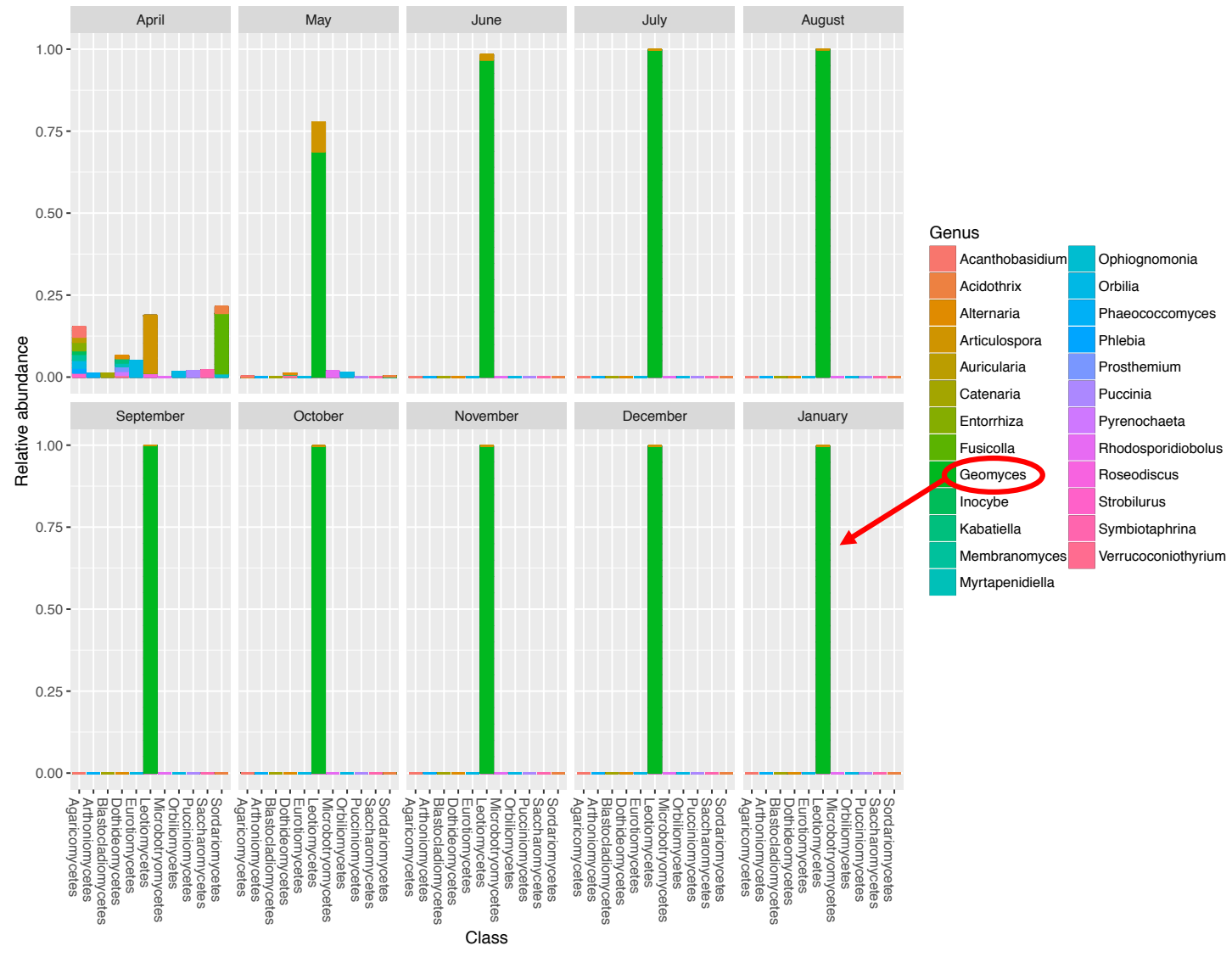

Figure 2.4 Taxonomy plot of endophyte community composition within each sampling month of the culture-independent study. Colored bars represent the relative abundance of genera found within each fungal class (x-axis). Any taxa that represent less than $5 \times 10^{-5}$ of the total dataset were removed for easier visualization $(\mathrm{N}=20$ plants per month). 


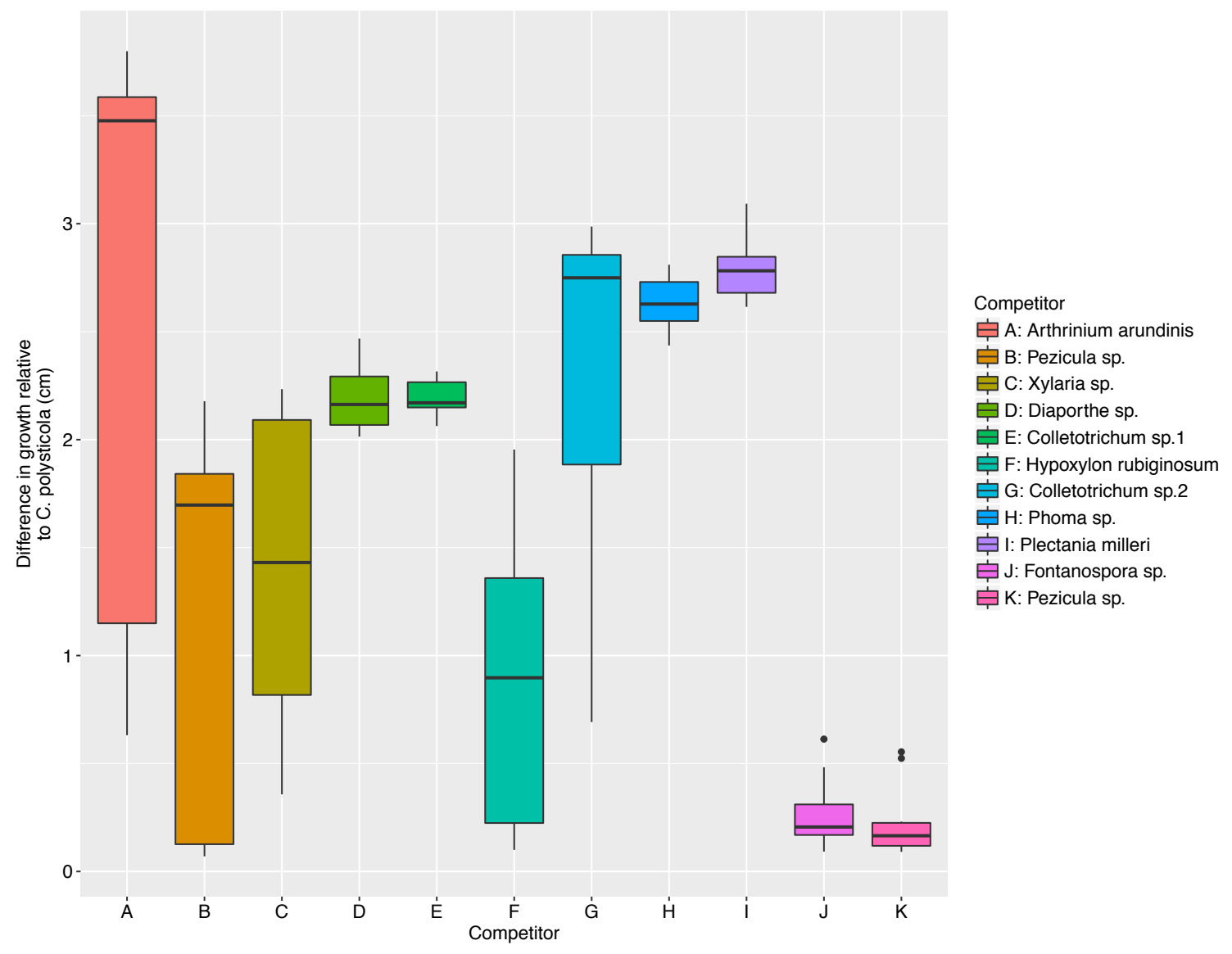

Figure 2.5 Results of in vitro competition assays of OTU1 versus 11 other strains recovered from the same plants examined in the culture-independent study. Boxes represent the difference in growth of each competitor relative to the growth of OTU1 in the direction towards one another in the culture dish. Boxplots represent the median value (middle line) for each pairwise comparison, boxes represent upper and lower quartiles and lines represent upper and lower deciles $(n=12$ replicates per competitor; 132 plates total).

In vitro competition assays-

All of the 11 taxa grown in culture with OTU1 demonstrated greater growth rates over one month (Figure 2.5). In fact, 5 of the 11 taxa tested exhibited a two-fold greater growth rate than OTU1 on LCA medium. These results were not restricted to LCA, as preliminary trials were conducted between OTU1 and several taxa on MEA, potato dextrose agar (PDA), Reasoner's 2A agar (R2A), Rose Bengal agar and a medium made from $4 \mathrm{~g}$ of lyophilized and ground P. munitum tissue with $20 \mathrm{~g}$ of agar $\mathrm{L}^{-1}(2 \% \mathrm{w} / \mathrm{v}$; fern medium), all confirming that OTU1 performs poorly in competition assays owing to slow 


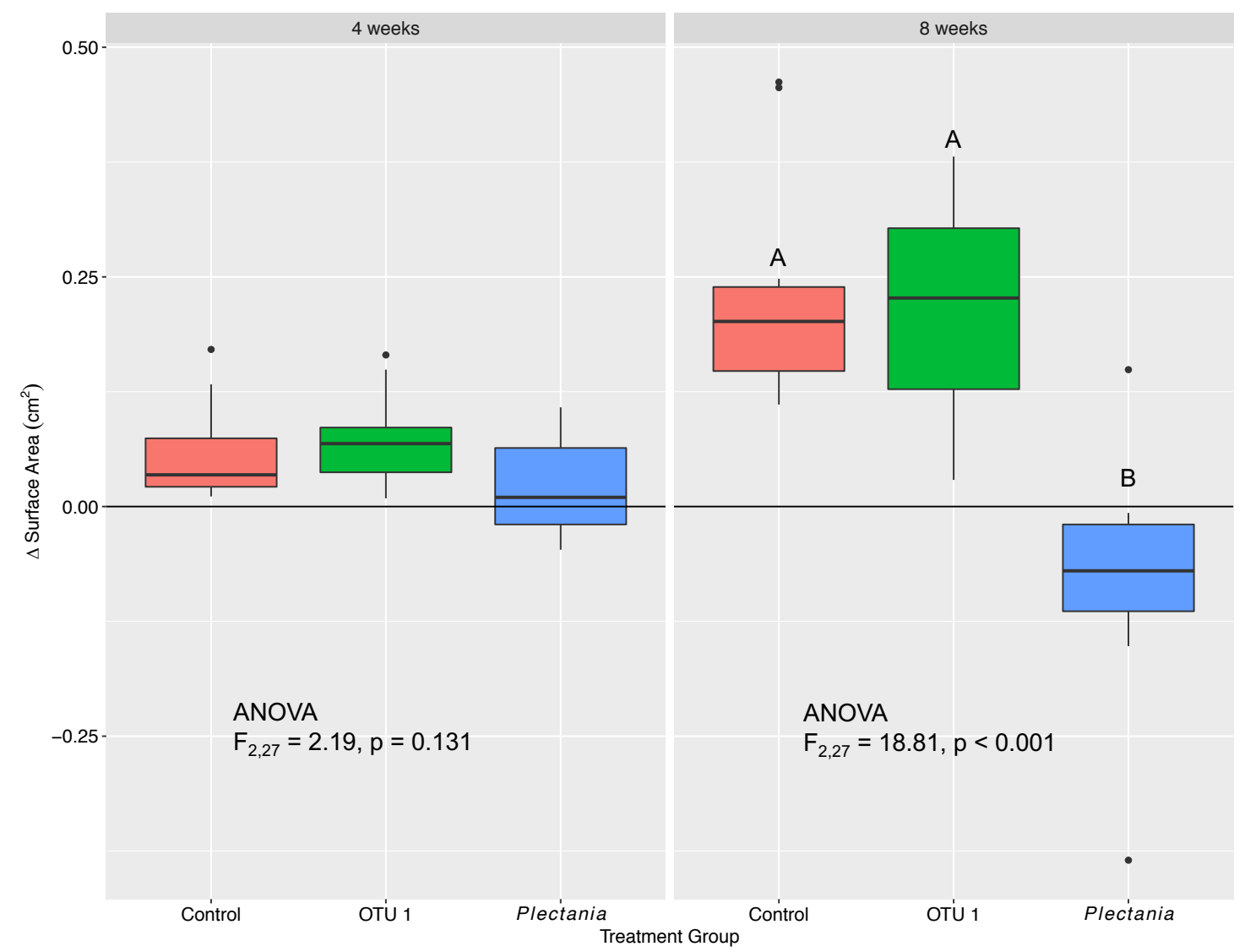

Figure 2.6 The change in surface area $\left(\mathrm{cm}^{2}\right)$ of $P$. munitum gametophytes treated with conidia suspensions generated from fungal cultures initially isolated from the host plants examined in the culture-independent study (OTU1 and Plectania milleri) or with sterile $\mathrm{DDH}_{2} \mathrm{O}$ (control). Spores from the fern host were harvested, surface sterilized and plated on Modified Parker/Thompson's Basal Nutrient Medium and allow to grow for two months in indirect sunlight prior to treatments. Images were taken at 0, 4 and 8 weeks after inoculation and surface area was analyzed with ImageJ through a custom macro. Differences in surface area were determined by subtracting the surface area at week 0 from week 4 and also at week 8 . Differences were examined with one-way ANOVA at 4 and 8 weeks and further analyzed with Tukey's post-hoc HSD. Boxplots represent the median change in surface area (middle line) for treatment groups at 4 and 8 weeks, boxes represent upper and lower quartiles and lines represent upper and lower deciles $(\mathrm{N}=30)$.

growth rates in vitro. In fact, even over an entire year of competitive conditions, OTU1 remains covered by the hyphae of every competitor that it was tested against.

\section{Gametophyte assays-}

At four weeks, OTU1-inoculated gametophytes of $P$. munitum show no difference in surface area $\left(\mathrm{cm}^{2}\right)$ when compared to sterile water controls, while gametophytes inoculated with $P$. milleri show a smaller increase relative to controls, though these 
results are not significant (one-way ANOVA: $\mathrm{F}_{2,27}=2.19, \mathrm{p}=0.131$; Figures $2.6 \& 2.7$ ). At eight weeks, a greater increase in surface area is again observed in OTU1-inoculated gametophytes relative to controls with more variability seen in both groups of plants overall, but this result is not significant. In contrast, gametophytes inoculated with $P$. milleri show a significant reduction in surface area over 8 weeks to below baseline values $\left.\mathrm{F}_{2,27}=18.81, \mathrm{p}<0.001\right)$

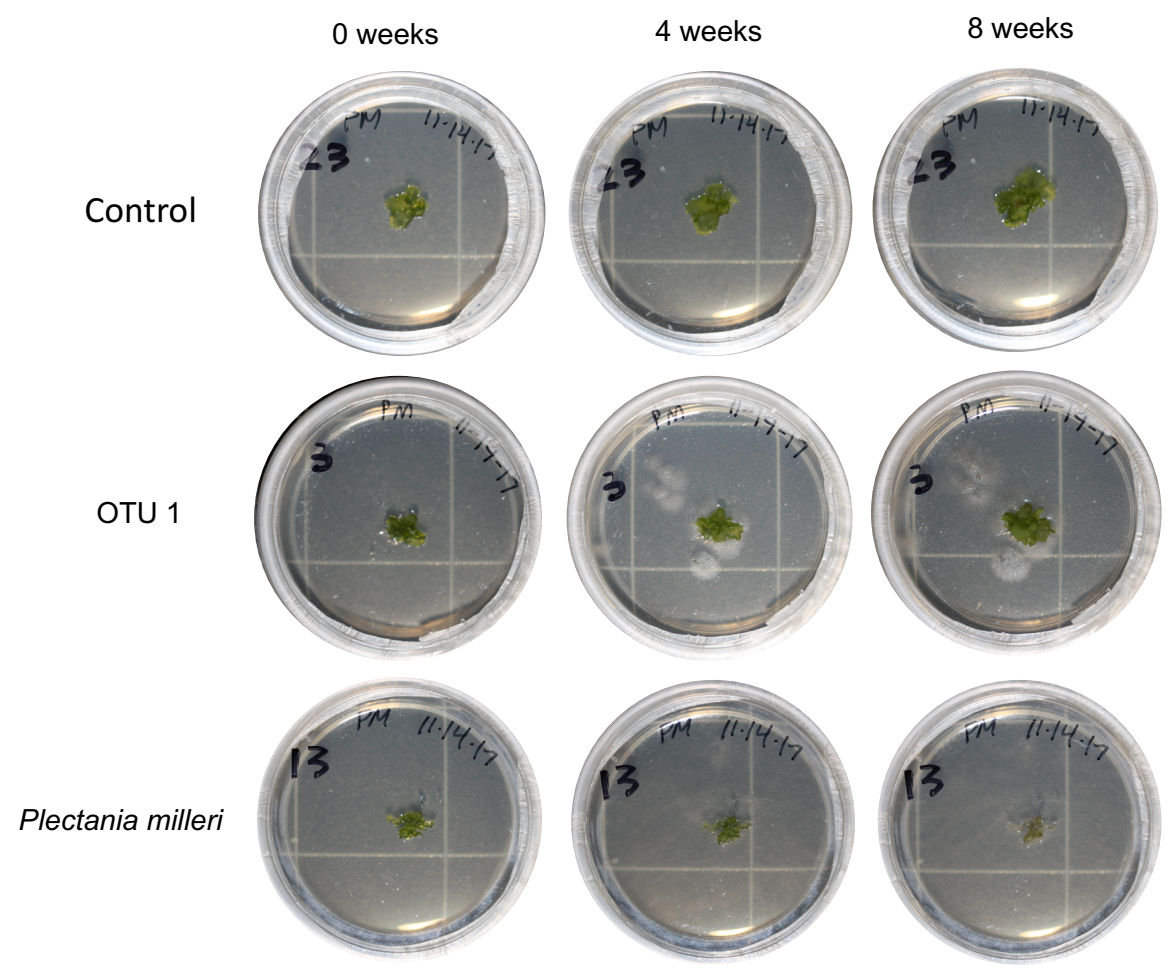

Figure 2.7 Representative images of gametophyte assays. Columns correspond to weeks post-inoculation and rows represent treatment groups.

\section{Discussion}

This study clearly demonstrates the highly dynamic nature of endophyte communities and draws an important distinction between endophytes in ferns and other plant systems. The sampling regime employed on a monthly basis for an entire growing season enables us to make a strong recommendation for the careful timing of observational studies of 
microbial communities. A reliance on sampling efforts at a single point in time could lead to widely varying inferences depending on when these efforts are conducted. Therefore, we suggest the practice of repeated sampling through time to account for the highly dynamic nature of endophyte communities. Further, the culture-independent study coupled with culture-based competition and gametophyte assays has revealed a notable fungal taxon that may play an important ecological role in the host population. Priority effects in this community appear relatively insignificant in early stages of colonization. However, when OTU1 arrives and quickly spreads throughout the host population, it is either able to outcompete neighboring taxa through repeated colonization and competitive exclusion (which was not confirmed from the in vitro results) or its spread is somehow facilitated by the host plant. Results from the culture-independent study contrast sharply with results from other seed plant (Jumpponen and Jones 2010; Peñuelas et al. 2012; Peršoh 2013) and bryophyte systems (Davey et al. 2012). It is clear that endophytes that colonize this temperate fern population obey different community dynamics than would be expected through time (Peršoh 2015). Specifically, as foliar tissue is exposed to fungal inoculum, richness should increase over time until an equilibrium is reached and competitive coexistence prevails (Arnold and Herre 2003; Jumpponen and Jones 2010). In contrast, the observational component of this study demonstrates that although the richness of fungal OTUs initially increases within the fern host early in the growing season, after two months, richness rapidly declines until there is effectively one dominant OTU found at the study site: OTU1. While early colonization by fungal taxa is observed in newly-emerged fern pinnae, just $2-3$ days old, there is far more heterogeneity between plants which is likely the result of patchy colonization. An increase in richness is 
observed one month after foliar tissue has emerged, as presumed, but the colonization of OTU1 first becomes prevalent in May and leads to decreasing richness at the study site in the remaining sampling months. By July, OTU1 comprises most of the sequencing reads and this trend continues throughout the remainder of the growing season. Although sequencing read numbers may be a poor estimate of actual abundance due to PCR and sequencing biases (Amend et al. 2010; Nguyen et al. 2014), the incidence of OTU1 across all plants and sampling months after April likely reflects the sharp temporal turnover and competitive exclusion occurring at the study site.

A closer examination of the temporal turnover of endophyte community composition documents several intriguing trends. First, a significant difference is found in community composition through time. This basic result is unsurprising, yet a deeper look at what is driving this significant difference reveals a distinctive pattern that can be partitioned into two components: community composition between months and composition between plants. When comparing months, the total community at the site in April is dissimilar from all other months throughout the season and grows progressively more similar between months as a result of the increase in OTU1 and a concomitant decrease in all other taxa. Between plants, early colonizing taxa are highly patchy in newly-emerged tissue (in April) resulting in heterogeneous communities on a small scale but become less heterogeneous in May and June and extremely homogenous throughout the remainder of the growing season in a pattern indicative of microsuccession. Therefore, we can summarily state that the early fern endophyte community is very rich and uneven, potentially resulting from stochastic colonization and dispersal limitation, and this rapidly changes to a highly homogenous community between plants and through 
time. From a statistical perspective, a difference in group dispersions in ordination certainly plays a role in the interpretation that community composition changes through time. Additionally, the fact that no overlap is observed in $95 \%$ confidence intervals in April with all other sampling months, and little overlap is seen in the months of May and June when compared to other months leads us to conclude that both community composition and heterogeneity between plants are responsible for the highly significant temporal turnover observed. It is currently unclear if the sharp reduction in richness is found in other populations of $P$. munitum or in other fern species, as no other culture independent studies have examined fern endophyte communities. Additionally, there is no other culture-independent work, to the best of our knowledge, examining the temporal turnover of fungal endophytes monthly, over an entire growing season in any plant host species, making generalizations impossible at this stage of our knowledge.

Taxonomic assignments of NGS sequencing reads show a higher representation of fungi from Basidiomycota in April than expected, as it is currently presumed that most symbiotrophic endophytes belong to the phylum Ascomycota (Higgins et al. 2007; Arnold et al. 2009). Early in the growing season, we observe a relatively even representation of fungal endophytes from the Agaricomycetes of the phylum Basidiomycota which includes mushroom-forming saprotrophs and ectomycorrhizal fungi (Hibbett et al. 2007) - our dataset includes endophytes from the Sebacinaceae and Geastrales family and order, respectively, that are predominantly saprotrophs (Wells and Oberwinkler 1982, Hosaka et al. 2006), the Leotiomycetes of phylum Ascomycota; the most diverse phylum commonly known as sac fungi - our dataset includes Geomyces of Pseudeurotiaceae, a genus commonly associated with animal pathogens and the best 
match to OTU1 (Lorch et al. 2011), Articulospora, an aquatic hyphomycete (Baschien et al. 2013), and another member of Pseudeurotiaceae that was not resolved to genus, but is closely related to OTU1. We additionally recovered sequencing reads early in the growing season from the Sordariomycetes of phylum Ascomycota; including Fusicolla aquaeductuum of Nectriaceae; a plant pathogen (Gräfenhan et al. 2011). Although initially surprised to find taxonomic assignments from such a diverse range of fungi with far different trophic modes than endophytism, the short sequencing reads of NGS technology are known to be better suited for community-based inferences than taxonomic assignments (Peay 2014). Still, even at more inclusive taxonomic rankings that are likely more accurate, the possibility of fungal fruiting events occurring in the spring could have led to the deposition of fungal taxa on the surface of leaves which we detected through sequencing; however, we are confident that our sterilization protocol is robust and the results observed early in the growing season are indicative of ephemeral taxa exploiting an uncolonized habitat (i.e. newly emerged leaf tissue). The sharp temporal turnover that happens in just 30 days (from April to May), results in a reduction of endophytes from the Agaricomycetes and Sordariomycetes, with an increase in representation from the Leotiomycetes. These taxonomic assignments more closely align with current observations of most symbiotrophic endophytes belonging to Ascomycota (Rodriguez et al. 2009). Although the generic taxonomic assignment of Geomyces for OTU1 was made with the most recent annotated database from UNITE (December 1, 2017; Koljag), an alignment of the representative sequence for OTU1 matches the ITS1 region of an isolate obtained from the same plants in culture-based work identically over its entire 206 basepair length. This isolate is part of a new family of fungi (Catenosporaceae), along with 
OTU44 and two other taxa isolated from Mount Saint Helens that belong to the Helotiales of the Leotiomycetes (Chapter 4). The fact that we have isolated both OTU1 and OTU44 on several occasions from the same plants at the study site and their sequences match those obtained from culture-based work with $100 \%$ similarity, leads us to conclude that the OTUs examined in the culture-independent study are the same taxa which belong to Catenosporaceae fam. nov.

One potential explanation for the remarkable increase in abundance of OTU1 relative to other endophytes in the culture-independent study is that it is highly competitive once colonizing the host; however, based on the results of the in vitro competition assays, we can only conclude that OTU1 is not highly competitive in culture. We performed the competition assays on lignocellulose agar (LCA) medium since OTU1 was observed to grow most rapidly, but we also examined the growth of OTU1 in isolation and with competitors in 5 other types of media (some of which are optimal for slow-growing fungi; Ferrari et al. 2011). In an effort to mimic conditions within host tissue most-closely, we further generated "fern medium" by making tea bags with lyophilized $P$. munitum tissue and autoclaving with agar (Sarhan et al. 2016), yet OTU1 was still rapidly outcompeted by other isolates obtained from the same study site (data not shown). We hasten to acknowledge that in vitro work does not truly mimic conditions in planta and OTU1 may behave in a more competitive manner in living tissue. We predict that the results of the culture-independent study are most-likely explained by the following hypotheses which are not mutually exclusive: (i) the abundance of senescent host tissue surrounding the plants serves as a reservoir of inoculum that allows for repeated colonization events, (ii) other vectors including rain and canopy throughfall 
allow for colonization (very little herbivory was observed throughout the study making this type of vector unlikely), (iii) the host plant provides a poor habitat for endophytesas ferns are high in antimicrobial tannins (Mehltreter et al. 2010) — and OTU1 is able to exploit this sub-optimal host to its advantage, though we did not examine changes in host foliar chemistry and (iv) the host or other endophytes facilitate the colonization and/or spread of OTU1 through some other unknown mechanism. Although all of the above are possible, we predict that a combination of (i) the senescent material serving as an inoculum source (i.e. storage effects; Kennedy 2010) and (iii) the ability of OTU1 to better exploit the host resource are leading to the observed results. The senescent fern material is the product of growth in previous years ( $2-5$ years old), may play a role in suppressing understory plant competitors and could be a way for OTU1 to re-colonize living tissue as an endophyte. Once the host plant tissue senesces, OTU1 (which is already present as an endophyte) could switch to a saprotrophic lifestyle where it undergoes sexual reproduction, fruiting, and re-colonization of the living host (Song et al. 2017). If this were the case, it would conform to the Foraging Ascomycete hypothesis, which posits that many fungi utilize the endophytic lifestyle as a method to tolerate unfavorable seasons or disperse to distant sites while carrying out a saprobic lifestyle to complete sexual reproduction and re-colonize hosts as an endophyte (Thomas et al. 2016). It is also currently acknowledged that there is remarkable lifestyle lability observed in fungal endophyte lineages, with some taxa existing as latent pathogens (Carroll 1988), aquatic hyphomycetes (Sokolski et al. 2006; Chauvet et al. 2016), or endolichenic fungi (Arnold et al. 2009), making our prediction even more likely. Additionally, since the $P$. munitum host is likely providing a relatively homogenous 
habitat and resource currency, it certainly makes the realization of competitive exclusion by OTU1 possible (Tilman 2007). Isolations of fruiting bodies on senescent material obtained from the same plants are currently underway to determine if this abovementioned hypothesis is supported.

Since a marked increase of a single taxon at the study site - comprised of a healthy and dense population of $P$. munitum — may indicate a mutualistic interaction occurring between the symbiont and host, we sought to elucidate whether OTU1 acts antagonistically or beneficially towards its host in sterile conditions. We chose to examine these potential outcomes in the haploid, gametophytic stage of $P$. munitum since generating an abundance of sterile plants is tractable with relatively straightforward spore sterilization, and also because the recruitment of juvenile ferns in nature requires the gametophytic stage, making the assay ecologically relevant. We hypothesized that if OTU1 is present at the study site in the abundance indicated in both culture-dependent and-independent methods, it is also likely to colonize the gametophytic stage. Based on the results of the experiment, we conclude that OTU1 is, at minimum, a commensal, especially when compared to the effects of $P$. milleri-a saprotroph initially discovered in leaf litter in Idaho (Paden and Tylutki 1969)—which was also isolated from the same fern population. We observed greater variability in OTU1-inoculated plants after 8 weeks, but also observed a greater increase in surface area relative to controls, though this result is not statistically significant. It is possible that if the experiment were run for an additional four weeks, we would have found a significant difference in the change in surface area relative to controls. Still, this would not have been as ecologically-relevant since $P$. munitum gametophytes are likely transitioning to a sporophytic stage in nature 
beyond this time point (Migliaro and Gabriel Y Galán 2012). Additionally, plants treated with $P$. milleri were greatly antagonized and we risked losing an opportunity to confirm our treatment success via sequencing. It should be noted that $P$. milleri has only previously been described as a saprotroph, thus we are unable to clarify whether its antagonistic effects are found in other host species when living endophytically (Paden and Tylutki 1969). However, we chose to also utilize P. milleri conidia in our fungal suspensions due to its frequent isolation from the same host plants at the Coast Range study site.

We extracted total DNA from the gametophytes after 8 weeks to confirm that inoculated plants were axenic and control plants were sterile. Sequencing confirmed that our protocol was robust and all plants contained their respective endophyte and no other fungi, while control plants remained free of fungi. It is possible that the presence of ungerminated conidia from the fungal suspensions led to the conclusion that inoculated plants were colonized; however, we took particular care to gently pluck gametophytes from the culture dish without also removing medium. Furthermore, due to the fact that both sources of inocula were initially isolated as endophytes from the same sporophytic hosts, we suspect that both were capable of colonizing gametophytes, leading to the observed results. The fact that we did not observe a significant difference in the change in surface area of OTU1-inoculated gametophytes, relative to controls, does not mean that we can confidently state that OTU1 is a commensal, as the absence of proof is not the same as proof of the absence (Zapalski 2011). It is important to consider that if OTU1 is able to colonize the gametophytic stage of $P$. munitum in nature, it may enable its host to 
evade pathogenic fungi, thereby altering the evolutionary trajectory of both partners in a highly context-dependent manner.

\section{Conclusions}

This study highlights a striking case of a plant-associated microbial community that runs counter to our current understanding of the temporal turnover of endophytes in nature (Peñuelas et al. 2012). Although it is presumed that endophyte communities should show increased richness and diversity through time, eventually reaching a stable equilibrium of coexistence (Arnold and Herre 2003; Jumpponen and Jones 2010), this appears to be the exact opposite in the host population and future work should address whether this is the case in other fern systems. Based on the evidence collected, we predict that the exclusion of competitors by OTU1 is the result of weak priority effects by the earliest colonists (Vanschoenwinkel et al. 2007; Cline and Zak 2015; Fukami 2015), strong storage effects resulting from fungal inoculum in senescent host tissue (Chesson 2000; Kennedy 2010) and a homogenous habitat created by the host. Were the host to provide a more heterogeneous habitat, competitive coexistence would likely prevail and result in greater richness than observed (Tilman 2007). Additionally, although we demonstrate that OTU1 is an inferior competitor in culture conditions, we predict that it relies upon extensive colonization events from senescent material early in the season and is somehow able to better exploit the habitat created by the host to drive out neighboring endophyte taxa. Finally, we clearly show that OTU1 is at least a commensal on the gametophytic stage of its host, and when considered in the context of abundant fungal pathogens that may antagonize plants in nature, could enable its host to evade pathogens at this vulnerable stage of its life cycle. Taken together, these results clearly show a previously undescribed 
phenomenon occurring in fern microbial communities and further emphasize the importance of relying on repeated sampling efforts before conclusions are drawn about the community composition of these highly dynamic microbes. It is vitally important that we encourage research foci on endophyte communities to move beyond traditional plant systems of economic importance, since exciting and ecologically-relevant endophyte interactions are occurring in other hosts, waiting to be discovered. 


\section{Chapter 3}

Habitat filtering leads to distinct endophyte communities in ferns over a spatial gradient

Introduction

The examination of fungal endophyte community composition is an actively expanding field of research (Vincent et al. 2015; U'Ren and Arnold 2016; Koide et al. 2017), but determining whether these cryptic microbes follow the same tenets of community ecology_initially developed for macroorganisms — remains a challenge (Peršoh 2015). While a major focus of fungal ecology has centered on the study of soil and pathogenic fungi (Benhamou 2004; Peay et al. 2010; Moeller and Peay 2016), fungal endophytes (hereafter endophytes) are unique in their reduced or absent sexuality when colonizing hosts (Thomas et al. 2016), frequent existence as plant commensals (Busby et al. 2015a), formation of highly-localized infections that are transmitted horizontally between hosts (Arnold et al. 2009) and cryptic lifestyle, making differences in their ecology quite likely when compared to other fungi. Additionally, although a strong desire exists to leverage symbiotic microbes in agricultural settings (Gundel et al. 2013; Busby et al. 2017), a clear understanding of the contexts in which habitat filtering (Kivlin et al. 2014) or priority effects (Chase 2003; Fukami 2015) occur is of critical importance. Inoculations with beneficial agents may prove to be a futile effort in highly dynamic microbial communities which may adhere more strongly to naturally-occurring ecological phenomena than to management efforts.

One ecological factor which has been little studied in endophyte communities is the spatial turnover in specific host plants - defined as changes in community 
composition over geographic distance (Ellis et al. 2006; Stegen et al. 2013). Despite a small number of culture-independent studies examining spatial differences in endophyte communities (Zimmerman and Vitousek 2012; Meiser et al. 2014), we still lack predictive framework of ecological principles that may help to clarify why endophyte communities are different through space (if they indeed are). For example, distance decay is an often-cited phenomenon in the literature regarding microbial communities (Hanson et al. 2012; Schmidt et al. 2013; Kivlin et al. 2014). This scenario occurs through a failure of certain taxa to colonize distance sites, resulting in localized speciation, functional redundancy of non-related taxa over larger ecological scales and a greater degree of gamma diversity (Meiser et al. 2014). Though likely to occur through habitat filteringin which varying abiotic factors drive differences in the ability of certain taxa to colonize and persist in a given site - a further challenge for any new microbial colonist is the presence of co-occurring taxa which may be highly competitive (Peay et al. 2007). Yet, the literature on the importance of competitive interactions in structuring endophyte communities, is sparse (Hassani et al. 2018), though this area is far more developed in the field of phytopathology (Veloso and Díaz 2012).

A particularly useful method to determine whether competitive interactions are leading to observable patterns of community composition is through co-occurrence analysis. One such analysis involves the generation of checkerboard scores, utilized by Stone and Roberts (1990) to describe a lack of co-occurrence of competing bird taxa in suitable habitat on the island nation of Vanuatu. A checkerboard is defined as two taxa that exist exclusively on only one of two sites or sampling units, such that a 1,0 and 0,1 is observed in an incidence matrix. However, more recently, checkerboard units have been 
applied to diverse systems including trees (Bar-Massada and Belmaker 2017), soil fungi (Kennedy et al. 2014) and marsupials (Ellis et al. 2009). When coupled with the generation of a null model (Wright et al. 1998; Gotelli and Entsminger 2003), the discernment of whether observed checkerboards are more frequent than would be expected by random chance becomes possible, making the method useful for generating inferences about broad community patterns. However, the application of checkerboard units has been slow to gain attention in endophyte community analyses (but see Sullivan and Faeth 2004 and Pan and May (2009). Clarifying the relative contribution of competitive interactions in shaping species' distributions has been a challenge for ecologists studying macroorganisms, and although determinations in cryptic and hyperdiverse microbes is even more challenging, developing an understanding in them is no less important. This is particularly evident when considering the ubiquitous nature of plant-associated microfungi and their potential effects on the distribution of plant communities in all terrestrial ecosystems (Arnold et al. 2000; Busby et al. 2015b).

In order to address the lack of awareness surrounding the relative importance of temporal turnover in structuring endophyte communities, we conducted an observational study leveraging the temperate fern, Polystichum munitum. Owing to its broad distribution across a large geographic range spanning coastal habitats, coniferous understories and montane habitats (Soltis and Soltis 1987; Limm and Dawson 2010), it is a particularly useful system to attempt to detect habitat-specific differences in endophyte community composition. Furthermore, although P. munitum is a long-lived host, new growth occurs each spring providing arriving colonists with potential habitat within host tissues. We specifically examined four unique habitats where populations of $P$. munitum 
existed, and also sampled the closest plants to each $P$. munitum sampled. We applied our sampling regime over three total days to account for potential temporal shifts in community composition and leveraged culture-independent amplicon sequencing on the Illumina MiSeq platform to draw conclusions regarding the following questions: (i) Is significant spatial turnover observed across the four sampling sites? (ii) Are there patterns of host specificity observed in either P. munitum or other neighboring plants? (iii) If there is a degree of host specificity, does this result in discernable patterns of competitive exclusion or the aggregation of certain co-occurring taxa? and (iv) What ecological principles may apply to observed patterns of spatial turnover and host specificity? To address these questions, we first examined differences in alpha and beta diversity, and then examined co-occurrence patterns through a quantification of the number of checkerboard units, comparing these values to two null models which generate random distributions. Further, we determined whether significant positive or negative correlations existed between highly abundant taxa and lastly examined taxonomic assignments to operational taxonomic units (OTUs) recovered from our sampling efforts. This is one of the first culture-independent studies of endophyte spatial turnover that employed an extensive sampling effort over a relatively large geographic distance (Cordier et al. 2012; Zimmerman and Vitousek 2012; Bazzicalupo et al. 2013) and one of the first cultureindependent studies conducted in endophyte communities of ferns (Younginger and Ballhorn 2017). The results provided herein demonstrate that dispersal limitation is not a significant factor in structuring fern endophyte communities as abundant taxa are observed in all four sites examined across $150 \mathrm{~km}$; however, endophytes which are not found in ferns seem limited in their potential to exploit fern host tissue through filtering 
and highly segregating community composition prevails between ferns and non-fern hosts.

Methods

Study sites and sampling protocol-

Samples were collected over three days from all four study sites to account for potential temporal effects on community composition (Chapter 2). The sites sampled in this study encompass the broad range of habitat conditions tolerated by the P. munitum host: (Ecola State Park; Coast) a coastal site on a rocky cliff with no tree canopy, 20 meters from the ocean at $45.92335 \mathrm{~N}$ and $123.97705 \mathrm{~W}$ and 28 meters above sea level, (Stub Stewart State Park; Coast Range) a coniferous understory habitat in the eastern foothills of the Oregon Coast Range at $45.733759 \mathrm{~N}, 123.18631 \mathrm{~W}$ and 310 meters above sea level (the same site sampled in the temporal study; Chapter 2), (Goat Marsh; MSH control) a coniferous understory site on the south slope of Mount Saint Helens, minimally impacted by the 1980 eruption at $46.15400 \mathrm{~N}, 122.27507 \mathrm{~W}$ and 907 meters above sea level and (Mount Saint Helens; MSH impacted) a secondary succession site with no tree canopy near Norway Pass impacted, by the 1980 eruption at $46.30437 \mathrm{~N}$ and 122.08801 W at 1155 meters above sea level. Plants were sampled on August $15^{\text {th }}$ (ESP and SSP) and August $17^{\text {th }}$ (GM and MSH), 2016 by snipping with fine-tipped gardening shears, dipped in ethanol and wiped clean with a fresh Kimwipe (Kimberly-Clark, TX, USA) between each sample. Samples were placed in a clean, unused coin envelope for transport to the laboratory. All samples were kept in a cooler and returned to the lab for processing within 8 hours of sampling. 
From each site, four Polystichum munitum pinnae (i.e. leaflets) were sampled from 10 plants $(\mathrm{n}=40)$ from fronds in the current year's growth. Additionally, two leaves or leaflets were obtained from each of the two closest plants to each fern sampled ( $\mathrm{n}=$ 40). Details on the taxonomy of the neighboring plants (hereafter "neighbors") is included in Appendix A. Thus, over three days, 320 total samples were collected from the four study sites. Surface sterilization and sample processing-

Samples were dipped in a $0.1 \%$ Tween solution and held under running tap water for $30 \mathrm{sec}$ to dislodge epiphytic material and then immersed in sequential baths of $95 \%$ ethanol, $0.5 \%$ sodium hypochlorite, and $70 \%$ ethanol (v/v; $10 \mathrm{sec}, 2 \mathrm{~min}, 2 \mathrm{~min}$, respectively; Arnold et al. 2003) in a sterile hood and allowed to dry on a fresh Kimwipe. One hundred mg of each sample was excised with a sterile scalpel and placed in sterile $1.7 \mathrm{~mL}$ Eppendorf tubes and stored at $-80^{\circ} \mathrm{C}$ until DNA extractions. Aliquots were taken from the final sterilization baths and included in DNA extractions and library preparation (below) and included in the sequencing run as negative controls (SS_Cont). DNA extraction and library preparation-

All samples were lyophilized and total DNA was extracted with the Qiagen DNeasy plant mini kit (Qiagen, Hilden, Germany) following the manufacturer's instructions. A negative control was included in each round of DNA extractions and the elutions were then pooled and included in a single negative control for the sequencing run (Ex_Cont). Extraction success was verified in replicate PCR reactions utilizing the primers ITS1F and ITS4 (White et al. 1990). PCR reactions contained $12.5 \mu \mathrm{L}$ of 10X GoTaq PCR Master Mix (Promega, WI, USA), $1.25 \mu \mathrm{L}$ of $10 \mathrm{mg} / \mathrm{mL}$ BSA, $1.25 \mu \mathrm{L}$ of 
each primer at $10 \mu \mathrm{M}, 1 \mu \mathrm{L}$ of template DNA, and diluted up to $25 \mu \mathrm{L}$ with PCR water. Thermocycler conditions were as follows: $94^{\circ} \mathrm{C}$ initial denaturation for 3 min and 35 cycles of $94^{\circ} \mathrm{C}$ for $1 \mathrm{~min}, 50^{\circ} \mathrm{C}$ for $1 \mathrm{~min}, 72^{\circ} \mathrm{C}$ for $1 \mathrm{~min}$ with a final elongation at $72^{\circ}$ $\mathrm{C}$ for 10 minutes. Reactions were held at $4^{\circ} \mathrm{C}$ until removed from the thermocycler and verified with gel electrophoresis. The template DNA of any samples which failed to amplify in both replicates of initial PCR were cleaned with the Agencourt AMPure XP kit (Beckman Coulter, CA, USA) to remove potential PCR inhibitors and then verified with the same PCR reaction conditions. Only 33 samples were cleaned with the AMPure kit (all neighboring plants; not $P$. munitum) and of these cleaned samples, only 9 failed to amplify in initial PCR. All samples were then included in library preparation steps, regardless of initial PCR success.

For the library preparation steps, fungal DNA was amplified in duplicate with the fungal primers ITS1F and ITS2 that also contained Illumina MiSeq adaptor sequences and a 12 bp unique barcode on the reverse primer for each sample (Smith and Peay 2014). Reactions contained $5 \mu \mathrm{L}$ of $5 \mathrm{X}$ Phusion High Fidelity buffer, $0.5 \mu \mathrm{L}$ of $10 \mathrm{mM}$ dNTPs, $0.5 \mu \mathrm{L}$ of each primer at $10 \mu \mathrm{M}, 0.63 \mathrm{U}$ of Phusion High Fidelity polymerase (New England BioLabs, MA, USA), $1 \mu \mathrm{L}$ of template DNA (5-30 ng/ $\mu \mathrm{L})$, and diluted up to $25 \mu \mathrm{L}$ with PCR water. Thermocycler conditions were as follows: $94^{\circ} \mathrm{C}$ initial denaturation for $60 \mathrm{sec}$ and $30 \mathrm{cycles}$ of $94^{\circ} \mathrm{C}$ for $30 \mathrm{sec}, 52^{\circ} \mathrm{C}$ for $30 \mathrm{sec}$, and $68^{\circ} \mathrm{C}$ for $30 \mathrm{sec}$ with a final elongation at $68^{\circ} \mathrm{C}$ for 7 minutes. Reactions were held at $4^{\circ} \mathrm{C}$ until removed from the thermocycler and verified with gel electrophoresis. Each PCR included a negative control with the same reverse primer barcode (PCR water) and all of these controls were pooled and included in the sequencing run (PCR_Cont). Duplicate products 
from library preparation PCR were pooled and cleaned with the AMPure kit and final concentrations of cleaned PCR products were verified with the Qubit Fluorometer (Life Technologies, CA, USA) according to the manufacturer's instructions. The 320 samples were split into two sequencing runs to ensure enough sequencing depth. Each run consisted of half of the samples, the SS_Cont, Ex_Cont, PCR_Cont and a mock community as a positive control, comprised of 30 taxa of known taxonomy and concentration (MC). Ten ng of each cleaned sample was added to the final library, diluted to $10 \mathrm{nM}$ and sequenced on the Illumina MiSeq platform $2 \times 250 \mathrm{bp}$ at the Oregon State Center for Genome Research and Biocomputing with a 30\% PhiX spike added to each sequencing run.

Sequence processing and bioinformatics-

An examination of read quality from both the forward and reverse reads revealed that the reverse reads were of much lower quality and may result in the loss of many viable forward sequences if merging were attempted (Nguyen et al. 2014). Therefore, we chose to analyze this dataset with the forward reads only. Demultiplexed sequence headers were relabeled with sample IDs through a custom shell script and the fastx_relabel command of USEARCH v10.0.240 (Edgar and Flyvbjerg 2015) and primer sequences were removed with Cutadapt v1.10 (Martin 2011). A total of 9.0 M sequences were recovered from both sequencing runs and included in this study. Sequences were denoised into zero-radius operational taxonomic units (zOTUs; also known as ESVs but hereafter called OTUs; Callahan et al. 2017) by first trimming the forward reads to a fixed length (180 bp), removing any sequences with a sum of error probabilities (Qscores from Illumina runs) $\geq 1.0$ (Edgar and Flyvbjerg 2015), identifying unique 
sequences, and then grouping together these unique sequences into OTUs through the UNOISE3 command (Edgar 2016b) by correcting sequence errors and removing chimeras. The original sequences (with the primers removed) were then mapped back to these OTUs with $88.1 \%$ mapping correctly. Taxonomic assignments were called with the SINTAX algorithm (Edgar 2016a) using the most recent version of the UNITE ITS database (v01.12.2017; Kõljalg et al. 2013) and added to the OTU matrix. Control samples were removed from the final OTU matrix by subtracting the total number of reads of all controls from the actual samples (Nguyen et al. 2014; Younginger and Ballhorn 2017). The resultant OTU matrix and associated metadata were imported into R v3.3.2 ( $\mathrm{R}$ core team 2016) and analyzed with the packages phyloseq v1.19.1 (McMurdie and Holmes 2014) and vegan v2.4.2 (Oksanen et al., 2011) for statistical analyses and figures were generated with ggplot2 v2.2.1 (Wickham 2009).

Statistics-

Sequences for each plant were pooled computationally (Song et al. 2015) and rarefied to 1090 sequences per sample by randomly sampling with replacement. This resulted in the loss of 2 samples (both from Coast neighbors). Alpha diversity metrics were calculated with Hill numbers at the scales of $q=0$, 1, and 2 (Hill 1973; Chiu and Chao 2016) and boxplots were generated from these values in ggplot2. Significant differences in each alpha diversity measure (OTU richness, the exponential of Shannon entropy and the inverse of Simpson index) were examined with the Kruskal-Wallis rank sum test (Kruskal and Wallis 1952) and significant differences between groups were detected with Dunn's test of multiple comparisons with a Benjamini-Hochberg correction (Dunn 1964; Benjamini and Hochberg 1995). Community composition was visualized 
through non-metric multidimensional scaling (NMDS) with a square root transformation and Wisconsin double standardization following the generation of a Bray-Curtis dissimilarity matrix. Significant differences between sampling months were examined with a permutational multivariate analysis of variance (PERMANOVA; Anderson 2001, Anderson and Walsh 2017) following 999 permutations with Bray-Curtis dissimilarity. Additionally, significant differences between the dispersion of group centroids (corresponding to each group: sampling site and ferns versus neighbors) was determined through a multivariate version of Levene's test for variance homogeneity (Anderson 2006).

In order to examine whether the presence of certain taxa significantly affected the occurrence of other taxa, we examined the number of checkerboard units through the oecosimu function in vegan (Oksanen et al. 2011). The OTU table of sequencing read numbers was first converted to a binary incidence matrix and within each sampling site (ferns plus neighbors) the number of checkerboard units were determined (i.e. a $2 \times 2$ submatrix, where two taxa occur in a 1,0 and 0,1 pattern in different plants; Stone and Roberts 1990). This yields a C-score which represents the degree of randomness or structure to community composition (lower values equal more randomness). The results were then compared with two null models: one which preserved the total number of OTU incidences within each plant, but used the total frequency of each OTU as a probability for refilling values ( $r 1$; Wright et al. 1998) and a second which preserved the frequencies of both the rows and columns, but shuffled OTU frequencies within shuffleboards that existed in the matrix (swap; Gotelli and Entsminger 2003). Both null models were run for 999 iterations and were then compared to checkerboard scores to generate test statistics. 
Additionally, Pearson correlation coefficients were calculated on the top 10 OTUs within each study site — sorted by abundance — and also on the top 10 OTUs from the total dataset with the cor function in R. All correlations were tested for significance with a Pearson's product moment correlation test (cor.test in R) without a correction as no probability values were deduced from multiple tests; only pairwise correlations were examined. Finally, sequencing read numbers were converted to relative abundance and any taxa that were represented in less than $5 \times 10^{-4}$ of the total dataset were removed for easier visualization in a taxonomy plot.

Results

Alpha diversity-

A similar trend is observed across all three alpha diversity metrics (Hill numbers 0,1 and 2), which place increasing emphasis on common taxa as the Hill number increases in value (Figure 3.1). The richness ( $\mathrm{q}=0)$ in P. munitum microbial communities is significantly greater at the Coast than at the Coast Range or the MSH impacted sites, but not greater than at MSH control site-a site close to MSH impacted. When comparing neighboring plants at each study site to $P$. munitum, a significant difference is seen in microbial communities in all study sites except for MSH impacted. At the Coast, MSH control and MSH impacted sites, there is a greater richness of endophyte OTUs in $P$. munitum than in neighboring plants, though not significant at MSH impacted. The greatest difference in richness at a single study site is found between $P$. munitum and neighboring plants at the Coast Range. When $\mathrm{q}=1$ (exponential of Shannon entropy), 


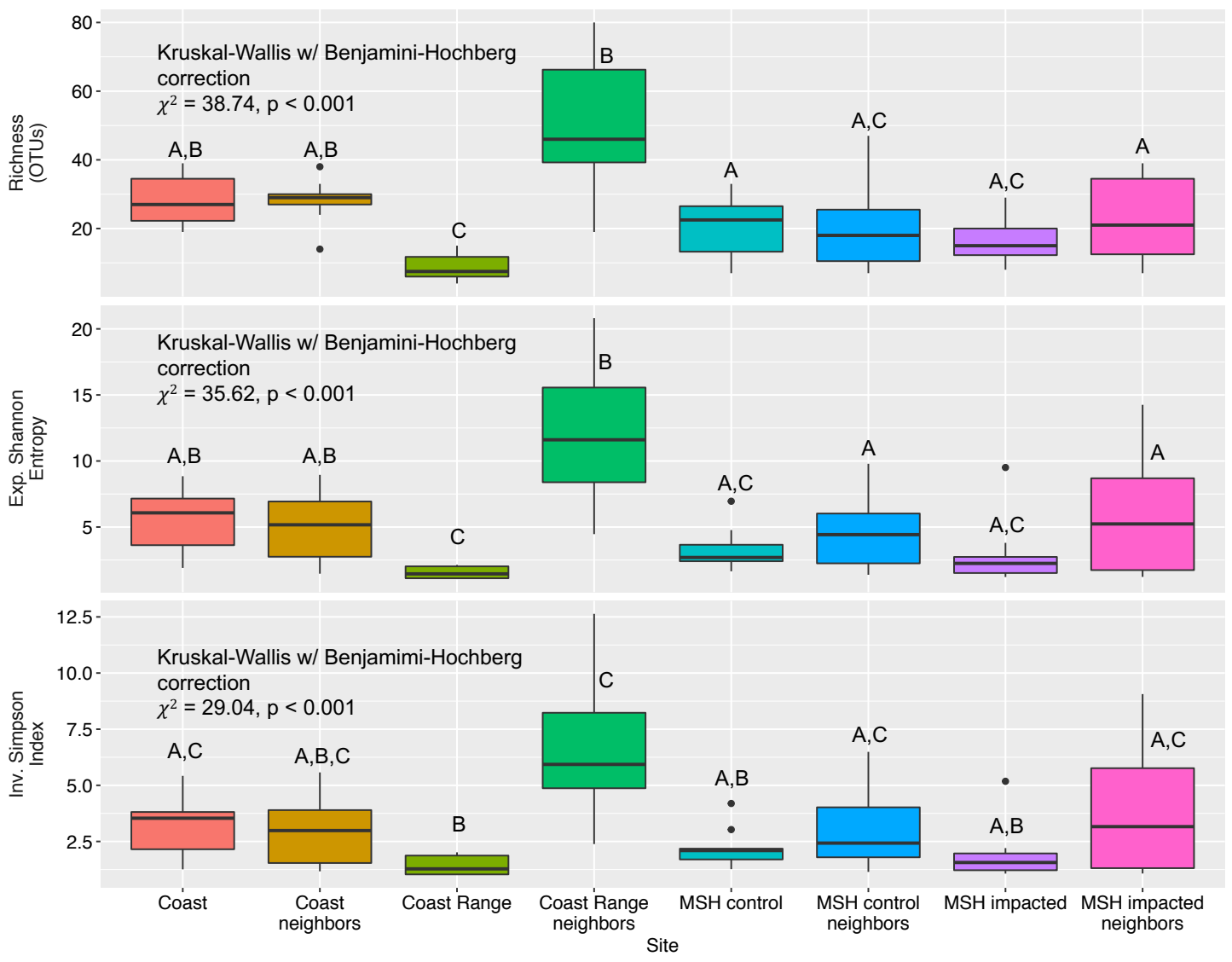

Figure 3.1 Hill measures of alpha diversity at the scales of $q=0,1$ and 2 within each sampling site, separated by endophyte communities colonizing the Polystichum munitum host and endophytes colonizing the plants growing in closest proximity to the $P$. munitum host (neighbors). As the value of $\mathrm{q}$ increases, the alpha diversity measure becomes less sensitive to rare taxa. Significant differences in overall Hill measures were examined with the Kruskal-Wallis rank sum test and significant differences between groups were examined with Dunn's test of multiple comparisons, including a BenjaminiHochberg correction. Boxplots represent the median value (middle line) of each alpha diversity measurement within each group, boxes represent upper and lower quartiles and lines represent upper and lower deciles $(\mathrm{n}=10$ plants per facet). The MSH initialism represents Mount Saint Helens.

there is significantly more diversity seen in P. munitum hosts at the Coast and MSH

control sites than at the Coast Range. A comparison of $P$. munitum and neighbors within each site demonstrates greater diversity in P. munitum at the Coast and MSH control (not significantly different), but lower diversity in P. munitum hosts at the Coast Range and MSH impacted, though not statistically different at MSH impacted. Similar results are 
found between study sites and within study sites when $\mathrm{q}=2$ (inverse of Simpson index) as seen when $\mathrm{q}=1$.

Beta diversity/ordination-

When compressing community composition into two axes through NMDS ordination, we observe that $P$. munitum hosts are more similar in community composition across all study sites than they are to any neighbors within the same study site (Figure 3.2). Further, P. munitum plants within each study site have more similar microbial communities to each other than neighboring plants have to each other, with the exception of the Coast neighbors which also appear relatively close in community composition, as demonstrated by the compressed $95 \%$ confidence ellipses. Finally, a degree of nestedness is found in MSH control microbial communities of P. munitum when compared to those of neighbors, though no points are found within either group's 95\% confidence ellipses. Overall, there is overlap in all 95\% confidence intervals, yet PERMANOVA results demonstrate a significant difference between sampling units (ferns versus neighbors within each site). Furthermore, there is a significant difference in the degree of group dispersions found with the permutation-based Levene's test.

Co-occurrence patterns-

A significant difference was found between the observed number of checkboards and those detected in null models for all sites examined (including the full dataset), except for the MSH impacted site (Table 3.1). C-score values did not differ substantially between sites, nor show discernable patterns with observed p-values when compared to null models; however, the Coast Range site had a far greater number of checkerboards (between 2.10- and 2.43-fold) relative to other sites and also had the highest standardized 
effect sizes (SES) when compared to both null models of all groups (including the full

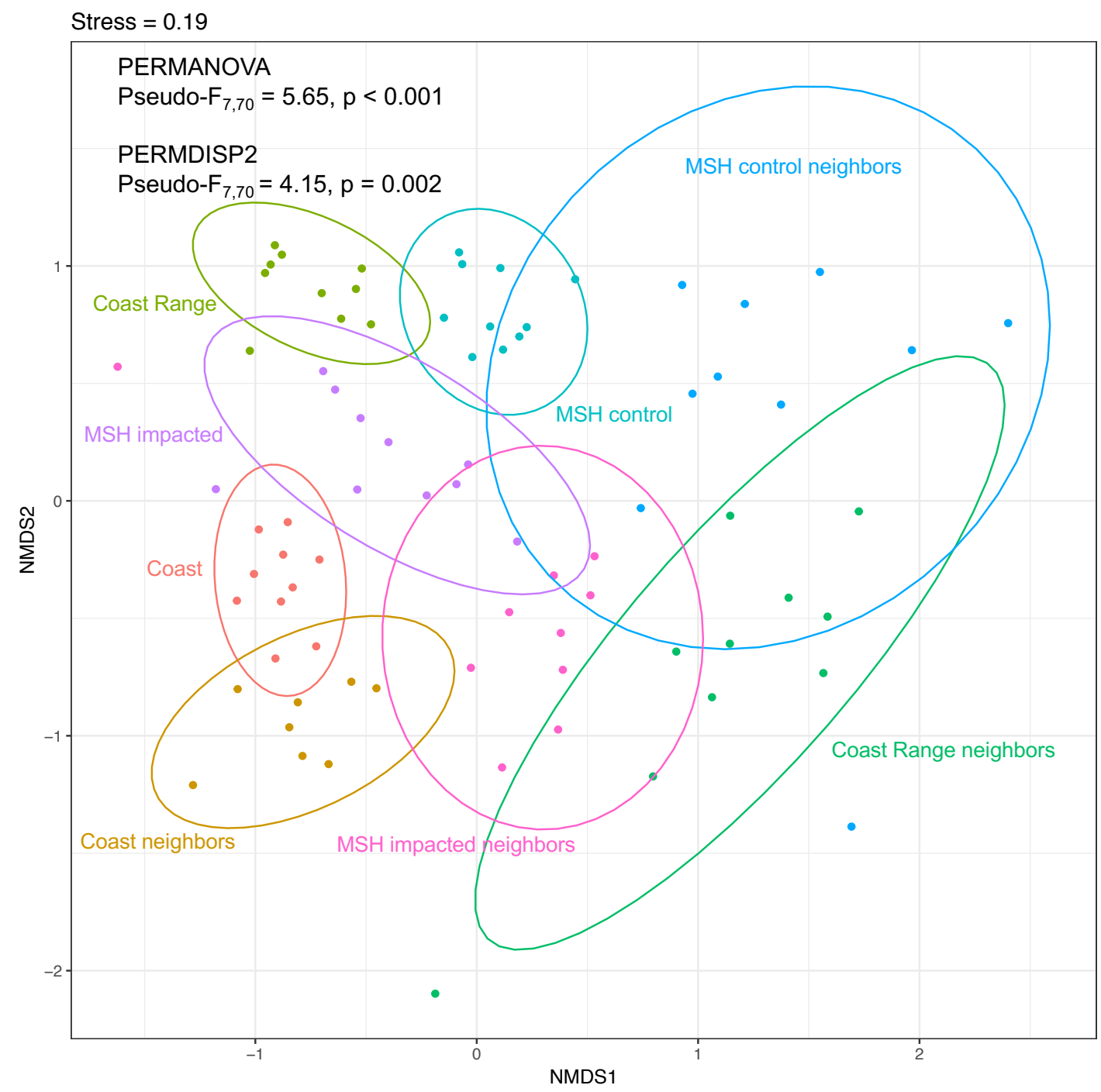

Figure 3.2 Non-metric multidimensional scaling (NMDS) of endophyte community composition across the full dataset. Values were square root transformed and Wisconsin double standardized following the generation of a Bray-Curtis dissimilarity matrix from the OTU table. Points represent plants at each study site, colored by the site and whether they came from the Polystichum munitum host or a neighboring plant to P. munitum. Ellipses represent 95\% confidence intervals and stress is equal to 0.19. Differences in community composition throughout the sampling period were examined with a repeatedmeasures permutational multivariate analysis of variance (PERMANOVA) following 999 permutations of Bray-Curtis dissimilarity. Significant differences in group dispersion about each centroid were tested with a multivariate version of Levene's test for variance homogeneity (PERMDISP $2 ; n=10$ plants per group).

dataset). Also, the MSH impacted site had the lowest number of checkerboards and SES-

values among all sites studied. 
Table 3.1 Checkerboard analysis of OTU incidences within each site (not separated by host type) and examined against two null models for significant differences. The number of checkerboards corresponds to two OTUs and two plants in which a 1,0 and 0,1 are observed in the incidence matrix. The null model $r l$ preserved incidence frequencies within plants and used OTU frequencies as probabilities for refilling the matrix while the null model swap preserved frequencies of both OTUs and plants while shuffling $2 \times 2$ checkboards within the matrix. Each null model was run for 999 iterations and compared to observed checkerboard scores to generate probabilities. C-scores represent the average number of checkerboards observed for each unique pair of OTUs in each site, with lower values indicating more randomness and higher values indicating competitive exclusion. Standardized effect sizes (SES) represent co-occurrence patterns that are either highly segregated (positive values) or aggregated (negative values) with values close to zero denoting random co-occurrence patterns. Pvalues represent the probabilities of observed checkboard units differing from the null models by random chance and values $\leq 0.05$ were deemed significant (bold).

\begin{tabular}{cccccc} 
Plots & Null model method & Number of checkerboards & C-score & SES & P-value \\
\hline Coast & $r 1$ & 52921 & 2.80 & -3.81 & $\mathbf{0 . 0 0 1}$ \\
& swap & & & 2.09 & $\mathbf{0 . 0 4 9}$ \\
Coast range & $r 1$ & 119710 & 1.86 & 6.37 & $\mathbf{0 . 0 0 1}$ \\
& swap & & & 4.36 & $\mathbf{0 . 0 0 7}$ \\
MSH control & $r 1$ & 57048 & 2.12 & 2.31 & $\mathbf{0 . 0 2 5}$ \\
& swap & & & 3.81 & $\mathbf{0 . 0 0 3}$ \\
MSH impacted & $r 1$ & 49167 & 2.00 & 0.69 & 0.485 \\
& swap & & & 1.95 & $\mathbf{0 . 0 3 9}$ \\
Full dataset & $r 1$ & 1521527 & 4.81 & 4.89 & $\mathbf{0 . 0 0 1}$ \\
& swap & & & 2.50 & $\mathbf{0 . 0 0 1}$
\end{tabular}

The pairwise correlation analysis revealed a total of 16 negative and 21 positive correlations that were significant between plants within all study sites (excluding the full dataset; Table 3.2). In the full dataset, the presence of more abundant OTUs (i.e. lower OTU numbers) was positively associated with significant correlations between the presence of other abundant taxa. Although OTUs included in correlation analysis (the top 10 OTUs within each site and also within the full dataset) were more abundant relative to other rare OTUs not included in this analysis, lower OTU numbers tended be negatively associated with other lower OTU numbers in full dataset. When comparing significant correlations within each study site, the Coast Range site demonstrated the most significant positive and negative correlations. This was particularly evident with OTU2 
(Claussenomyces), which was the most abundant OTU at the study site and most often negatively correlated with the presence of other taxa (except for OTU7, Cronartium comptoniae). Less abundant taxa were more often positively associated with each other to a significant degree at the Coast Range site. The MSH control site also had a higher number of significant correlations relative to other sites, and also revealed negative correlations between OTU2 and other taxa (except for OTU4, Claussenomyces_sp.2). Very few positive or negative interactions were significant at the MSH impacted site, although the presence of the top three OTUs within the site were positively correlated with each other. The correlation analysis aligns with the results seen in the checkerboard analysis in that sites with more checkerboards and lower probabilities between null models (e.g. Coast Range and MSH control) also had more significant correlations between abundant taxa within each site. Notably, three of the top 10 taxa at the MSH control site were assigned to the Claussenomyces genus, yet further inspection of their representative sequences revealed that they were indeed different genotypes.

\section{Taxonomy-}

Trends seen in ordination and the correlation analysis are additionally reinforced when plotting the taxonomic assignments of the OTUs. Specifically, the taxonomy of endophyte communities in fern hosts appears more similar across all four study sites than they do in neighboring plants that are close in proximity (Figure 3.3). Additionally, the most geographically distant sites to one another, the Coast and MSH impacted sites respectively, seem to share more genera in common and the Coast Range and MSH control sites also seem to share more genera in common among fern hosts. This trend is similarly seen between study sites of neighboring plants, though more loosely so. 
Table 3.2 Pearson correlation coefficients of the top 10 OTUs within each site or the full dataset calculated from incidence matrices. Taxonomic assignments made to each OTU are included in the first column along with respective OTU numbers which are also denoted in the column headers. Bold correlation coefficients are significant, detected through Pearson's product moment correlation tests on each pairwise comparison $(\mathrm{P}<0.05)$.

\begin{tabular}{|c|c|c|c|c|c|c|c|c|c|c|}
\hline Full & OTU 1 & OTU 2 & OTU 3 & OTU 4 & OTU 7 & OTU 9 & OTU 13 & OTU 14 & OTU 19 & OTU 38 \\
\hline 1. Eleutheromyces pseudosubulatus & 1 & & & & & & & & & \\
\hline 2. Claussenomyces sp. & 0.47 & 1 & & & & & & & & \\
\hline 3. Piskurozyma cylindrica & 0.50 & 0.50 & 1 & & & & & & & \\
\hline 4. Claussenomyces sp. 2 & 0.28 & 0.67 & 0.47 & 1 & & & & & & \\
\hline 7. Cronartium comptoniae & -0.02 & 0.24 & 0.17 & 0.07 & 1 & & & & & \\
\hline 9. Neoerysiphe nevoi & -0.02 & -0.12 & -0.12 & -0.22 & 0.06 & 1 & & & & \\
\hline 13. Nodulosphaeria aconiti & 0.23 & 0.00 & 0.12 & -0.13 & 0.03 & 0.34 & 1 & & & \\
\hline 14. Preussia sp. & 0.02 & -0.02 & -0.06 & 0.23 & -0.23 & -0.14 & 0.01 & 1 & & \\
\hline 19. Filobasidium oeirense & -0.04 & -0.14 & 0.01 & -0.06 & -0.15 & -0.15 & -0.28 & -0.37 & 1 & \\
\hline 38. Stemphylium vesicarium & -0.04 & -0.11 & 0.02 & -0.11 & -0.19 & 0.01 & -0.21 & -0.33 & 0.80 & 1 \\
\hline Coast & OTU 1 & OTU 2 & OTU 3 & OTU 19 & OTU 28 & OTU 34 & OTU 36 & OTU 40 & OTU 82 & OTU 109 \\
\hline 1. Eleutheromyces pseudosubulatus & 1 & & & & & & & & & \\
\hline 2. Claussenomyces sp. & 0.56 & 1 & & & & & & & & \\
\hline 3. Piskurozyma cylindrica & 0.32 & 0.16 & 1 & & & & & & & \\
\hline 19. Filobasidium oeirense & -0.20 & -0.25 & 0.00 & 1 & & & & & & \\
\hline 28. Sporobolomyces roseus & -0.39 & -0.01 & -0.35 & 0.39 & 1 & & & & & \\
\hline 34. Golovinomyces montagnei & -0.11 & -0.19 & -0.17 & 0.11 & -0.39 & 1 & & & & \\
\hline 36. Stagonospora pseudovitensis & 0.32 & -0.16 & 0.00 & 0.00 & -0.18 & -0.34 & 1 & & & \\
\hline 40. Neostagonospora elegiae & 0.45 & 0.11 & -0.24 & 0.15 & -0.12 & 0.24 & 0.47 & 1 & & \\
\hline 82. Sclerotinia sclerotiorum & 0.12 & 0.12 & -0.38 & -0.48 & 0.03 & -0.13 & 0.09 & 0.00 & 1 & \\
\hline 109. Phomatodes aubrietiae & -0.36 & 0.30 & -0.08 & 0.05 & 0.24 & -0.19 & -0.40 & -0.34 & -0.15 & 1 \\
\hline Coast Range & OTU 2 & OTU 7 & OTU 11 & OTU 12 & OTU 60 & OTU 73 & OTU 75 & OTU 87 & OTU 90 & OTU 105 \\
\hline 2. Claussenomyces sp. & 1 & & & & & & & & & \\
\hline 7. Cronartium comptoniae & 0.58 & 1 & & & & & & & & \\
\hline 11. Leohumicola minima & -0.58 & -0.33 & 1 & & & & & & & \\
\hline 12. Symbiotaphrina buchneri & -0.42 & -0.24 & 0.4 & 1 & & & & & & \\
\hline 60. Orbilia alba & -0.52 & -0.42 & 0.79 & 0.57 & 1 & & & & & \\
\hline 73. Plectania melastoma & -0.65 & -0.38 & 0.63 & 0.34 & 0.66 & 1 & & & & \\
\hline 75. Symbiotaphrina kochii & -0.65 & -0.38 & 0.38 & 0.34 & 0.43 & 0.29 & 1 & & & \\
\hline 87. Everhartia hymenuloides & -0.82 & -0.47 & 0.71 & 0.51 & 0.68 & 0.8 & 0.58 & 1 & & \\
\hline 90. Didymella boeremae & -0.42 & -0.24 & 0.4 & 0.22 & 0.28 & 0.03 & 0.34 & 0.23 & 1 & \\
\hline 105. Symbiotaphrina buchneri sp. 2 & -0.42 & -0.24 & 0.4 & 0.61 & 0.28 & 0.03 & 0.03 & 0.23 & 0.22 & 1 \\
\hline
\end{tabular}


Table 3.2 Cont.

\begin{tabular}{|c|c|c|c|c|c|c|c|c|c|c|}
\hline MSH control & OTU 1 & OTU 2 & OTU 4 & OTU 5 & OTU 6 & OTU 10 & OTU 11 & OTU 14 & OTU 17 & OTU 104 \\
\hline 1. Eleutheromyces pseudosubulatus & 1 & & & & & & & & & \\
\hline 2. Claussenomyces sp. & 0.42 & 1 & & & & & & & & \\
\hline 4. Claussenomyces sp.2 & 0.42 & 1.00 & 1 & & & & & & & \\
\hline 5. Claussenomyces olivaceus & -0.14 & -0.33 & -0.33 & 1 & & & & & & \\
\hline 6. Lachnellula flavovirens & -0.21 & -0.50 & -0.50 & -0.17 & 1 & & & & & \\
\hline 10. Articulospora sp. & -0.21 & -0.50 & -0.50 & 0.25 & 0.06 & 1 & & & & \\
\hline 11. Leohumicola minima & -0.24 & -0.12 & -0.12 & 0.19 & 0.29 & 0.00 & 1 & & & \\
\hline 14. Preussia sp. & 0.18 & 0.42 & 0.42 & -0.33 & 0.21 & -0.49 & -0.08 & 1 & & \\
\hline 17. Alysidiella parasitica & 0.23 & 0.61 & 0.61 & -0.27 & -0.15 & -0.41 & 0.24 & 0.34 & 1 & \\
\hline 104. Articulospora sp.2 & -0.21 & -0.50 & -0.50 & 0.25 & 0.06 & 0.06 & 0.00 & -0.49 & -0.41 & 1 \\
\hline MSH impacted & OTU 1 & OTU 2 & OTU 3 & OTU 9 & OTU 13 & OTU 14 & OTU 18 & OTU 20 & OTU 151 & OTU 286 \\
\hline 1. Eleutheromyces pseudosubulatus & 1 & & & & & & & & & \\
\hline 2. Claussenomyces sp. & 0.73 & 1 & & & & & & & & \\
\hline 3. Piskurozyma cylindrica & 0.60 & 0.70 & 1 & & & & & & & \\
\hline 9. Neoerysiphe nevoi & -0.25 & -0.22 & -0.37 & 1 & & & & & & \\
\hline 13. Nodulosphaeria aconiti & -0.31 & -0.23 & -0.25 & -0.35 & 1 & & & & & \\
\hline 14. Preussia sp. & -0.10 & -0.31 & -0.03 & -0.25 & 0.17 & 1 & & & & \\
\hline 18. Podosphaera astericola & -0.24 & -0.33 & -0.30 & 0.15 & 0.08 & -0.24 & 1 & & & \\
\hline 20. Stagonospora perfecta & -0.24 & -0.33 & -0.30 & 0.15 & 0.08 & 0.45 & -0.11 & 1 & & \\
\hline 151. Leohumicola minima & -0.24 & -0.33 & -0.30 & 0.15 & 0.08 & 0.10 & 0.44 & -0.11 & 1 & \\
\hline 286. Symbiotaphrina buchneri & -0.17 & -0.23 & -0.21 & -0.15 & 0.05 & 0.31 & -0.08 & -0.08 & -0.08 & 1 \\
\hline
\end{tabular}




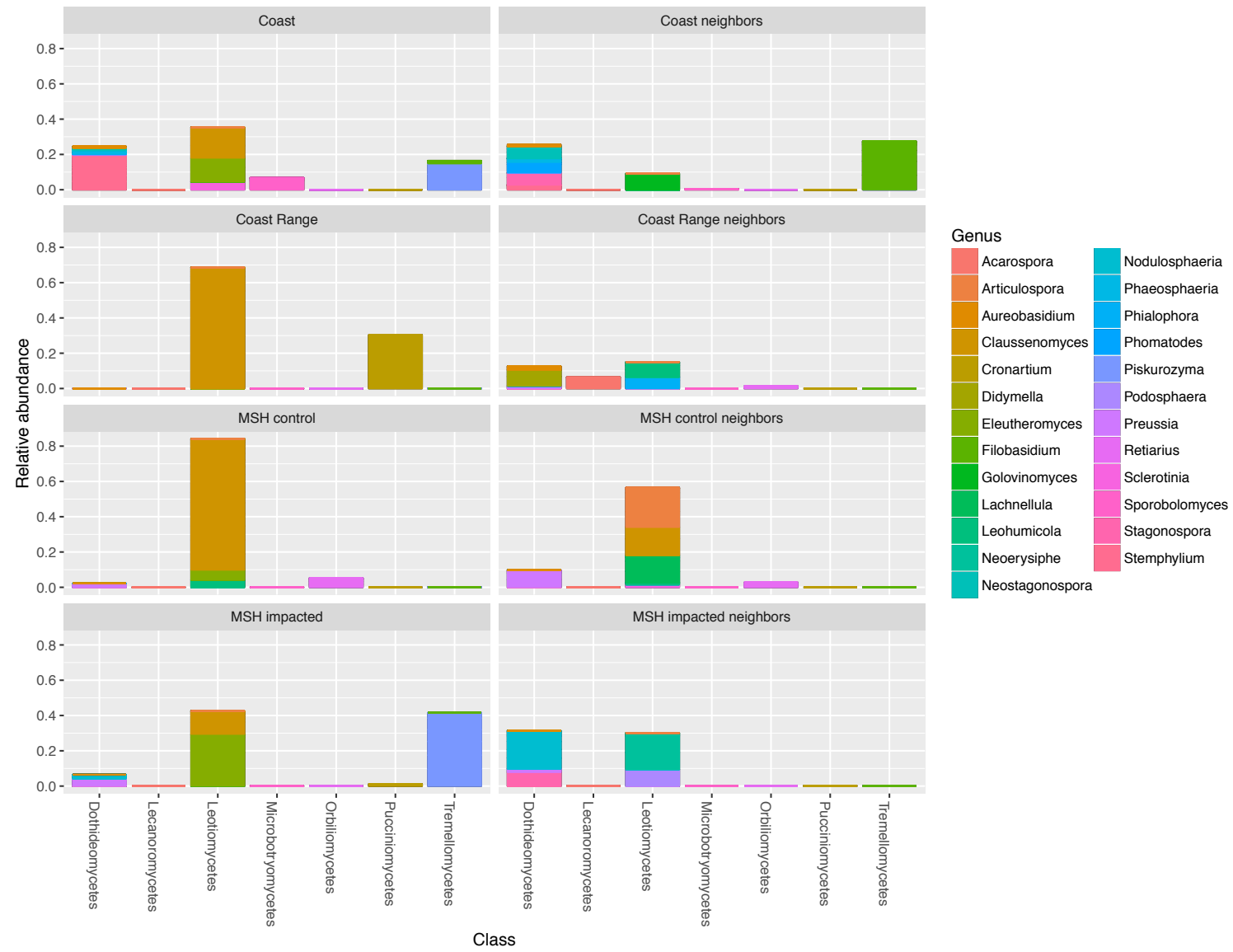

Figure 3.3 Taxonomy plot of endophyte community composition within site, separated by the Polystichum munitum host and neighboring plants. Colored bars represent the relative abundance of genera found within each fungal class (x-axis). Any taxa that represent less than $5 \times 10^{-4}$ of the total dataset were removed for easier visualization $(n=10$ plants per facet).

\section{Discussion}

Fungal endophyte communities that colonize Polystichum munitum are remarkably unique relative to neighboring host plants growing in close proximity. There are more taxa shared in $P$. munitum hosts across sites that are up to $150 \mathrm{~km}$ away than in plants that are competing for shared resources - and often physically contacting each otherwithin the same community. Although a strong effect of distance-decay is presumed to be a driver of high beta and gamma diversity in microbial communities (Meiser et al. 2014), we find little evidence of this occurring in our dataset, especially among $P$. munitum 
hosts. Instead, it appears that habitat filtering is facilitated by the fern host, enabling colonists optimized for a symbiotrophic lifestyle within $P$. munitum to persist. Furthermore, if host tissue is considered a habitat which creates very specific environmental conditions for its symbionts (which is likely), these patterns are indicative of species sorting (Van der Gucht et al. 2007) occurring within small areas of potential habitat.

Significantly lower endophyte richness and diversity is observed in P. munitum hosts than in neighboring plants at the Coast Range site, likely due to the presence of OTU2. This same OTU is found in most of the fern hosts in all four sites, and a similar reduction in diversity in ferns endophytes (but not richness) is also observed in the MSH control and MSH impacted sites, though not significantly different. Surprisingly, the greatest richness and diversity measured across all sites was seen in the Coast Range neighbors, growing in the immediate vicinity of the $P$. munitum hosts with the lowest endophyte richness and diversity. This strong signal of contrasting richness and diversity found in different host species is further analyzed in the co-occurrence analysis (below), but is evidence of highly segregating endophyte communities: certain taxa exclusively colonize $P$. munitum and other taxa exclusively colonize neighboring plants, with little overlap of taxa colonizing both ferns and neighboring hosts. At the two Mount Saint Helens sites (MSH control and MSH impacted), significant differences are not observed in all three alpha diversity measures between ferns and neighbors, but the diversity in ferns $(q=1$ and 2$)$ is lower, likely due to the presence of fewer common taxa or an uneven community between fern hosts. Also notable is the lack of difference in all three 
alpha diversity measures at the Coast site between ferns and neighbors, as this site is the closest geographically to the Coast Range where the greatest differences were observed. We acknowledge that samples obtained from fern neighbors were more taxonomically diverse (from the perspective of the host plant) than from ferns and could confound inferences made from alpha diversity estimates. Additionally, sequencing reads were pooled from two different neighbors per fern (and these were often different plant species) potentially magnifying this issue; however, diversity measures were not significantly different between the ferns and neighbors at three of the four sites. In the Coast Range site, which did exhibit a significant difference in alpha diversity between ferns and neighbors, we also find stronger patterns of competitive exclusion when examined in the co-occurrence analysis (below). Therefore, we are confident that our sampling and pooling protocol still accurately reflects microbial community composition at each site, especially from the perspective of the $P$. munitum hosts which were the focus of this study.

The NMDS ordination demonstrates that community composition is relatively similar between $P$. munitum hosts within a study site (i.e. lower dispersion, though less so at MSH impacted) and also between P. munitum hosts across study sites (i.e. confidence interval overlap). In contrast, community composition between neighbor plants within a site is less similar, as seen in the larger dispersions of these groups relative to P. munitum communities. Although a significant difference in community composition was detected in PERMANOVA, we cannot rule out the fact that differences in dispersion-also significant in the Levene's test-are leading to this result as overlap is observed in every 
95\% confidence ellipsis with at least one other group. Also, all P. munitum and neighbor groups within each study site have overlap in their 95\% confidence ellipses with the exception of the Coast Range, which is indicative of some shared endophyte community members. Still, the pattern of higher community similarity between ferns both within and across study sites is noteworthy, particularly when considered in the context of the close proximity of the neighbor plants to the ferns within each site. It appears likely that $P$. munitum is a suitable host for a select number of endophyte taxa that represent a small proportion of the regional species pool (Kneitel and Miller 2003). Potential mechanisms for this pattern - from the perspective of the host—could occur through the production of high levels of tannins or low rates of herbivory (as herbivory could serve as a vector of fungal inocula). We did not directly measure tannins or herbivory in the present work, but P. munitum is rarely antagonized in nature by herbivores or pathogens (pers. observation) making these scenarios possible, but speculative. Alternatively, the microbial colonists detected in ferns in this work could be altering the suitability of host tissue for colonization by competitors through niche preemption or niche modification (i.e. priority effects; Fukami 2015) and this is one of the potential mechanisms for the sharp temporal turnover seen in Chapter 2. Furthermore, it would be interesting to incorporate a phylogenetic distance in the construction of an ordination plot to account for endophyte relatedness, since several abundant OTUs in fern hosts were assigned to the genus Claussenomyces within the Pseudeurotiaceae (all were unique genotypes). Unfortunately, the fungal ITS region is a notably poor estimator of evolutionary history, despite its usefulness as a barcode (Bruns 2001; Nilsson et al. 2008; Vellinga et al. 2015). 
The checkerboard analysis highlighted several co-occurrence patterns in the present work that are notable. First, the greatest number of checkerboards within all study sites was observed in the Coast Range. This is intuitively clear since there was both the highest and lowest richness observed across all sites in the neighbors and ferns, respectively. Despite this high number of checkerboards, the Coast site also had the lowest average number of checkerboards per unique species pair (C-score). This is most likely driven by the fact that there were few checkerboards observed when comparing taxa in fern hosts—since they were more homogeneous—yet more checkerboards observed in non-fern hosts - due to greater richness. Stated differently, if two taxa were observed in two different ferns, they are less likely to exist as a checkerboard due to the high host-specificity. This segregation of microbial taxa in the Coast Range study site is further emphasized by the relatively high standardized effect sizes (SES), as values far from zero indicate less randomness.

The lowest number of checkerboards, observed in the MSH impacted site, is indicative of more random or evenly dispersed microbial communities. In fact, SES values were close to zero when compared to both null models, indicating high randomness and a non-significant difference from the $r 1$ null model. This result may be explained through the stress gradient hypothesis, which predicts that competitive interactions are dampened in stressful environments (Bertness and Callaway 1994). Almost 38 years have elapsed since secondary succession began at the MSH impacted site following the 1980 eruption, but when considered in the context of suitable habitats for microbial communities, may still be a stressful habitat in which to exist because of 
high ultraviolet radiation and temperature fluctuations; common in montane habitats without canopy cover. Lastly, although significant differences were found in the number of checkerboards when compared to null models from the Coast site, the SES values indicated strong aggregation (from the $r 1$ null model) and moderate segregation (from the swap null model) with a marginally significant difference. Although the Coast site is close to sea level and $150 \mathrm{~km}$ from the MSH impacted site, it more closely resembles many physical attributes from the MSH impacted site (e.g. no canopy cover, and a higher frequency of Poa spp. neighbors). These two sites are also more similar in community composition when viewed in ordination, a fact that deserves more attention in future work.

The checkerboard co-occurrence analysis was able to characterize broad trends in the dataset, but the Pearson correlation analysis clarified many important interspecific interactions on a finer scale. Although each correlation analysis only selected the top 10 taxa within each site, these taxa are likely playing a greater role in affecting endophyte community composition (Vandenkoornhuyse et al. 2015; Agler et al. 2016). The most salient trend was found when comparing the number of checkerboards within each site to the number of significant positive and negative interactions that could be leading to checkerboard colonization patterns. For example, the Coast Range site exhibited more significant interactions relative to the remaining three, and many of these interactions were negative when compared with the presence of OTU2. The site which possessed the second largest number of checkerboards, MSH control, also exhibited a greater number of significant interactions including negative interactions between OTU2 and others. In 
contrast, the Coast and MSH impacted sites had relatively few significant interactions and additionally had lower numbers of checkerboards. We acknowledge two potential shortcomings of this analysis when applied to a culture-independent study which leverages NGS technology. First, from a technical standpoint, there is a finite amount of space on the Illumina sequencing flow cell, and amplicons of DNA that are present in greater abundance will consume more of this space to the detriment of other amplicons. Still, the technology is highly sensitive to amplicons of DNA in very low abundance (Shokralla et al. 2015), and by converting the OTU table to an incidence matrix, biases in sequencing read numbers are accounted for. Second, from a biological perspective, the host tissue also represents a finite space for colonization, especially when considering that most endophytes form localized infections in their host (Rodriguez et al. 2009). This also would lead to the conclusion that abundant OTUs are negatively correlated with less abundant OTUs, as more of one taxon could mean less of another. Still the correlation analysis was able to clarify which OTUs were driving the checkerboard patterns observed, and further demonstrated that OTU2 appears to competitively exclude other taxa from the regional species pool (Chapter 2).

The taxonomic assignments of OTUs for this dataset clearly demonstrate the homogenous nature of endophyte communities in P. munitum across the region, particularly in the Coast Range and MSH control sites. Even more striking is how distinctive the neighboring plant endophytes are when growing in close proximity to the ferns. The Coast and MSH impacted sites are more similar in endophyte taxonomy within fern hosts as are the Coast Range and MSH control sites, even though these represent the 
most distant and second-most distant sites examined in the study, respectively. Several abiotic or ecological factors could be driving these results. First, although the Claussenomyces genus comprises a lower relative abundance in the Coast and MSH impacted sites, it is still present in these study sites; however, here it may be competitively inferior, allowing co-occurring taxa to increase in relative abundance. Second, more extreme abiotic conditions at the Coast and MSH could bolster more interspecific co-existence than is predicted at stable sites in equilibrium (Kennedy et al. 2014). Third, the impact of the 1980 eruption at Mount Saint Helens has created more habitat heterogeneity and more opportunities for diverse inhabitants (Che-castaldo et al. 2015), though richness and diversity estimates were not significantly greater at this site. Finally, prevailing trade winds moving from the Coast up the Columbia Gorge could place the Coast Range site and MSH control sites within dispersal ranges of fungal inoculum, while the Coast site and MSH impacted site is separated from the others by vicariance (the Coast Range and Western Cascades, respectively). Still, without directly testing these possibilities, we cannot reasonable adhere to any of the abovementioned predictions. Further, when considering neighboring plants, the MSH impacted and Coast sites were more similar in endophyte taxonomy from a coarse perspective, and this was also demonstrated in ordination. Extensive site metadata is not analyzed in the present work and predictions at this point are limited, but plant community composition was unique at each site. The most obvious similarity of the Coast and MSH sites was the presence of Poa spp., since both sites were full sun. Additionally, the P. munitum host population structure is unknown at this point, yet could be playing a role in the host 
specificity patterns observed. If abiotic factors including prevailing winds are leading to observed patterns of endophyte community similarity between sites, this could also be occurring for ferns, as their spores are dust-like and capable of dispersing great distances (Sessa et al. 2016). Future work will seek to examine the host population structure as DNA extracts are still preserved at $-80^{\circ} \mathrm{C}$.

A large proportion of the fern samples across all sites are colonized by the genus Claussenomyces, of which the dataset includes several distinct genotypes (confirmed by alignments with representative sequences for these OTUs). The Coast Range site is the same location studied in the temporal project (Chapter 2) and the representative sequences from both studies (OTU1 and OTU2, respectively) match identically and also match the ITS1 region of Catenospora polysticola identically (Chapter 3). When considering the potential source of inoculum as discussed in Chapter 2, all plants included in this study possessed senescent material around their base. If this is serving as an inoculum source, it may not be occurring as extensively in all sites, even though OTU2 is present in ferns from every site and absent from neighbors in every site. Still, there may be some abiotic factor resulting in these differences in colonization from senescent material if this is truly the initial source of fungal colonists.

\section{Conclusions}

The patterns of highly homogenous endophyte communities within a single host species across a 150-km range are unlike any studies present in the literature to the best of our knowledge. Further, higher similarities in endophyte community composition of distant hosts than in neighboring plants growing in close proximity is a factor that deserves more 
attention in other fern-endophyte systems. When considering the host tissue as endophyte "habitat," the patterns observed are potentially driven by strong filtering on behalf of the fern host, whereby co-occurring taxa are unable to colonize or are driven to competitive exclusion by OTU2. Additionally, distance decay is presumed to affect microbial distributions globally, yet we find little evidence of that at work in $P$. munitum endophyte communities. The present work has resulted in several additional questions which should be addressed in the near-term: (i) Is the underlying pattern in host-specificity of fern communities plant- or endophyte-derived (or both)? (ii) Is the abundant OTU examined in all three chapters of this work actually a plant mutualist? and (iii) What other plant systems demonstrate these patterns or is this a phenomenon restricted to $P$. munitum? We conclude that the likely source of fungal inoculum discussed in Chapter 2 -senescent material around the base of the host plant—could still explain the results observed in the present work since it was found in all P. munitum plants at each site. If confirmed in future work, it would be a special case of endophyte vertical transmission occurring through host leaf phenology (i.e. from old leaf to new leaf), allowing these taxa to thrive in a long-lived and stabile habitat created by their host. 


\section{Chapter 4}

Catenosporaceae fam. nov., to include Catenospora gen. nov.; fungal endophytes living in ferns and alder in the Pacific Northwest of North America

\section{Introduction}

The kingdom of fungi is a highly diverse group of eukaryotic microbes that occupy an impressive array of niches globally (Peay et al. 2016). Many questions regarding their evolutionary history and ecological function remain unanswered largely due to their diversity, cryptic manner of colonizing terrestrial habitats and difficulty of study under artificial conditions (Nilsson et al. 2014). Since plants initially colonized land through the facilitation of fungi (Winfred et al. 1994; Selosse and Le Tacon 1998), and continue to rely upon fungal associations for nutrient foraging (Brundrett 2009), pathogen defense (Arnold and Herre 2003), and the liberation of organic matter bound in recalcitrant plant compounds (Fernandez and Kennedy 2016), all while facing frequent antagonism from pathogenic fungi (Glazebrook 2005), it is likely that there are millions of undiscovered fungal taxa globally that colonize plants in some form (Hawksworth and Rossman 1997). One such group of fungi-endophytes - are symbiotrophic microbes that colonize intercellular spaces of hosts asymptomatically in all plant species studied and comprise a significant component of the plant microbiome (Arnold et al. 2009). They are described as hyperdiverse in the tropics (Arnold et al. 2000) and although many are likely commensals (May 2016), an emerging body of literature over the last decade has documented their ability to confer biotic and abiotic stress tolerance to their hosts, providing evidence that some may be plant mutualists (Busby et al. 2013, 2015a). Here, 
we propose Catenosporaceae fam. nov. and Catenospora gen. nov. within the Leotiomycetes which colonizes the foliar tissue of temperate ferns in the Polystichum Roth genus in Western North America. Additionally, another related taxon within the same fam. and gen. nov. has been isolated on several occasions from Alnus viridis ssp. sinuata (Regel) Löve \& Löve in a habitat in which it co-occurs with ferns of the Polystichum genus.

Although research into fungal endophytes has substantially progressed over the past two decades (Clay 1988; Redman et al. 2002; Arnold et al. 2003; Zimmerman and Vitousek 2012; Busby et al. 2015a), most of the work has focused on seed plant systems. In contrast, investigations of endophyte communities that colonize ferns have substantially lagged behind, though some culture-based work has documented the identity of several fern endophytes in temperate and tropical regions (Fisher 1996; Zubek et al. 2010; Del Olmo-Ruiz and Arnold 2014, 2017). However, we currently lack an understanding of how the diversity of fern endophyte communities ranks with other seed plant hosts, whether these fungal communities are more or less persistent in evergreen fern populations, and what functional role certain taxa play in stressful conditions. Recent work on the endophytes that colonize the temperate fern host, Polystichum munitum, has uncovered the presence of a dynamic fungal community that is dominated by one of the taxa described in this work (Younginger and Ballhorn 2017). However, the diversity of this fern endophyte community is substantially lower than typically found in other seedreproducing hosts uncovered in culture-independent work (Zimmerman and Vitousek 2012; Bálint et al. 2013, 2015; Busby et al. 2015a). It is currently unclear if this is an 
effect of fern host manipulation of its microbiota or the result of superior competition or repeated dispersal by this taxon (Smith et al. 2018).

The taxa described in this work are most closely related to ecologically heterogeneous groups of fungi within the order Helotiales based on molecular data. The Helotiales is the largest order within the Leotiomycetes (Ascomycota) and contains 12 families, 395 genera (92 of which remain unclassified) and 4,000 species (Wang et al. 2006a; Suija et al. 2014). Teleomorphs possess apothecia with unitunicate, inoperculate asci that may be undiagnostic for the group, though many are only known as anamorphic (Baschien et al. 2006, 2013). Members of the Helotiales include aquatic hyphomycetes (Seena and Monroy 2016), endophytes (Wang et al. 2006b), mycorrhizae-formers, pathogens, saprobes, and fungicolous fungi (Suija et al. 2014). The most closely-related taxa to the fam. nov. described herein include members of the genus Collophora Damm and Crous (which are pathogens of woody Prunus tissue; Damm et al. 2010), Claussenomyces Kirschst (a colonist of conifer resin exudates; Hawksworth \& Sherwood 1981) and Gelatinomyces Sanoamuang, Jitjak, Rodtong \& Whalley (a pathogen of bamboo; (Sanoamuang et al. 2013). Two of the abovementioned genera (Collophora and Gelatinomyces) are currently Leotiomycetes in. sed., while Claussenomyces is placed within Helotiaceae - a more distant family. Since many current taxonomic placements within the Helotiales and even the Leotiomycetes have relied upon conidia morphology, the application of molecular methods to the phylogenetics of this group has revealed that former classifications are largely polyphyletic (Wang et al. 2006a). Clearly more work is 
deserved in this large and heterogenous group of fungi for a formal understanding of evolutionary relationships between extant taxa.

We propose four new species within Catenosporaceae fam. nov., Catenospora gen. nov. that were isolated on several occasions from healthy, surface sterilized leaflets of western swordfern, Polystichum munitum (Kaulf.) C. Presl and narrowleaf swordfern, Polystichum imbricans (Eaton) Wagner, and also from surface sterilized leaf tissue of sitka alder, Alnus viridis ssp. sinuata. These anamorphic taxa are described through morphological characters and multigene phylogenies of the internal transcribed spacer (ITS), the 28S large subunit (LSU) of rDNA, and the RNA polymerase subunits I and II (RPB1 and RPB2). Both morphological and molecular analyses support the placement of these taxa within a new family and genus, with uncertain placement in an order based on current taxonomic circumscriptions (i.e. Leotiomycetes in. sed.). In addition to our isolation of these fungi on artificial media, we have discovered a high extent of colonization from one species over an entire growing season from a population of $P$. munitum in the Oregon Coast Range utilizing culture-independent techniques. This increase in colonization, with a concomitant reduction in competing endophytes in the community, suggests a functional or ecological importance of this taxon that has yet to be clarified.

Methods

Study sites, collections, and hosts-

Two of the taxa described herein-C. polysticola and C. gilkeyae — were isolated from surface sterilized foliar tissue of the temperate fern, Polystichum munitum 
(Dryopteridaceae), in the eastern foothills of the Oregon Coast Range at 45.733759 N, 123.18631 W and 310 meters above sea level from three different plants on 8/8/2014 and from four different plants between 4/26-9/9/2014, respectively. The remaining two taxa were isolated from surface sterilized foliar tissue of $P$. imbricans (C. carrollii) and $A$. viridis ssp. sinuata (C. loowitii) at the base of Mount Saint Helens at approximately 46.230447 N, 122.156997 W and 1283 meters above sea level from two different plants on 7/28/2015 and from two different plants on 10/13/2014, respectively. Healthy foliar tissue — showing no visible signs of lesions or herbivore damage — were isolated with pruning shears dipped in 95\% ethanol and wiped clean with a fresh Kimwipe (Roswell, GA, USA). Leaves (from Alnus) or leaflets (from Polystichum) were placed in clean, unused coin envelopes and transported to the lab within six hours of sampling. Plant tissue was refrigerated until surface sterilization and plating on culture medium. All surface sterilization and plating was performed within 24 hours of returning from the field. Briefly, leaf tissue was held under running tap water for $30 \mathrm{~s}$ to dislodge epiphytic microbes and then submerged in sequential baths of $95 \%$ ethanol, $0.5 \% \mathrm{NaOCl}$, and $70 \%$ ethanol (10 sec, $2 \mathrm{~min}, 2 \mathrm{~min}$, respectively) to sterilize the surface and then were allowed to air dry on a clean Kimwipe in a hood (Arnold et al. 2003). After all ethanol evaporated from the surface (ca. $2 \mathrm{~min}$ ), leaflets were cut into $2 \mathrm{~mm}$ x $2 \mathrm{~mm}$ segments with a sterilized scalpel and plated onto malt extract agar (MEA; $2 \% \mathrm{w} / \mathrm{v})$. Plates were stored at both room temperature $\left(23^{\circ} \mathrm{C}\right)$ in ambient indoor lighting conditions and observed daily for signs of visible fungal growth. Following seven days of initial culturing, any visible hyphae growing out of the leaf surface were re-isolated into new MEA dishes, making 
axenic cultures. Axenic cultures were given a unique accession number and served as the source for all subsequent re-isolations and molecular/microscopic analyses. Morphology and Microscopy-

For macroscopic observation of growth, three, $5 \mathrm{~mm}$ punches were taken from axenic source cultures and plated onto MEA and potato dextrose agar (PDA; $2 \% \mathrm{w} / \mathrm{v})$. These plates were stored at $23^{\circ} \mathrm{C}$ for two weeks prior to imaging. Cultures were placed on a stand with a camera mount and imaged with a macro lens on a digital single-lens reflex camera.

For microscopic observation, slides were prepared from both MEA culture dishes (scraped with a sterile scalpel) and from inoculated Triticum grain. The Triticum grain was rinsed in running tap water and transferred to clean $30 \mathrm{~mL}$ McCartney bottles and autoclaved at $121^{\circ} \mathrm{C}$ for one hour at $3 \mathrm{~atm}$ of pressure. After cooling overnight, bottles were inoculated with $5 \mathrm{~mm}$ punches of each culture and incubated in ambient conditions $\left(23^{\circ} \mathrm{C}\right.$ and 12 hours of daily ambient indoor light) for three months. Individual grains were removed from the McCartney bottles with sterile forceps and scraped onto clean slides with a drop of glycerol solution $(50 \% \mathrm{v} / \mathrm{v})$. Images of hyphal growth and conidia were generated on a Leica confocal microscope with differential interference contrast settings (441 nm laser) and scale bars were added with Leica software.

Molecular-

One $\mathrm{mm}^{2}$ pieces of hyphae were isolated with autoclaved toothpicks and fungal DNA was extracted with the REDExtract-N-Amp kit (Sigma-Aldrich; Darmstadt, Germany). DNA products were amplified with PCR using the Sigma GoTaq mastermix (Sigma-Aldrich; 
Darmstadt, Germany) with primers and cycling parameters according to Appendix A. Successful PCR was verified with agarose gel electrophoresis. Gel excision was performed for any PCR reactions that yielded multiple products. Gel-excised and raw PCR products were cleaned and sequenced with Sanger technology at Functional Biosciences (Madison, WI, USA). Forward and reverse AB1 files from sequencing were combined using Geneious 10.0.5 (Kearse et al. 2012) and sequences were initially examined using the BLAST algorithm against the NCBI and UNITE (Kõljalg et al. 2013) databases. PhylogeneticsAdditional fungal sequences from members of the Leotiomycetes and the Geoglossomycetes (as an outgroup) were obtained from NCBI, utilizing the previous work of several groups (Appendices B \& C). Two separate phylogenetic analyses were conducted: a narrow analysis that examined the most closely-related taxa to the clade proposed herein (ITS-LSU; 26 additional taxa) and a wide analysis that examined more distant relationships (ITS-LSU-RPB1-RPB2; 90 additional taxa). The narrow analysis utilized sequence data from the entire ITS region (intergenic transcribed spacer; ITS1, 5.8S, ITS2) and the LSU region (large subunit; 28S). Since the ITS region of rDNA is difficult to align for fungi and both the ITS and LSU regions are less robust to phylogenetic analyses (Bruns 2001; Vellinga et al. 2015), we relied upon sequence data from these regions because they were the only data available for the most closely-related taxa. The wide analysis incorporated the ITS and LSU regions and additionally included 
sequence data from two largest subunits of RNA polymerase II (RPB1 and RPB2; Matheny et al. 2002).

Sequences were aligned with MAFFT 1.3.7 implemented in Geneious 10.2.3 using automatic algorithm selection. Alignments were manually trimmed and adjusted as necessary in Geneious 10.0.5, realigned with Muscle 3.8.31 and manually adjusted again where necessary. For the RPB regions, alignments were edited to remove introns (two in RPB1 and one in RPB2) to ensure reading frame fidelity among the sequences and were realigned by translation with Muscle. Alignments for both analyses were processed with TrimAl 1.2 (Capella-Gutiérrez et al. 2009) using the automated1 (for ITS and LSU) and the gappyout (for RPB1 and RPB2) arguments.

Both maximum likelihood (ML) and Bayesian methods were used to analyze the phylogenetic results of alignments. The best model of evolution for each region (narrow: ITS1, 5.8S, ITS2, LSU and wide: ITS1, 5.8S, ITS2, LSU, RPB1, RPB2) was determined with PartitionFinder 2.1.1 (Lanfear et al. 2012) based on the lowest value of AICc. From these results, the alignments were split into partitions (narrow: 1) ITS1 and ITS2 2) 5.8S and LSU; wide: 1) ITS1 and ITS2 2) 5.8S and LSU 3) codons 1 and 2 of RPB1 and RPB2 4) codon 3 of RPB1 and RPB2). The ML analysis was performed with RAxML 8.2.11 (Stamatakis 2014) following a general time reversible model (GTR) of substitution assuming a gamma distribution and a proportion of invariable sites $(\mathrm{GTR}+\mathrm{I}+\mathrm{G})$ for the narrow analysis and without a proportion of invariable sites $(\mathrm{GTR}+\mathrm{G})$ for the wide analysis with 500 bootstrapping replicates and the resulting bipartition data were written to the best-scoring ML trees following a 50-tree search. 
The Bayesian analysis was conducted with BEAST 1.8.4 (Drummond et al. 2012) with the same partitions and models of evolution as in the ML analysis. Substitution models and clock models were unlinked for each partition while trees were linked. For both the narrow and wide analysis, strict clock models were used for each partition with a Yule speciation process tree prior (Gernhard 2008) employed for the tree model.

Remaining priors were left with default settings. A 10,000,000 state MCMC run was initiated with the best-scoring ML tree as a starting tree and sampled at every 1000 states. Resulting trace files with were examined with Tracer 1.6.0 (http://tree.bio.ed.ac.uk/software/tracer/) to ensure convergence of the posterior distribution and a reasonable effective sample size (ESS > 200). Posterior probabilities were written to a maximum clade credibility target tree (narrow analysis) and the bestscoring ML tree (wide analysis) with TreeAnnotator 1.8 .4 with a 5\% burn-in. Trees were annotated in FigTree 1.4.3 (http://tree.bio.ed.ac.uk/software/figtree/) and ML bootstrap values were added to the posterior probabilities. Bootstrap values $>70$ and posterior probabilities $>0.95$ were deemed significant.

Results

Both the wide and narrow phylogenetic analyses show strong support for the placement of the taxa described in the present work within a fam. nov. (Catenosporaceae) and gen. nov. (Catenospora) based on current taxonomic circumscriptions of related groups. The narrow analysis combined 30 taxa and 1029 sites with 459 variable and 570 constant. Following both ML and Bayesian frameworks, the taxa described herein form a separate group most closely-related to the genera Collophora, Gelatinomyces (Leotiomycetes in. 
sed.) and Claussenomyces (Helotiaceae). Further, Catenosporaceae appears to be sister to

\section{Cyclaneusma and Naemacyclus (Helotiales in. sed.) and Marthamyces (Rhytismataceae)}

and more distantly related to the Microglossum and Leotia clade (Leotiomycetes in. sed.

and Leotiaceae, respectively; Figure 4.1).

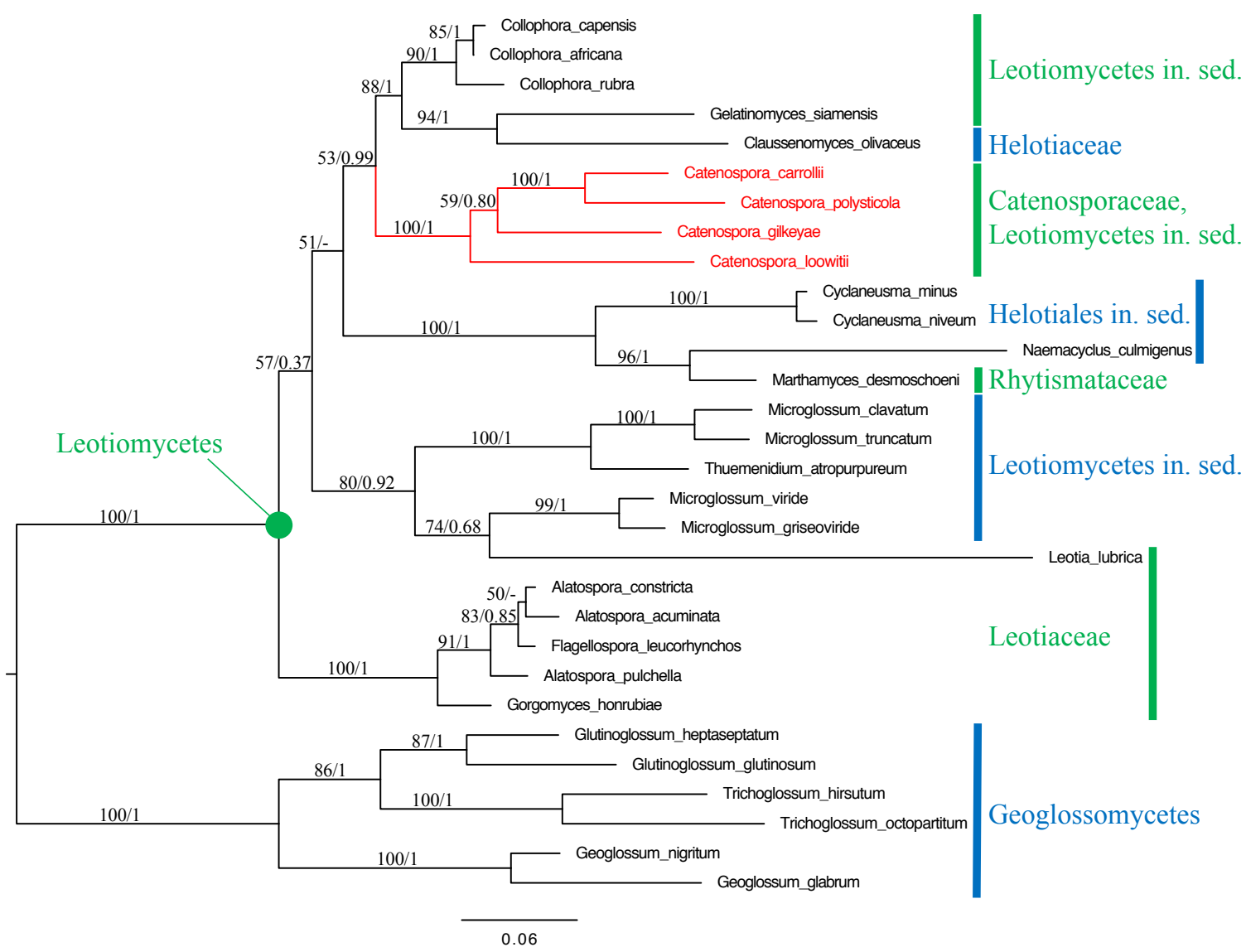

Figure 4.1 Phylogenetic tree from the narrow analysis showing the relationship of Catenosporaceae to other, closely-related Leotiomycetes. Tree topology generated from combined maximum likelihood (ML) and Bayesian analyses of ITS and LSU regions. The ML method utilized 500 bootstraps followed by $50 \mathrm{ML}$ tree searches. The best tree from the ML analysis served as a starting tree for 10,000,000 MCMC states with logging at every 1000 states. Posterior probabilities from the MCMC run were written to the maximum clade credibility tree with a 500,000-state burn in. Bootstrap values and posterior probabilities are placed above branches, respectively, and branches in disagreement between ML and Bayesian methods contain only bootstrap values (i.e. 100/-). Scale bar represents the proportion of substitutions per site over a $1029 \mathrm{bp}$ alignment. Catenosporaceae fam. nov. is highlighted in red and green/blue annotations with corresponding taxonomic classifications are arbitrarily selected for easier viewing. 
The wide analysis combined 94 taxa and 2968 sites with 2090 variable and 878 constant. Significant branch support confirms that Catenosporaceae is, again, sister to Microglossum and Leotia and more distantly-related to a diverse grouping of taxa (Figure 4.2). Additionally, both the narrow and wide analysis agree with more recent ancestry between C. polysticola and C. carrollii with C. loowitii as more distantly related.

\section{Taxonomy}

The endophytes isolated from surface sterilized fern and alder foliar tissue are morphologically and genetically distinct from known taxa in molecular databases. The new family Catenosporaceae and new genus Catenospora is proposed and comprised of the four taxa described herein, forming a monophyletic group. Catenosporaceae, Younginger \& Stewart, fam. nov. Teleomorphs. unknown.

Type genus. Catenospora Younginger \& Stewart, gen. nov. Type species. Catenospora polysticola Younginger \& Stewart, sp. nov. Etymology - Possesses spherical conidia that form unbranching chains (caten- L. $=\mathrm{a}$ chain).

Anamorphic Ascomycetes with septate, perpendicularly branching hyphae (5-10 $\mu \mathrm{m}$ wide and 10-20 $\mu \mathrm{m}$ long), or yeast stages (5-15 $\mu \mathrm{m}$ wide) and possessing micronematous, catenulate thalloconidia or endoconidia that are spherical to ovate, 5-15 $\mu \mathrm{m}$ long on sterilized Triticum grain (Figure 4.3). Colonies produce dense, aerial hyphae on artificial media (MEA and PDA) with dematiaceous hyphae at the center and downy hyphae protruding at the periphery or pink glabrous colonies with aerial growth in yeast stages 


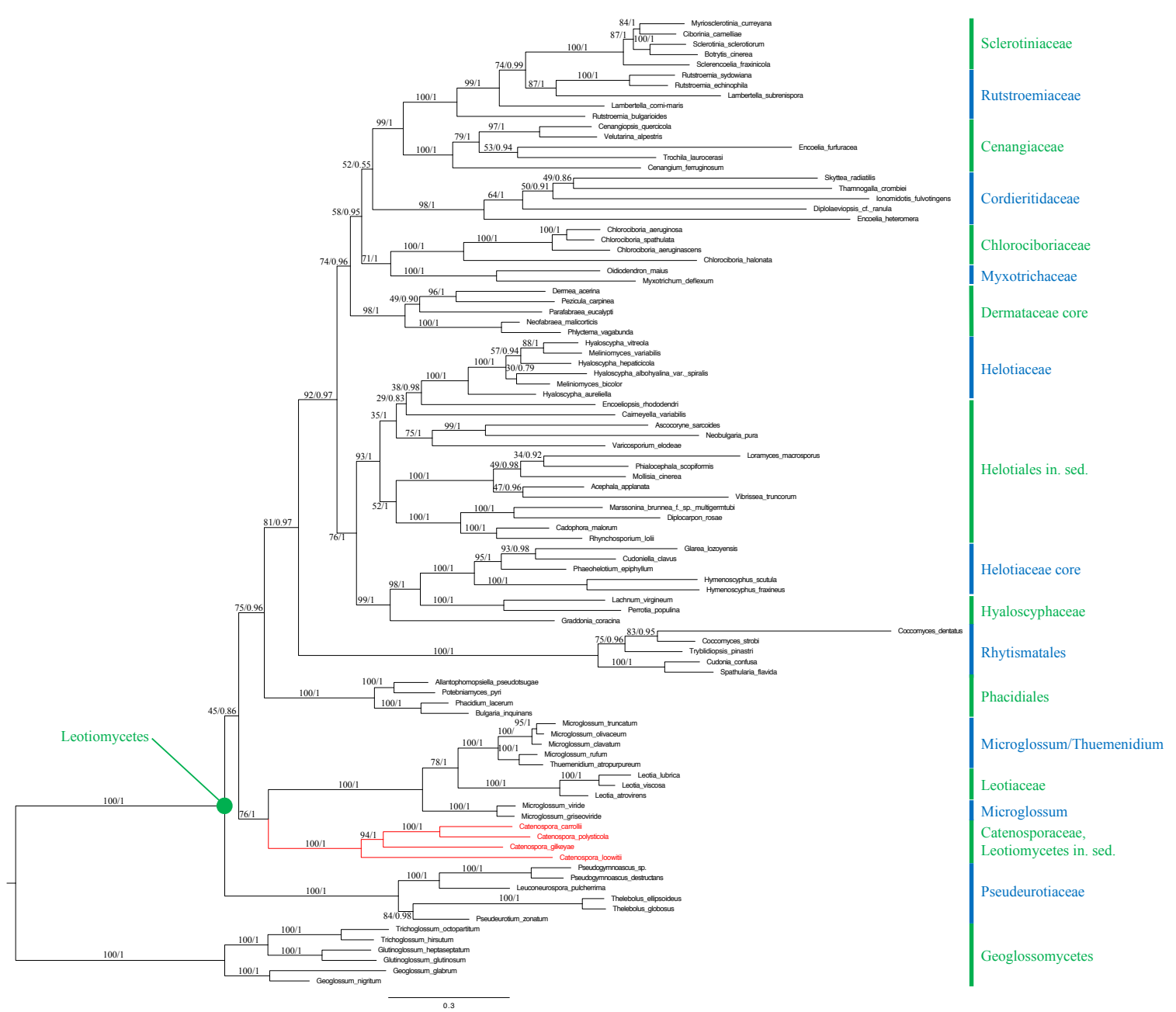

Figure 4.2 Phylogenetic tree from the wide analysis showing the relationship of Catenosporaceae to other, closely-related Leotiomycetes. Tree topology generated from combined maximum likelihood (ML) and Bayesian analyses of ITS, LSU, RPB1, and RPB2 regions. The ML method utilized 500 bootstraps followed by $50 \mathrm{ML}$ tree searches. The best tree from the ML analysis served as a starting tree for $10,000,000$ MCMC states with logging at every 1000 states. Posterior probabilities from the MCMC run were written to the best-scoring ML tree with a 500,000-state burn in. Bootstrap values and posterior probabilities are placed above branches, respectively. Scale bar represents the proportion of substitutions per site over a $1029 \mathrm{bp}$ alignment. Catenosporaceae fam. nov. is highlighted in red and green/blue annotations with corresponding taxonomic classifications are arbitrarily selected for easier viewing. 
(particularly in PDA; Figure 4.4). Colonies slow-growing on MEA and PDA at $22^{\circ} \mathrm{C}$ and $4^{\circ} \mathrm{C}$ in both dark conditions and with ambient indoor light.Notes - The taxa described are differentiated from the closely-related Claussenomyces Kirschst. which is known to produce sessile dark green to black ascomata with pigment localized to the peripheral hyphae, though hyphae become coriaceous when dry in a similar manner to Catenospora polysticola (Hawksworth and Sherwood 1981). The conidia of Claussenomyces are similarly enteroblastic, cylindrical and hyaline as in Catenospora carrollii, but condiogenous cells with a small collarette are present and conidia are not catenulate. Gelatinomyces Sanoamuang, Jitjak, Rodtong \& Whalley is known to produce large ascostromata that are dark in color on bamboo with colonies that are white and powdery leaving a dark red pigment in the medium and lacking aerial hyphae (Sanoamuang et al. 2013). They do possess ovoid conidia similarly to C. carrollii; however, they are produced on both short or long conidiogenous cells. Another closely related genus, Collophora Damm \& Crous, produces moist, cream or reddish colonies with aerial mycelia occurring infrequently and short adelophialides or collarettes on the hyphae (Damm et al. 2010). Cyclaneusma (Butin) DiGosmo, Peredo and Minter produces apothecia on senescent conifer needles with 8-spored asci and filiform, hyaline ascospores that are smooth and doubly-septate (Dicosmo et al. 1983). Marthamyces Minter possesses white, dense hyphae surrounding ascomata and develops on fallen leaves without producing bleached zones and has filiform ascospores that are aseptate to singly septate (Minter 2003; Johnston 2006). The more distantly-related Microglossum (Pers.) Gillet clade, known as the green earth tongues, produces green ascocarps with 
green to green-yellow ascomata and is commonly associated with spruce, beech and oak forests (Kučera et al. 2014) while members of the genus Leotia (Scop.) Pers. produce ochre to olive-green ascocarps (commonly called jelly babies) and are often found growing with moss and decaying plant material (Verkley 1994). Finally, the genera Alatospora Marvanoá \& Descals and Flagellospora (Ingold) Petersen—a group of aquatic fungi-are contrasted from Catenosporaceae due to a lack of phialides. Although species within Alatospora and Flagellospora possess polymorphic conidia, they often produce branched, stauroform or scolecoform conidia (Petersen 1963; Marvanová and Descals 1985; Jooste and Merwe 1990), which is not observed with the type specimens. Additionally, colonies of Alatospora grow rapidly and are glaborous (Marvanová and Descals 1985). The genus Gorgomyces Gönczöl \& Révay, closely related to Alatospora and Flagellospora, is characterized as having filiform conidia with an apical walled appendage that aids in attachment to nematodes and a basal stalk cell that undergoes septation (Gonczol and Revay 1985; Roldan 1989). Further, conidia of Gorgomyces tend to dissociate together, forming radiating clusters of conidia.

Catenospora polysticola Younginger \& Stewart, sp. nov.

Figures $4.3 \mathrm{~A}, 4.3 \mathrm{E}, 4.4 \mathrm{~A}, 4.4 \mathrm{E}$

Etymology - Found growing within Polystichum leaves (-cola Lat. = dwell). Isolated as an endophyte from surface sterilized leaflets from Polystichum munitum (Kaulf.) C. Presl at 45.733759 N, $123.18631 \mathrm{~W}$ and 310 meters above sea level. Its distribution appears common across the region in P. munitum hosts along with another closely related taxon described in this work (C. gilkeyae). Colonies metachroic, white 
turning dematiaceous at the periphery and with age, dense aerial hyphae that are filamentous, often forming concentric rings of melanized and white hyphae in MEA. On PDA, colonies dense and dematiaceous, forming lobes with uneven margins and filamentous at the periphery. Often colonizes new regions of culture medium via conidial dispersal. Hyphae dark, septate, perpendicularly branching, 10-15 $\mu \mathrm{m}$ long and 2.5-5.0 $\mu \mathrm{m}$ wide. Lacking conidiogenous cells, instead possessing spherical to ovate thalloconidia 5-10 $\mu \mathrm{m}$ wide on Triticum grain.

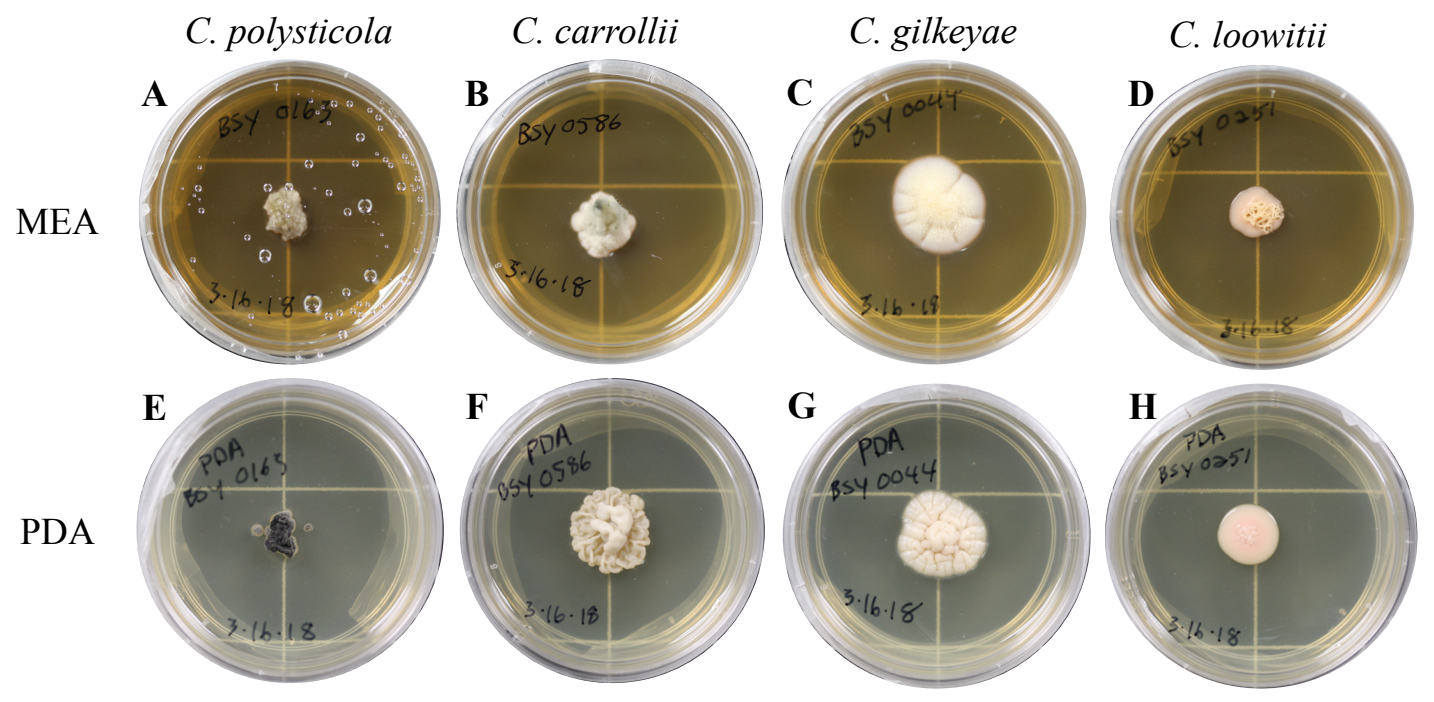

Figure 4.3 Colony characteristics of the four spp. nov. on malt extract agar (MEA) and potato dextrose agar (PDA). Columns represent individual taxa and rows represent the medium on which they were grown. Images generated with a digital single-lens reflex camera with a macro lens.

Notes - This species is contrasted with others in the genus by its highly dematiaceous colonies in culture with more irregular margins and concentric rings of pigmentation often occurring. The hyphae are more melanized than others with septa more obvious. The thalloconida are more apparent than other members of the genus on Triticum grain. 
Catenospora gilkeyae Younginger \& Stewart, sp. nov.

Figures $4.3 \mathrm{~B}, 4.3 \mathrm{~F}, 4.4 \mathrm{~B}, 4.4 \mathrm{~F}$

Etymology - Personal name for Helen Gilkey, pioneering mycologist and botanist

Ecology/isolation

Isolated as an endophyte from surface sterilized leaflets from Polystichum munitum

(Kaulf.) C. Presl at 45.733759 N, $123.18631 \mathrm{~W}$ and 310 meters above sea level. This

taxon was isolated at a single study site from four different host plants over 6 months. In

culture, basal hyphae mucoid, filamentous at the periphery, pink, corrugated, aerial

C. polysticola
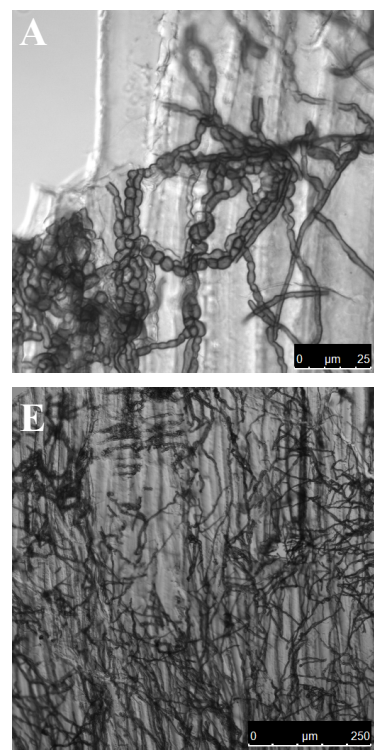

C. carrollii
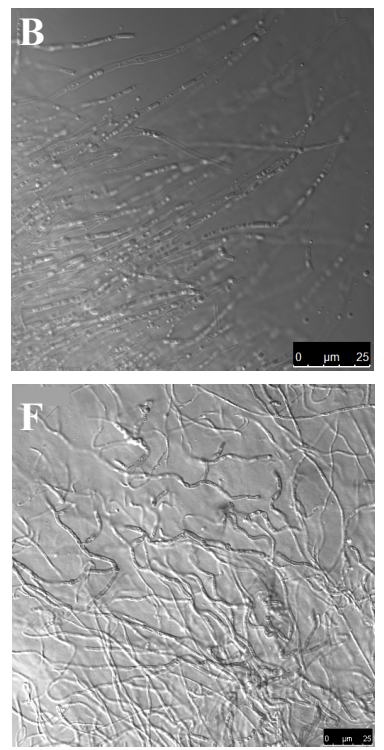

C. gilkeyae
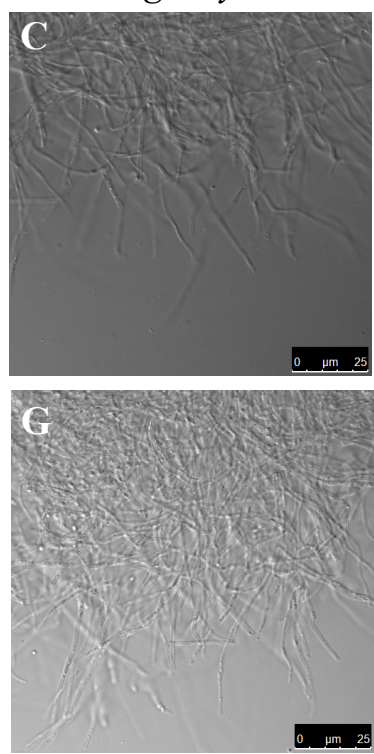

C. loowitii
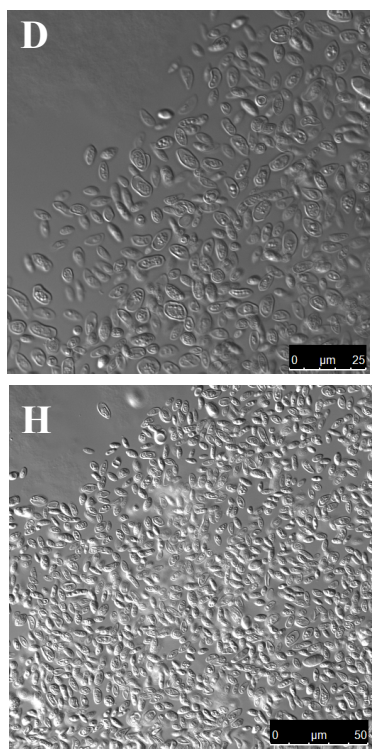

Figure 4.4 DIC images of hypha and conidium morphology of the four spp. nov. on malt extract agar (MEA; C. loowitii) or Triticum grain (all others). Columns represent individual taxa and rows represent replicates of individual taxa. Images were generated with a Leica TCS SP8 confocal microscope at $441 \mathrm{~nm}$ and scale bars were added with Leica software.

hyphae with even margins on MEA. In PDA, highly mucoid and yeast-like with occasional filamentous protrusions, more corrugated than in MEA, light pink with even margins. Hyphae septate, hyaline, infrequently branching, 10-15 $\mu \mathrm{m}$ long, 2.5-5.0 $\mu \mathrm{m}$ 
wide. Conidiogenous cells absent, endoconidia forming within hyphae, spherical, $5 \mu \mathrm{m}$ wide.

Notes - C. gilkeyae is downier in appearance on MEA than other members of the genus with even, regular corrugation in both MEA and PDA. Hyphae are more hyaline than in C. polysticola and septa are less apparent. Hyphae branch less frequently, though more so than C. carrollii. Conidia are less apparent on Triticum grain, forming endoconidia rather than catenulate thalloconidia.

Catenospora loowitii Younginger \& Stewart, sp. nov.

Figures $4.3 \mathrm{C}, 4.3 \mathrm{G}, 4.4 \mathrm{C}, 4.4 \mathrm{G}$

Etymology - The indigenous name for Mount Saint Helens (Loowit), where this taxon was isolated.

Ecology/isolation

Isolated from surface sterilized foliar tissue of $A$. viridis ssp. sinuata at the base of Mount Saint Helens at approximately 46.230447 N, $122.156997 \mathrm{~W}$ and 1283 meters above sea level. Distribution may be restricted as no other isolates were obtained away from the primary sites described, though sampling was intensive. In culture, highly mucoid at the base with serpentine aerial hyphae and even margins; bright pink in MEA. In PDA, highly mucoid or liquid in appearance, bright pink, with fewer serpentine projections, very even margins. Filamentous forms absent on MEA, PDA, and Triticum grains. Yeast form ovate, 10-15 $\mu \mathrm{m}$ long and 5-10 $\mu \mathrm{m}$ wide, infrequently catenulate. Conidia absent, though dividing cells apparent. 
Notes - This species lacks filamentous forms on MEA, PDA, and Triticum grain and is more mucoid and pink in color. Yeast cells are ovate and hyaline and infrequently catenulate. Conidia are absent in all culture methods tested.

Catenospora carrollii Younginger \& Stewart, sp. nov.

Figures $4.3 \mathrm{D}, 4.3 \mathrm{H}, 4.4 \mathrm{D}, 4.4 \mathrm{H}$

Etymology - Personal name for George Carroll, distinguished mycologist who conducted seminal research on symbiotrophic microfungi

Ecology/isolation

Isolated from surface sterilized foliar tissue of $P$. imbricans at the base of Mount Saint Helens at approximately 46.230447 N, $122.156997 \mathrm{~W}$ and 1283 meters above sea level from two different plants on 7/28/2015. In culture, metachroic with basal portions pink, dematiaceous at the periphery, dense and filamentous, aerial, occasional corrugation with undulating margins on MEA. In PDA, white to pink, highly lobed with undulating projections, glaborous, undulating margins and yeast-like in appearance. Hyphae hyaline, septate, serpentine and obtusely branching, $10-15 \mu \mathrm{m}$ long and 2.5-5.0 $\mu \mathrm{m}$ wide. Conidiogenous cells absent, forming cylindrical endoconidia 7-10 $\mu \mathrm{m}$ long and 2-4 $\mu \mathrm{m}$ wide or terminal spherical to ovate conidia 5-10 $\mu \mathrm{m}$ wide.

Notes - Most similar in appearance to C. polysticola on MEA, this species possesses more pronounced undulating projections than others on PDA. Hyphae branch less frequently than both C. polysticola and C. gilkeyae and septa are less apparent as in C. gilkeyae. The cylindrical endoconidia of this species are contrasted with the highly apparent thalloconida of C. polysticola. 


\section{Discussion}

Both morphological and molecular results strongly suggest the discovery of a novel monophyletic group of taxa, living as endophytes in the Pacific Northwest of North America. Their colony characteristics, hyphal growth, and conidial morphology is unique when compared to descriptions of the sister genera Claussenomyces, Gelatinomyces and Collophora (Hawksworth and Sherwood 1981; Damm et al. 2010; Sanoamuang et al. 2013). Specifically, they maintain dense, aerial tuberculate mycelia with undulating margins and gray, villose peripheral hyphae or pink mucoid yeast forms with slow growth rates at both $4^{\circ}$ and $22^{\circ} \mathrm{C}$ in dark and light conditions. Additionally, most members possess spherical to ovioid catenulate conidia without clearly defined conidiophores or phialides. We propose the addition of the four Catenospora spp. nov. to the Catenosporaceae fam. nov.

Our narrow phylogenetic reconstruction of the ITS-LSU alignment is in agreement with the recent work by Baschien et al. (2013) showing a polyphyletic grouping of Alatospora, Flagellospora, and Gorgomyces, though several of their statistical relationships remain unresolved. Still, although we included several taxa that are more closely related within the Leotia clade than in the abovementioned work, their close grouping of Microglossum and Leotia is in agreement with our results in both the narrow and wide analysis and also in agreement with Wang et al. (2006a,b). One key difference from the narrow analysis is seen in an examination of the Rhytismatales which places Marthamyces, Cyclaneusma, and Naemacyclus as more distantly related to Leotia (Lantz et al. 2011), yet we demonstrate that the three genera are sister to our clade and 
more closely related to Leotia, though their analysis relied upon the mitochondrial small subunit (SSU) in addition to the LSU of rDNA.

The wide reconstruction of the ITS-LSU-RPB1-RPB2 alignment shows many agreements with the work of others. Specifically, the placement of Bulgaria of the Phacidiales as sister to the Leotia/Microglossum clade, the placement of the Rhytismatales (including Cudonia and Spathularia) as sister to the Phacidiales/Leotiaceae s.s., the close relationship of Vibrissea (Vibrisseaceae) and Mollisia (Dermateaceae) to Hyaloscyphaceae and Helotiaceae, that Neofabraeae and Dermea fall within the core Dermataceae and Hyaloscypha (Hyaloscyphaceae) is sister to this group, the placement of Lachnum as sister to Hymenoscyphus and Cudoniella (core Helotiaceae), and the close relationship of Ascocoryne to Neobulgaria have all been confirmed independently by others (Wang et al. 2006a, b; Lantz et al. 2011; Baschien et al. 2013; Johnston et al. 2014). In contrast, Baschien et. al. (2013) place Cadophora as distant from Cudoniella and Hymenoscyphus and close to Hyaloscypha, yet our wide results show Cadophora as more closely-related to Helotiaceae than to Hyaloscypha (Hyaloscyphaceae). Additionally, the lack of more exclusive taxonomic assignments seen in the middle of our wide analysis (between the Rhytismatales and Dermataceae), demonstrate the critical need for further circumscription of these relationships within the Leotiomycetes.

While members of Catenosporaceae fam. nov. may have an affinity with the genera Collophora, Claussenomyces and Gelatinomyces, the taxa proposed in the present work are monophyletic based on morphological differences and phylogenetic analysis. 
Rather than attempt to combine our group with the Collophora clade or Gelatinomyces (both currently Leotiomycetes in. sed.), or with Claussenomyces (currently in Helotiaceae, a large family that is distantly related and polyphyletic), it is most reasonable to define this morphologically and genetically distinct group as a fam. nov. with the recommendation that future circumscriptions of the Leotiomycetes s.l. combine the aforementioned genera into Catenosporaceae if deemed appropriate based on additional collections and analyses. Further, as surveys of other Polystichum populations will continue in North America by our group, it is likely that more species will be added to Catenosporaceae fam. nov. in the future.

One of the fungal endophytes described herein (C. polysticola) was isolated on several occasions from a population of $P$. munitum hosts in the eastern foothills of the Oregon Coast Range as part of a separate study examining the temporal turnover of microfungi in ferns (Younginger and Ballhorn 2017). A monthly survey of fungal community turnover from an entire growing season of the host plant using Illumina technology suggests that this taxon is abundant, highly competitive or a superior colonist (Smith et al. 2018), and ecologically important. However, the relatively short amplicons generated through Illumina sequencing are not phylogenetically informative for fungi (Bálint et al. 2014), and the clustering of NGS sequencing reads to generate operational taxonomic units (OTUs) may have grouped together several taxa that are closely-related to two of the isolates described in this work (C. polysticola and C. gilkeyae). Still, it is likely that the strong signal detected in the culture-independent study-with a single OTU found in all plants and a concomitant reduction in co-occurring taxa-was due to 
the presence of C. polysticola, as the short amplicon reads from the Illumina platform match the ITS region of this isolate over its entire $279 \mathrm{bp}$ length. Additionally, there is a $69 \%$ difference between the OTU and the ITS region of $C$. gilkeyae over $279 \mathrm{bp}$, leading us to conclude that this taxon was not responsible for results found in the cultureindependent study. It is also notable that of 20 plants examined in the field study mentioned here, only two lost a single leaf (i.e. frond) throughout the entire survey—one to mammalian herbivory and one to pathogen-related senescence. Therefore, it is reasonable to suggest that $C$. polysticola is, at minimum, a commensal within the host and potentially a mutualist based on its impressive colonization ability which may be facilitated by numerous factors, including the host plant. It is currently unclear why this fungus is so abundant and persistent throughout multiple seasons, but a similar result was found by Sati and Belwal (2005b) when analyzing endophytes of the roots of pteridophytes in riparian habitats. Also, the initial source of colonizing inoculum is currently unknown and could include systemic growth from within the host, senescent material around the host plant, soil, or canopy throughfall (Del Olmo-Ruiz and Arnold 2014). Clearly, this fungus and closely-related taxa deserve more attention regarding their taxonomy, biology, and interesting ecology.

As investigations continue to uncover ecologically-important fungi from the hyperdiverse Helotiales in terrestrial and aquatic habitats, taxonomic clarifications become increasingly important (Wang et al. 2006a; Baschien et al. 2013; Johnston et al. 2014). Identifying shared traits amongst closely related taxa in a diverse fungal order will enable more robust predictions about the functional importance of other yet-to-be- 
described taxa. For example, although phylogenetic analysis supports our placement of Catenosporaceae near fungi that exist as saprotrophs (e.g. Microglossum and Leotia), pathogens (e.g. Collophora and Gelatinomyces) and aquatic hyphomycetes (e.g Flagellospora and Alatospora), both niche and guild lability are common within the Helotiales (Wang et al. 2006a). It is currently unclear if the endophyte in this work is conforming to the foraging ascomycete hypothesis (McMullin et al. 2018), waiting for host plant senescence to begin a saprobic lifestyle. Furthermore, it is unknown if other closely-related, regionally-specific taxa are found in ferns or other host plants in the Pacific Northwest. The importance of examining eukaryotic microbial communities in non-traditional host plant lineages cannot be overstated, since many undescribed taxa with interesting ecological functions will continue to be uncovered by inquiring researchers. (White et al. 1990; Matheny et al. 2002; O’Donnell et al. 2007) 


\section{Chapter 5}

Discussion

This comprehensive study of fungal endophyte communities in the temperate fern host, Polystichum munitum, demonstrates the importance of conducting microbial analyses in poorly understood plant systems. Through extensive culture-independent and -dependent sampling efforts, we were able to reveal previously unreported endophyte community dynamics with a dramatic reduction in diversity over the course of a growing season. The most abundant endophyte in the temporal project (Chapter 2) was found to be an inferior competitor in vitro yet existed as a commensal on gametophytes of the host plant. Additionally, we were able to clearly demonstrate a high degree of host specificity for fern endophytes across four distinct habitat types spanning a $150 \mathrm{~km}$ gradient (Chapter 3) with significantly segregating microbial communities dependent upon the host plant species. Although a distance decay in community similarity is predicted in fungal communities (Meiser et al. 2014), we did not find evidence of this occurring in $P$. munitum communities at the scale under study. Lastly, in the process of conducting the abovementioned culture-independent surveys, we have discovered that the highly abundant endophyte (OTU 1 and OTU 2 in Chapters $2 \& 3$, respectively), along with three other taxa, are previously undescribed and comprise a fam. nov. (Chapter 4). However, several new questions have emerged and the answers to these questions will further contribute to our understanding of this sparsely studied group of cryptic endosymbionts. 
Although competitive exclusion by OTU 1 may be one possible factor driving the results in the temporal study (Chapter 2), our in vitro competition assays do not corroborate this outcome: OTU 1 is outcompeted in the presence of every other endophyte tested. Still, we acknowledge that the intercellular (apoplast) space of the host plant is likely far different than in vitro conditions. Humidity is likely to be much higher in planta and temperature and light may fluctuate to a different degree affecting competitive outcomes within the host. A second potential explanation for the observed temporal turnover favoring OTU 1 may be changes in photosynthetic rates of the host. Polystichum likely undergoes the highest rates of photosynthesis in the early spring when juvenile tissue is emerging and rapidly expanding. Were this the case, levels of photosynthate would be higher and stomatal aperture would potentially be much greater at this stage allowing a higher number of endophyte colonists to arrive within the host. As photosynthetic rates slow in the summer and water conservation becomes more critical to the host, stomatal aperture would decrease thereby limiting the ability of later colonists to arrive. This "closing off" of host tissue may facilitate the spread of OTU 1 in warmer and drier summer conditions. Interestingly, the temporal turnover revealed in this study mimics succession in disturbed habitats for macroorganisms. The earliest colonists demonstrate community dynamics characteristic of ruderal species: they are superior at dispersal yet give way to more competitive, slow-growing climax species (Herms and Mattson 1992). Clearly more work is needed in the study of endophyte microsuccession patterns to determine if there is overlap with trends observed in macroecology. 
The Polystichum host may play a more active role in structuring its endosymbiotic community than previously assumed in other plant systems (Arnold and Lutzoni 2007). Specifically, the fern may be nutrient poor owing to its slow growth rates later in the season creating a depauperate community of endophytes. Further, the host may produce a high level of phenolics or tannins inhibiting microbial growth of most taxa save the most specialized endophytes. Lastly, some other novel antimicrobial compound may be produced either by the host or OTU 1 which deserves further exploration from an ecological and bioprospecting standpoint.

An examination of the spatial turnover of fungal endophytes across a $150 \mathrm{~km}$ gradient (Chapter 3) showed a surprisingly high degree of host specificity in the ferns when compared to the non-fern neighbors. These results further support the prediction that host filtering is occurring, leading to the persistence of OTU 2 (Catenospora polysticola). Clearly there are many endophytes comprising the regional species pool (2206 OTUs in this study), but only a specific subset of these are found colonizing the ferns. Future work should examine edaphic and climate variables related to patterns of community composition. Additionally, the phylogenetic distance of the host plant species could serve as a strong explanatory variable for the differences in community composition observed for the non-fern hosts. Lastly, the Polystichum host genetic structure of each population is unknown at this point which could also lead to differences in diversity and community composition in the fern hosts across the spatial gradient.

The Catenosporaceae fam. nov. falls within the Leotiomycetes, a large class of fungi within the largest fungal phylum: Ascomycota. Further, Catenosporaceae likely 
belongs to the Helotiales which is currently highly polyphyletic with a circumscription of the order desperately needed. Indeed, most of the closely-related taxa to Catenosporaceae belong to the Helotiales, yet many exceptions do exist (i.e. Leotiomycetes in. sed.). Since the family described in the present work possesses unique hyphae and conidia morphology when compared to the most closely-related taxa (e.g. Collophora, Gelatinomyces and Claussenomyces) including the terminal differentiation of hyphae into thalloconidia and unique colony characteristics including alternating dematiaceous and pink rings, and the presence of highly mucoid morphs in culture, we are confident that these taxa are both unique and undescribed. Additionally, these taxa are geographically isolated from the other most closely-related taxa and certainly phylogenetically divergent, leading to our conclusion that our monophyletic group is a new family and genus, comprising four species.

\section{Conclusions}

We agree with a recent call by Peay (2014) to begin coupling natural history/autecology experiments with NGS sequencing to avoid learning less and less about more and more data. The projects contained in the present work accurately exemplify this call. Not only were we able to demonstrate surprising broad scale endophyte community patterns through the culture-independent observational studies, but we were also able to further examine the performance of abundant taxa in culture conditions, examine their effects on the host plant and discover that the most abundant taxon in the fern host belongs to a new genus and family of fungi. Additionally, the strong temporal turnover and high degree of host specificity observed in Chapters $2 \& 3$ may be an example of co-evolution occurring 
between a host that strongly filters microbial colonists and an endophyte that is able to recolonize newly emerged host tissue by the timing of sporulation events in senescent material around the host plant. We have collected senescent material around the base of the host in the spring when initial colonization of OTU 1 occurs and isolations have revealed that C. polysticola and C. gilkeyae produce small black ascomata on this tissue. The pattern of existing as an endophyte within the host and continuing to persist after the tissue has senesced could be another example of the Foraging Ascomycete Hypothesis at work (Carroll 1999); however, in this instance, the endophyte is able to recolonize the same host repeatedly following sexual reproduction. Therefore, we suspect this may be a unique example of gyrofolius transmission; a term which we are first describing here. Additionally, if the Catenospora endophytes are able to delay leaf litter decomposition (LeRoy et al. 2011), this may further allow for the exclusion of neighboring understory plants competing for space against the Polystichum host. This would need to be confirmed with litter exclusion experiments to determine if different colonization patterns are seen in endophytes of litter-free Polystichum hosts. Since Polystichum is a long-lived plant, the ability of this tough host to exclude pathogens and favor Catenospora endophytes would be a remarkable adaptation. 


\section{References}

Agler, M. T., Ruhe, J., Kroll, S., Morhenn, C., Kim, S. T., Weigel, D., \& Kemen, E. M. (2016). Microbial Hub Taxa Link Host and Abiotic Factors to Plant Microbiome Variation. PLOS Biology, 14(1), e1002352.

Almario, J., Jeena, G., Wunder, J., Langen, G., Zuccaro, A., Coupland, G., \& Bucher, M. (2017). The root-associated fungal microbiota of non-mycorrhizal Arabis alpina and its contribution to plant phosphorus nutrition. Proceedings of the National Academy of Sciences of the United States of America.

Amend, A. S., Seifert, K. A., \& Bruns, T. D. (2010). Quantifying microbial communities with 454 pyrosequencing: Does read abundance count? Molecular Ecology, 19(24), $5555-5565$.

Anderson, M. J. (2001). A new method for non-parametric multivariate analysis of variance. Austral Ecology, 26(1), 32-46.

Anderson, M. J. (2006). Distance-based tests for homogeneity of multivariate dispersions. Biometrics, 62(1), 245-253.

Anderson, M. J., \& Walsh, D. C. I. (2017). PERMANOVA, ANOSIM , and the Mantel test in the face of heterogeneous dispersions : What null hypothesis are you testing? Ecological Monographs, 83(4), 557-574.

Arnold, A. E., \& Herre, E. A. (2003). Canopy cover and leaf age affect colonization by tropical fungal endophytes: Ecological pattern and process in Theobroma cacao (Malvaceae). Mycologia, 95(3), 388-398.

Arnold, A. E., Mejía, L. C., Kyllo, D., Rojas, E. I., Maynard, Z., Robbins, N., \& Herre, E. A. (2003). Fungal endophytes limit pathogen damage in a tropical tree. Proceedings of the National Academy of Sciences of the United States of America, 100(26), 15649-15654.

Arnold, A. E., Miadlikowska, J., Higgins, K. L., Sarvate, S. D., Gugger, P., Way, A., ... Lutzoni, F. (2009). A phylogenetic estimation of trophic transition networks for ascomycetous Fungi: Are lichens cradles of symbiotrophic Fungal diversification? Systematic Biology, 58(3), 283-297.

Arnold, A., \& Lutzoni, F. (2007). Diversity and host range of foliar fungal endophytes: are tropical leaves biodiversity hotspots? Ecology, 88(3), 541-549. 
Arnold, A., Maynard, Z., \& Gilbert, G. (2000). Are tropical fungal endophytes hyperdiverse? Ecology Letters 3, 267-274.

Aschehoug, E. T., Callaway, R. M., Newcombe, G., Tharayil, N., \& Chen, S. (2014). Fungal endophyte increases the allelopathic effects of an invasive forb. Oecologia, 175 (1), 285-291

Bálint, M., Bartha, L., O’Hara, R. B., Olson, M. S., Otte, J., Pfenninger, M., ... Schmitt, I. (2015). Relocation, high-latitude warming and host genetic identity shape the foliar fungal microbiome of poplars. Molecular Ecology, 24(1), 235-48.

Bálint, M., Schmidt, P. A., Sharma, R., Thines, M., \& Schmitt, I. (2014). An Illumina metabarcoding pipeline for fungi. Ecology and Evolution, 4(13), 2642-2653.

Bálint, M., Tiffin, P., Hallström, B., O’Hara, R. B., Olson, M. S., Fankhauser, J. D., ... Schmitt, I. (2013). Host Genotype Shapes the Foliar Fungal Microbiome of Balsam Poplar (Populus balsamifera). PLoS ONE, 8(1).

Bar-Massada, A., \& Belmaker, J. (2017). Non-stationarity in the co-occurrence patterns of species across environmental gradients. Journal of Ecology, 105(2), 391-399.

Baschien, C., Marvanová, L., \& Szewzyk, U. (2006). Phylogeny of selected aquatic hyphomycetes based on morphological and molecular data. Nova Hedwigia, 83(34), 311-352.

Baschien, C., Tsui, C. K. M., Gulis, V., Szewzyk, U., \& Marvanov??, L. (2013). The molecular phylogeny of aquatic hyphomycetes with affinity to the Leotiomycetes. Fungal Biology, 117(9), 660-672.

Bazzicalupo, A. L., Bálint, M., \& Schmitt, I. (2013). Comparison of ITS1 and ITS2 rDNA in 454 sequencing of hyperdiverse fungal communities. Fungal Ecology, 6(1), 102-109.

Becker, B., \& Marin, B. (2009). Streptophyte algae and the origin of embryophytes. Annals of Botany, 103(7), 999-1004.

Becker, M., Becker, Y., Green, K., \& Scott, B. (2016). The endophytic symbiont Epichloë festucae establishes an epiphyllous net on the surface of Lolium perenne leaves by development of an expressorium, an appressorium-like leaf exit structure. New Phytologist, 211(1) 240-254.

Benhamou, N. (2004). Potential of the Mycoparasite, Verticillium lecanii, to Protect Citrus Fruit Against Penicillium digitatum, the Causal Agent of Green Mold: A 
Comparison with the Effect of Chitosan. Phytopathology, 94(7), 693-705.

Benjamini, Y., \& Hochberg, Y. (1995). Controlling the False Discovery Rate: A Practical and Powerful Approach to Multiple Testing. Journal of the Royal Statistical Society, 57(1), 289-300.

Berg, G., Rybakova, D., Grube, M., \& Köberl, M. (2016). The plant microbiome explored: Implications for experimental botany. Journal of Experimental Botany, 67(4), 995-1002.

Bertness, M. D., \& Callaway, R. (1994). Positive interactions in communities. Trends in Ecology and Evolution, 9(5), 187-191.

Blackwell, M. (2011). The fungi: 1, 2, 3 ... 5.1 million species? American Journal of Botany, 98(3), 426-438.

Bordenstein, S. R., \& Theis, K. R. (2015). Host biology in light of the microbiome: Ten principles of holobionts and hologenomes. PLoS Biology, 13(8), 1-23.

Borer, E. T., Laine, A.-L., \& Seabloom, E. W. (2016). A multiscale approach to plant disease using the metacommunity concept. Annual Review of Phytopathology, 54(1), 397-418.

Brundrett, M. C. (2009). Mycorrhizal associations and other means of nutrition of vascular plants: Understanding the global diversity of host plants by resolving conflicting information and developing reliable means of diagnosis. Plant and Soil, 320(1), 37-77.

Bruns, T. D. (2001). ITS Reality. Inoculum, 52(6), 2-3.

Busby, P. E., Peay, K. G., \& Newcombe, G. (2015). Common foliar fungi of Poluplus trichocarpa modify Melampsora rust disease severity. New Phytologist, 209(4), $1681-1692$.

Busby, P. E., Ridout, M., \& Newcombe, G. (2015). Fungal endophytes: modifiers of plant disease. Plant Molecular Biology, 9(6), 645-655.

Busby, P. E., Soman, C., Wagner, M. R., Friesen, M. L., Kremer, J., Bennett, A., ... Dangl, J. L. (2017). Research priorities for harnessing plant microbiomes in sustainable agriculture, (5), 1-14.

Busby, P. E., Zimmerman, N., Weston, D. J., Jawdy, S. S., Houbraken, J., \& Newcombe, G. (2013). Leaf endophytes and Populus genotype affect severity of damage from the necrotrophic leaf pathogen, Drepanopeziza populi. Ecosphere, 4(10), art125. 
Callahan, B. J., McMurdie, P. J., \& Holmes, S. P. (2017). Exact sequence variants should replace operational taxonomic units in marker-gene data analysis. ISME Journal, 11(12), 2639-2643.

Camacho, C., Coulouris, G., Avagyan, V., Ma, N., Papadopoulos, J., Bealer, K., \& Madden, T. L. (2009). BLAST+: Architecture and applications. BMC Bioinformatics, 10, 1-9.

Capella-Gutiérrez, S., Silla-Martínez, J. M., \& Gabaldón, T. (2009). trimAl: A tool for automated alignment trimming in large-scale phylogenetic analyses. Bioinformatics, 25(15), 1972-1973.

Carroll, G. (1988). Fungal endophytes in stems and leaves: from latent pathogen to mutualistic symbiont. Ecology, 69(1), 2-9.

Carroll, G. C. (1999). The Foraging Ascomycete. In The 16th International Botanical Congress (p. Abstracts).

Castro, B. M., Moriuchi, K. S., Friesen, M. L., Badri, M., Nuzhdin, S. V., Strauss, S. Y., ... von Wettberg, E. (2013). Parental environments and interactions with conspecifics alter salinity tolerance of offspring in the annual medicago truncatula. Journal of Ecology, 101(5), 1281-1287.

Chase, J. M. (2003). Community assembly: When should history matter? Oecologia, $136(4), 489-498$.

Chauvet, E., Cornut, J., Sridhar, K. R., Selosse, M. A., \& B??rlocher, F. (2016). Beyond the water column: Aquatic hyphomycetes outside their preferred habitat. Fungal Ecology, 19, 112-127.

Che-castaldo, C., Crisafulli, C. M., Bishop, J. G., \& Fagan, W. F. (2015). What causes female bias in the secondary sex ratios of the dioecious woody shrub Salix sitchensis colonizing a primary successional landscape? American Journal of Botany, 102, 114.

Chesson, P. (2000). General theory of competitive coexistence in spatially-varying environments. Theoretical Population Biology, 58(3), 211-37.

Chesson, P., \& Huntly, N. (1997). The Roles of Harsh and Fluctuating Conditions in the Dynamics of Ecological Communities. The American Naturalist, 150(5), 519-553.

Chiarucci, A., Bacaro, G., Rocchini, D., \& Fattorini, L. (2008). Discovering and rediscovering the sample-based rarefaction formula in the ecological literature. 
Community Ecology, 9(1), 121-123.

Chiu, C.-H., \& Chao, A. (2016). Estimating and comparing microbial diversity in the presence of sequencing errors. PeerJ, 4, e1634.

Christian, N., Herre, E. A., Mejia, L. C., \& Clay, K. (2017). Exposure to the leaf litter microbiome of healthy adults protects seedlings from pathogen damage.

Proceedings of the Royal Society Biology, 284, 20170641.

Clay, K. (1988). Fungal Endophytes of Grasses: A Defensive Mutualism between Plants and Fungi. Ecology, 69(1), 10-16.

Clay, K. (1999). Fungal Endophyte Symbiosis and Plant Diversity in Successional Fields. Science, 285(5434), 1742-1744.

Cline, L. C., \& Zak, D. R. (2015). Initial colonization, community assembly and ecosystem function: fungal colonist traits and litter biochemistry mediate decay rate. Molecular Ecology, 24(19), 5045-5058.

Colwell, R. K., Chao, A., Gotelli, N. J., Lin, S. Y., Mao, C. X., Chazdon, R. L., \& Longino, J. T. (2012). Models and estimators linking individual-based and samplebased rarefaction, extrapolation and comparison of assemblages. Journal of Plant Ecology, 5(1), 3-21.

Cook, D., Beaulieu, W., Mott, I., Riet-Correa, F., Gardner, D., Grum, D., ... MarcolongoPereira, C. (2013). Production of the alkaloid swainsonine by a fungal endosymbiont of the Ascomycete order Chaetothyriales in the host Ipomoea carnea. Journal of Agricultural ..., 61, 3797-3803.

Cordier, T., Robin, C., Capdevielle, X., Desprez-Loustau, M. L., \& Vacher, C. (2012). Spatial variability of phyllosphere fungal assemblages: Genetic distance predominates over geographic distance in a European beech stand (Fagus sylvatica). Fungal Ecology, 5(5), 509-520.

Cosme, M., Lu, J., Erb, M., Stout, M. J., Franken, P., Wurst, S., \& Cosme, M. (2016). A fungal endophyte helps plants to tolerate root herbivory through changes in gibberellin and jasmonate signaling. New Phytologist.

Damm, U., Fourie, P. H., \& Crous, P. W. (2010). Coniochaeta (Lecythophora), collophora gen. nov. and phaeomoniella species associated with wood necroses of prunus trees. Persoonia: Molecular Phylogeny and Evolution of Fungi, 24, 60-80.

Davey, M. L., Heegaard, E., Halvorsen, R., Ohlson, M., \& Kauserud, H. (2012). Seasonal 
trends in the biomass and structure of bryophyte-associated fungal communities explored by 454 pyrosequencing. New Phytologist, 195, 844-856.

Del Olmo-Ruiz, M., \& Arnold, A. E. (2017). Community structure of fern-affiliated endophytes in three neotropical forests. Journal of Tropical Ecology, 33, 60-73.

Del Olmo-Ruiz, M., \& Arnold, a E. (2014). Interannual variation and host affiliations of endophytic fungi associated with ferns at La Selva, Costa Rica. Mycologia, 106(1), $8-21$.

Delaye, L., García-Guzmán, G., \& Heil, M. (2013). Endophytes versus biotrophic and necrotrophic pathogens-are fungal lifestyles evolutionarily stable traits? Fungal Diversity, 60(1), 125-135.

Dicosmo, F., Peredo, H., \& Minter, D. W. (1983). Cyclaneusma gen. nov., Naemacyclus and Lasiostictis, a nomenclatural problem resolved. European Journal of Forest Pathology, 13(4), 206-212.

Drummond, A. J., Suchard, M. A., Xie, D., \& Rambaut, A. (2012). Bayesian phylogenetics with BEAUti and the BEAST 1.7. Molecular Biology and Evolution, 29(8), 1969-1973.

Dunn, O. J. (1964). Multiple Comparisons Using Rank Sums. Technometrics, 6(3), 241252.

Dupont, P., Eaton, C. J., Wargent, J. J., Fechtner, S., Solomon, P., Schmid, J., ... Cox, M. P. (2015). Fungal endophyte infection of ryegrass reprograms host metabolism and alters development. New Phytologist, 208(4), 1227-12240

Edgar, R. (2016). SINTAX: a simple non-Bayesian taxonomy classifier for 16S and ITS sequences. bioRxiv, 74161.

Edgar, R. C. (2013). UPARSE: highly accurate OTU sequences from microbial amplicon reads. Nature Methods, 10(10), 996-998.

Edgar, R. C. (2016). UNOISE2: improved error-correction for Illumina 16S and ITS amplicon sequencing. bioRxiv, 81257 .

Edgar, R. C., \& Flyvbjerg, H. (2015). Error filtering, pair assembly and error correction for next-generation sequencing reads. Bioinformatics, 31(21), 3476-3482.

Ellis, A. M., Lounibos, L. P., \& Holyoak, M. (2006). Evaluating the long-term metacommunity dynamics of tree hole mosquitoes. Ecology, 87(10), 2582-2590.

Ellis, W. A. H., Melzer, A., \& Bercovitch, F. B. (2009). Spatiotemporal dynamics of 
habitat use by koalas: The checkerboard model. Behavioral Ecology and Sociobiology, 63(8), 1181-1188.

Farrar, K., Bryant, D., \& Cope-Selby, N. (2014). Understanding and engineering beneficial plant-microbe interactions: plant growth promotion in energy crops. Plant Biotechnology Journal, 12(9), 1193-206.

Fernandez, C. W., \& Kennedy, P. G. (2016). Revisiting the "Gadgil effect": Do interguild fungal interactions control carbon cycling in forest soils? New Phytologist, 209(4), 1382-1394.

Fernandez, H., Bertrand, A., \& Sanchez-Tames, R. (1993). In vitro regeneration of Asplenium nidus L. from gametophytic and sporophytic tissue. Scientia Horticulturae, 56, 71-77.

Ferrari, B. C., Zhang, C., \& van Dorst, J. (2011). Recovering greater fungal diversity from pristine and diesel fuel contaminated sub-antarctic soil through cultivation using both a high and a low nutrient media approach. Frontiers in Microbiology, 2(NOV), $1-14$.

Fesel, P. H., \& Zuccaro, A. (2016). Dissecting endophytic lifestyle along the parasitism/mutualism continuum in Arabidopsis. Current Opinion in Microbiology, $32,103-112$.

Fisher, P. (1996). Survival and spread of the endophyte Stagonospora pteridiicola in Pteridium aquilinum, other ferns and some flowering plants. New Phytologist, 132(1), 119-122.

Friesen, M. L. (2013). Microbially Mediated Plant Functional Traits. Molecular Microbial Ecology of the Rhizosphere, 1, 87-102.

Fukami, T. (2015). Historical contingency in community assembly : integrating niches, species pools, and priority effects. Annual Review of Ecology Evolution and Systematics, 46(April), 1-23.

Gernhard, T. (2008). The conditioned reconstructed process. Journal of Theoretical Biology, 253(4), 769-778.

Gilbert, S. F., Sapp, J., \& Tauber, A. I. (2012). A Symbiotic View of Life: We Have Never Been Individuals. The Quarterly Review of Biology, 87(4), 325-341.

Glassman, S. I., \& Martiny, J. B. H. (2018). Ecological patterns are robust to use of exact sequence variants versus operational taxonomic units. bioRxiv, 1-26. 
Glazebrook, J. (2005). Contrasting mechanisms of defense against biotrophic and necrotrophic pathogens. Annual Review of Phytopathology, 43, 205-27.

Gonczol, J., \& Revay, A. (1985). Gorgomyces, gen. nov., an Unusual Hyphomycete from Terrestrial Litter of Hungary. Nova Hedwigia, 41, 453-461.

González-Teuber, M., Jiménez-Alemán, G. H., \& Boland, W. (2014). Foliar endophytic fungi as potential protectors from pathogens in myrmecophytic Acacia plants. Communicative \& Integrative Biology, (November), 00-00.

Gotelli, N. J., \& Entsminger, G. L. (2003). Swap Algorithms in Null Model Analysis. Ecology, 84(2), 532-535.

Gräfenhan, T., Schroers, H. J., Nirenberg, H. I., \& Seifert, K. A. (2011). An overview of the taxonomy, phylogeny, and typification of nectriaceous fungi in Cosmospora, Acremonium, Fusarium, Stilbella, and Volutella. Studies in Mycology, 68, 79-113.

Gundel, P. E., Pérez, L. I., Helander, M., \& Saikkonen, K. (2013). Symbiotically modified organisms: nontoxic fungal endophytes in grasses. Trends in Plant Science, 18(8), 420-427.

Hanson, C. A., Fuhrman, J. A., Horner-Devine, M. C., \& Martiny, J. B. H. (2012). Beyond biogeographic patterns: processes shaping the microbial landscape. Nature Reviews Microbiology, 10(7), 497-506.

Harrison, M. J. (2005). Signaling in the arbuscular mycorrhizal symbiosis. Annual Review of Microbiology, 59, 19-42.

Hartley, S. E., \& Gange, A. C. (2009). Impacts of plant symbiotic fungi on insect herbivores: mutualism in a multitrophic context. Annual Review of Entomology, 54, $323-342$

Hassani, M. A., Durán, P., \& Hacquard, S. (2018). Microbial interactions within the plant holobiont. Microbiome, 6(58), 1-17.

Hawksworth, D. L., \& Rossman, A. Y. (1997). Where are all the undescribed fungi? Phytopathology, 87(9), 888-91.

Hawksworth, D. L., \& Sherwood, M. A. (1981). A reassessment of three widespread resinicolous discomycetes. Canadian Journal of Botany, 59(3), 357-372.

Herms, D., \& Mattson, W. (1992). The dilemma of plants: to grow or defend. Quarterly Review of Biology, 67(3), 283-335.

Hibbett, D. S., Binder, M., Bischoff, J. F., Blackwell, M., Cannon, P. F., Eriksson, O. E., 112 
... Zhang, N. (2007). A higher-level phylogenetic classification of the Fungi. Mycological Research, 111(5), 509-547.

Higgins, K. L., Arnold, a E., Miadlikowska, J., Sarvate, S. D., \& Lutzoni, F. (2007). Phylogenetic relationships, host affinity, and geographic structure of boreal and arctic endophytes from three major plant lineages. Molecular Phylogenetics and Evolution, 42(2), 543-55.

Hill, M. O. (1973). Diversity and Evenness : A Unifying Notation and Its Consequences. Ecology, 54(2), 427-432.

Hosaka, K., Bates, S. T., Beever, R. E., Castellano, M. A., Colgan, W., Dominguez, L. S., ... Trappe, J. M. (2006). Molecular phylogenetics of the gomphoid-phalloid fungi with an establishment of the new subclass Phallomycetidae and two new orders. Mycologia, 98(6), 949-959.

Hubbell, S. P. (2001). The Unified Neutral Theory of Biodiversity and Biogeography. Princeton, NJ: Princeton University Press.

Johnston, P. R. (2006). Rhytismatales of Australia: The genus Marthamyces. Australian Systematic Botany, 19(2), 135-146.

Johnston, P. R., Seifert, K. a, Stone, J. K., Rossman, A. Y., \& Marvanová, L. (2014). Recommendations on generic names competing for use in Leotiomycetes (Ascomycota). IMA Fungus, 5(1), 91-120.

Jooste, W. J., \& Merwe, W. J. J. Van Der. (1990). Ultrastructure of the conidiogenesis and conidia of Anguillospora pseudolongissima and Flagellospora penicillioides. South African Journal of Botany, 56(3), 319-325.

Jumpponen, A., \& Jones, K. L. (2010). Seasonally dynamic fungal communities in the Quercus macrocarpa phyllosphere differ between urban and nonurban environments. The New Phytologist, 186(2), 496-513.

Kearse, M., Moir, R., Wilson, A., Stones-Havas, S., Cheung, M., Sturrock, S., ... Drummond, A. (2012). Geneious Basic: An integrated and extendable desktop software platform for the organization and analysis of sequence data. Bioinformatics, 28(12), 1647-1649.

Kennedy, P. (2010). Ectomycorrhizal fungi and interspecific competition: Species interactions, community structure, coexistence mechanisms, and future research directions. New Phytologist, 187(4), 895-910. 
Kennedy, P. G., Nguyen, N. H., Cohen, H., Smith, D. P., \& Peay, K. G. (2014). Missing checkerboards: an absence of competitive signal in stress-associated ectomycorrhizal fungal communities. ISME Journal, 1-21.

Khan, Z., Rho, H., Firrincieli, A., Hung, S. H., Luna, V., Masciarelli, O., ... Doty, S. L. (2016). Growth enhancement and drought tolerance of hybrid poplar upon inoculation with endophyte consortia. Current Plant Biology, (August).

Kiers, E., \& Heijden, M. (2006). Mutualistic Stability In The Arbuscular Mycorrhizal Symbiosis: Exploring Hypotheses Of Evolutionary Cooperation. Ecology, 87(7), $1627-1636$.

Kivlin, S. N., Winston, G. C., Goulden, M. L., \& Treseder, K. K. (2014). Environmental filtering affects soil fungal community composition more than dispersal limitation at regional scales. Fungal Ecology, 12(C), 14-25.

Klekowski, E. J. (1969). Reproductive Biology of the Pteridophyta. III. A study of the Blechnaceae. Botanical Journal of the Linnean Society, 62, 361-377.

Kneitel, J. M., \& Miller, T. E. (2003). Dispersal Rates Affect Species Composition in Metacommunities of Sarracenia purpurea Inquilines. The American Naturalist, 162(2), 165-171.

Knoth, J. L., Kim, S. H., Ettl, G. J., \& Doty, S. L. (2014). Biological nitrogen fixation and biomass accumulation within poplar clones as a result of inoculations with diazotrophic endophyte consortia. New Phytologist.

Kohout, P., TěŠitelová, T., Roy, M., Vohník, M., \& Jersáková, J. (2013). A diverse fungal community associated with Pseudorchis albida (Orchidaceae) roots. Fungal Ecology, 6(1), 50-64.

Koide, R. T., Ricks, K. D., \& Davis, E. R. (2017). Climate and dispersal in fl uence the structure of leaf fungal endophyte communities of Quercus gambelii in the eastern Great Basin, USA. Fungal Ecology, 30, 19-28.

Kõljalg, U., Nilsson, R. H., Abarenkov, K., Tedersoo, L., Taylor, A. F. S., Bahram, M., ... Larsson, K. H. (2013). Towards a unified paradigm for sequence-based identification of fungi. Molecular Ecology, 22(21), 5271-5277.

Koskella, B., Hall, L. J., \& Metcalf, C. J. E. (2017). The microbiome beyond the horizon of ecological and evolutionary theory. Nature Ecology and Evolution, 1(11), 16061615. 
Krings, M., Taylor, T. N., Hass, H., Kerp, H., Dotzler, N., \& Hermsen, E. J. (2007). Fungal endophytes in a 400-million-yr-old land plant: infection pathways, spatial distribution, and host responses. The New Phytologist, 174(3), 648-57.

Kruskal, W. H., \& Wallis, W. A. (1952). Use of Ranks in One-Criterion Variance Analysis. Journal of the American Statistical Association, 47(260), 583-621.

Kučera, V., Lizoň, P., Tomšovský, M., Kučera, J., \& Gaisler, J. (2014). Re-evaluation of the morphological variability of Microglossum viride and M. griseoviride sp. nov. Mycologia, 106(2), 282-290.

Lanfear, R., Calcott, B., Ho, S. Y. W., \& Guindon, S. (2012). PartitionFinder: Combined selection of partitioning schemes and substitution models for phylogenetic analyses. Molecular Biology and Evolution, 29(6), 1695-1701.

Lantz, H., Johnston, P. R., Park, D., \& Minter, D. W. (2011). Molecular phylogeny reveals a core clade of Rhytismatales. Mycologia, 103(1), 57-74.

Leibold, M. A., Holyoak, M., Mouquet, N., Amarasekare, P., Chase, J. M., Hoopes, M. F., ... Gonzalez, A. (2004). The metacommunity concept: A framework for multiscale community ecology. Ecology Letters, 7(7), 601-613.

LeRoy, C. J., Fischer, D. G., Halstead, K., Pryor, M., Bailey, J. K., \& Schweitzer, J. A. (2011). A fungal endophyte slows litter decomposition in streams. Freshwater Biology, 56(7), 1426-1433.

Limm, E. B., \& Dawson, T. E. (2010). Polystichum munitum (Dryopteridaceae) varies geographically in its capacity to absorb fog water by foliar uptake within the redwood forest ecosystem. American Journal of Botany, 97(7), 1121-8.

Little, D. P., \& Barrington, D. S. (2003). Major evolutionary events in the origin and diversification of the fern genus Polystichum (Dryopteridaceae). American Journal of Botany, 90(3), 508-514.

Lorch, J. M., Meteyer, C. U., Behr, M. J., Boyles, J. G., Cryan, P. M., Hicks, A. C., ... Blehert, D. S. (2011). Experimental infection of bats with Geomyces destructans causes white-nose syndrome. Nature, 480(7377), 376-378.

MacArthur, R. H., \& Wilson, E. O. (1967). The Theory of Island Biogeography. Princeton University Press.

Martin, M. (2011). Cutadapt removes adapter sequences from high-throughput sequencing reads. EMBnet.journal, 17(1), 10. 
Martiny, J. B. H., Bohannan, B. J. M., Brown, J. H., Colwell, R. K., Fuhrman, J. a, Green, J. L., ... Staley, J. T. (2006). Microbial biogeography: putting microorganisms on the map. Nature Reviews. Microbiology, 4(February), 102-112.

Marvanová, L., \& Descals, E. (1985). New and critical taxa of aquatic hyphomycetes. Botanical Journal of the Linnean Society, 91(1-2), 1-23.

Matheny, B. P., Liu, Y. J., Ammirati, J. F., \& Hall, B. D. (2002). Using RPB1 sequences to improve phylogenetic inference among mushrooms (Inocybe, Agaricales). American Journal of Botany, 89(4), 688-698.

May, G. (2016). Here come the commensals. American Journal of Botany, 103(10), 1709-1711.

McMullin, D. R., Nguyen, H. D. T., Daly, G. J., Menard, B. S., \& Miller, J. D. (2018). Detection of foliar endophytes and their metabolites in Picea and Pinus seedling needles. Fungal Ecology, 31, 1-8.

McMurdie, P. J., \& Holmes, S. (2014). Waste not, want not: why rarefying microbiome data is inadmissible. PLoS Computational Biology, 10(4), e1003531.

Mehltreter, K., Walker, L. R., \& Sharpe, J. M. (2010). Fern Ecology.

Meiser, A., Bálint, M., \& Schmitt, I. (2014). Meta-analysis of deep-sequenced fungal communities indicates limited taxon sharing between studies and the presence of biogeographic patterns. New Phytologist, 201(2), 623-635.

Mejía, L. C., Rojas, E. I., Maynard, Z., Bael, S. Van, Arnold, a. E., Hebbar, P., ... Herre, E. A. (2008). Endophytic fungi as biocontrol agents of Theobroma cacao pathogens. Biological Control, 46(1), 4-14.

Migliaro, G., \& Gabriel Y Galán, J. M. (2012). Gametophyte development and reproduction of the Asian fern Polystichum polyblepharum (Roem. ex Kunze) C. Presl (Dryopteridaceae, Polypodiopsida). Plant Biosystems, 146(2), 368-373.

Mihaljevic, J. R. (2012). Linking metacommunity theory and symbiont evolutionary ecology. Trends in Ecology and Evolution, 27(6), 323-329.

Minter, D. W. (2003). Propolis and Marthamyces gen. nov. (Rhytismatales). Mycotaxon, $87,43-52$.

Moeller, H. V., \& Peay, K. G. (2016). Competition-function tradeoffs in ectomycorrhizal fungi. PeerJ, 4, e2270.

Moon, D. C., Barnouti, J., \& Younginger, B. (2013). Context-dependent effects of 
mycorrhizae on herbivore density and parasitism in a tritrophic coastal study system. Ecological Entomology, 38(1), 31-39.

Moran, N. A., \& Sloan, D. B. (2015). The Hologenome Concept: Helpful or Hollow? PLoS Biology, 13(12), 1-10.

Mouquet, N., \& Loreau, M. (2002). Coexistence in Metacommunities: The Regional Similarity Hypothesis. The American Naturalist, 159(4), 420-426.

Mouquet, N., \& Loreau, M. (2003). Community Patterns in Source-Sink Metacommunities. The American Naturalist, 162(5), 544-557.

Mucciarelli, M., Scannerini, S., Bertea, C., \& Maffei, M. (2003). In vitro and in vivo peppermint (Mentha piperita) growth promotion by nonmycorrhizal fungal colonization. New Phytologist, 158(3), 579-591.

Navarro-Meléndez, A. L., \& Heil, M. (2014). Symptomless Endophytic Fungi Suppress Endogenous Levels of Salicylic Acid and Interact With the Jasmonate-Dependent Indirect Defense Traits of Their Host, Lima Bean (Phaseolus lunatus). Journal of Chemical Ecology, 40(7), 816-825.

Nguyen, N., Smith, D., Peay, K., \& Kennedy, P. (2014). Parsing ecological signal from noise in next generation amplicon sequencing. New Phytologist.

Nilsson, R. H., Hyde, K. D., Pawłowska, J., Ryberg, M., Tedersoo, L., Aas, A. B., ... Abarenkov, K. (2014). Improving ITS sequence data for identification of plant pathogenic fungi. Fungal Diversity, 67(1), 11-19.

Nilsson, R. H., Kristiansson, E., Ryberg, M., Hallenberg, N., \& Larsson, K. H. (2008). Intraspecific ITS variability in the Kingdom Fungi as expressed in the international sequence databases and its implications for molecular species identification.

Evolutionary Bioinformatics, 2008(4), 193-201.

O’Brien, H. E., Parrent, J. L., Jackson, J. A., Moncalvo, J., \& Vilgalys, R. (2005). Fungal Community Analysis by Large-Scale Sequencing of Environmental Samples. Applied and Environmental Microbiology, 71(9), 5544-5550.

O’Donnell, K., Sarver, B. A. J., Brandt, M., Chang, D. C., Noble-Wang, J., Park, B. J., ... Ward, T. J. (2007). Phylogenetic diversity and microsphere array-based genotyping of human pathogenic fusaria, including isolates from the multistate contact lensassociated U.S. keratitis outbreaks of 2005 and 2006. Journal of Clinical Microbiology, 45(7), 2235-2248. 
Oksanen, J. F. G., Blanchet, R., Kindt, P., Legendre, R. B., O’Hara, G. L., Simpson, P., ... Wagner, H. (2011). Vegan: Community Ecology Package. R package version 2.3-0.

Oono, R., Lutzoni, F., Arnold, A. E., Kaye, L., U’Ren, J. M., May, G., \& Carbone, I. (2014). Genetic variation in horizontally transmitted fungal endophytes of pine needles reveals population structure in cryptic species. American Journal of Botany, 101(8), 1362-1374.

Oulhen, N., Schulz, B. J., \& Carrier, T. J. (2016). English translation of Heinrich Anton de Bary’s 1878 speech, "Die Erscheinung der Symbiose” ("De la symbiose”). Symbiosis, 69(3), 131-139.

Ownley, B. H., Gwinn, K. D., \& Vega, F. E. (2009). Endophytic fungal entomopathogens with activity against plant pathogens: ecology and evolution. BioControl, 55(1), $113-128$.

Paden, J. W., \& Tylutki, E. E. (1969). Idaho Discomycetes. II. Mycologia, 61(4), 683693.

Pan, J. J., \& May, G. (2009). Fungal-fungal associations affect the assembly of endophyte communities in maize (Zea mays). Microbial Ecology, 58(3), 668-678.

Panaccione, D. G., Johnson, R. D., Wang, J., Young, C. a, Damrongkool, P., Scott, B., \& Schardl, C. L. (2001). Elimination of ergovaline from a grass-Neotyphodium endophyte symbiosis by genetic modification of the endophyte. Proceedings of the National Academy of Sciences of the United States of America, 98(22), 1282012825.

Paparu, P., Dubois, T., Coyne, D., \& Viljoen, A. (2007). Defense-related gene expression in susceptible and tolerant bananas (Musa spp.) following inoculation with nonpathogenic Fusarium oxysporum endophytes and challenge with Radopholus similis. Physiological and Molecular Plant Pathology, 71(2007), 149-157.

Peay, K. G. (2014). Back to the future: Natural history and the way forward in modern fungal ecology. Fungal Ecology, 12(C), 4-9.

Peay, K. G., \& Bruns, T. D. (2014). Spore dispersal of basidiomycete fungi at the landscape scale is driven by stochastic and deterministic processes and generates variability in plant-fungal interactions. New Phytologist, 204(1), 180-191.

Peay, K. G., Bruns, T. D., Kennedy, P. G., Bergemann, S. E., \& Garbelotto, M. (2007). A strong species-area relationship for eukaryotic soil microbes: Island size matters for 
ectomycorrhizal fungi. Ecology Letters, 10(6), 470-480.

Peay, K. G., Garbelotto, M., \& Bruns, T. D. (2010). Evidence of dispersal limitation in soil microorganisms: Isolation reduces species richness on mycorrhizal tree islands. Ecology, 91(12), 3631-3640.

Peay, K. G., Kennedy, P. G., \& Talbot, J. M. (2016). Dimensions of biodiversity in the Earth mycobiome. Nature Reviews Microbiology, 14(7), 434-447.

Pedruski, M. T., \& Arnott, S. E. (2011). The effects of habitat connectivity and regional heterogeneity on artificial pond metacommunities. Oecologia, 166(1), 221-228.

Peñuelas, J., Rico, L., Ogaya, R., Jump, A. S., \& Terradas, J. (2012). Summer season and long-term drought increase the richness of bacteria and fungi in the foliar phyllosphere of Quercus ilex in a mixed Mediterranean forest. Plant Biology, 14(4), 565-575.

Peršoh, D. (2013). Factors shaping community structure of endophytic fungi-evidence from the Pinus-Viscum-system. Fungal Diversity, 60(1), 55-69.

Peršoh, D. (2015). Plant-associated fungal communities in the light of meta'omics. Fungal Diversity, 75(1), 1-25.

Petersen, R. H. (1963). Aquatic Hyphomycetes from North America: III . Phialosporae and Miscellaneous Species Mycologia, 55(5), 570-581.

Pirozynski, K., \& Malloch, D. (1975). The origin of land plants: a matter of mycotrophism. Biosystems, 6, 153-164.

Qadri, M., Rajput, R., Abdin, M. Z., Vishwakarma, R. a, \& Riyaz-Ul-Hassan, S. (2014). Diversity, Molecular Phylogeny, and Bioactive Potential of Fungal Endophytes Associated with the Himalayan Blue Pine (Pinus wallichiana). Microbial Ecology.

Queloz, V., Sieber, T. N., Holdenrieder, O., McDonald, B. A., \& Grünig, C. R. (2011). No biogeographical pattern for a root-associated fungal species complex. Global Ecology and Biogeography, 20(1), 160-169.

Redman, R. S., Kim, Y. O., Woodward, C. J., Greer, C., Espino, L., Doty, S. L., \& Rodriguez, R. J. (2011). Increased fitness of rice plants to abiotic stress via habitat adapted symbiosis: a strategy for mitigating impacts of climate change. PloS One, 6(7), e14823.

Redman, R. S., Sheehan, K. B., Stout, R. G., Rodriguez, R. J., \& Henson, J. M. (2002). Thermotolerance generated by plant/fungal symbiosis. Science (New York, N.Y.), 
$298(5598), 1581$.

Rodriguez, R. J., Henson, J., Van Volkenburgh, E., Hoy, M., Wright, L., Beckwith, F., ... Redman, R. S. (2008). Stress tolerance in plants via habitat-adapted symbiosis. The ISME Journal, 2(4), 404-16.

Rodriguez, R. J., White, J. F., Arnold, A., \& Redman, R. S. (2009). Fungal endophytes: diversity and functional roles. The New Phytologist, 182(2), 314-30.

Rodriguez, R., Redman, R., \& Henson, J. (2004). The role of fungal symbioses in the adaptation of plants to high stress environments. ... Adaptation Strategies for Global ..., 9, 261-272.

Roldan, A. (1989). A New Addition to the Genus Gorgomyces. Mycotaxon, 34(2), 381385.

Rueden, C. T., Schindelin, J., Hiner, M. C., DeZonia, B. E., Walter, A. E., Arena, E. T., \& Eliceiri, K. W. (2017). ImageJ2: ImageJ for the next generation of scientific image data. BMC Bioinformatics, 18(1), 1-26.

Sanoamuang, N., Jitjak, W., Rodtong, S., \& Whalley, A. J. S. (2013). Gelatinomyces siamensis gen. sp. nov. (Ascomycota, Leotiomycetes, incertae sedis) on bamboo in Thailand. IMA Fungus, 4(1), 71-87.

Sarhan, M. S., Mourad, E. F., Hamza, M. A., Youssef, H. H., Scherwinski, A. C., ElTahhan, M., ... Hegazi, N. A. (2016). Plant powder teabags: A novel and practical approach to resolve culturability and diversity of rhizobacteria. Physiologia Plantarum.

Sati, S. C., \& Belwal, M. (2005). Aquatic hyphomycetes as endophytes of riparian plant roots. Mycologia, 97(1), 45-49.

Schindelin, J., Arganda-Carreras, I., Frise, E., Kaynig, V., Longair, M., Pietzsch, T., ... Cardona, A. (2012). Fiji: an open-source platform for biological-image analysis. Nature Methods, 9(7), 676-682.

Schirrmann, M. K., Zoller, S., Fior, S., \& Leuchtmann, A. (2014). Genetic Evidence for Reproductive Isolation Among Sympatric Epichloë Endophytes as Inferred from Newly Developed Microsatellite Markers. Microbial Ecology.

Schlegel, M., Dubach, V., von Buol, L., \& Sieber, T. N. (2016). Effects of endophytic fungi on the ash dieback pathogen. FEMS Microbiology Ecology, 92(9), 1-18.

Schmidt, P. A., Bálint, M., Greshake, B., Bandow, C., Römbke, J., \& Schmitt, I. (2013). 
Illumina metabarcoding of a soil fungal community. Soil Biology and Biochemistry, $65,128-132$.

Schuettpelz, E., \& Pryer, K. M. (2009). Evidence for a Cenozoic radiation of ferns in an angiosperm-dominated canopy. Proceedings of the National Academy of Sciences of the United States of America, 106(27), 11200-11205.

Schwelm, A., Barron, N. J., Baker, J., Dick, M., Long, P. G., Zhang, S., \& Bradshaw, R. E. (2009). Dothistromin toxin is not required for dothistroma needle blight in Pinus radiata. Plant Pathology, 58(2), 293-304.

Seena, S., \& Monroy, S. (2016). Preliminary insights into the evolutionary relationships of aquatic hyphomycetes and endophytic fungi. Fungal Ecology, 19, 128-134.

Selosse, M. a, \& Le Tacon, F. (1998). The land flora: a phototroph-fungus partnership? Trends in Ecology \& Evolution, 13(1), 15-20.

Sessa, E. B., Testo, W. L., \& Jr, J. E. W. (2016). On the widespread capacity for, and functional significance of, extreme inbreeding in ferns.

Sharma, G., \& Pandey, R. R. (2010). Influence of culture media on growth, colony character and sporulation of fungi isolated from decaying vegetable wastes. Journal of Yeast and Fungal Research, 1, 157-164.

Shokralla, S., Porter, T. M., Gibson, J. F., Dobosz, R., Janzen, D. H., Hallwachs, W., ... Hajibabaei, M. (2015). Massively parallel multiplex DNA sequencing for specimen identification using an Illumina MiSeq platform. Scientific Reports, 5.

Smith, D. P., \& Peay, K. G. (2014). Sequence Depth, Not PCR Replication, Improves Ecological Inference from Next Generation DNA Sequencing. PLoS ONE, 9(2), e90234.

Smith, G. R., Steidinger, B. S., Bruns, T. D., \& Peay, K. G. (2018). Competitioncolonization tradeoffs structure fungal diversity. The ISME Journal.

Smith, S., Smith, F., \& Jakobsen, I. (2003). Mycorrhizal Fungi Can Dominate Phosphate Supply to Plants Irrespective of Growth Responses. Plant Physiology, 133(1), 1620.

Sneck, M. E., Rudgers, J. A., Young, C. A., \& Miller, T. E. X. (2017). Variation in the Prevalence and Transmission of Heritable Symbionts Across Host Populations in Heterogeneous Environments. Microbial Ecology.

Sokolski, S., Piché, Y., Chauvet, É., \& Bérubé, J. A. (2006). A fungal endophyte of black 
spruce (Picea mariana) needles is also an aquatic hyphomycete. Molecular Ecology, 15(7), 1955-1962.

Soltis, P. S., \& Soltis, D. E. (1987). Population Structure and Estimates of Gene Flow in the Homosporous Fern Polystichum munitum. Evolution, 41(3), 620-629.

Song, Z., Kennedy, P. G., Liew, F. J., \& Schilling, J. S. (2017). Fungal endophytes as priority colonizers initiating wood decomposition. Functional Ecology, 31(2), 407418.

Song, Z., Schlatter, D., Kennedy, P., Kinkel, L. L., Kistler, H. C., Nguyen, N., \& Bates, S. T. (2015). Effort versus reward Preparing samples for fungal community characterization in high-throughput sequencing surveys of soils. PLoS ONE, 10(5), $1-13$.

Stamatakis, A. (2014). RAxML version 8: A tool for phylogenetic analysis and postanalysis of large phylogenies. Bioinformatics, 30(9), 1312-1313.

Stegen, J. C., Freestone, A. L., Crist, T. O., Anderson, M. J., Chase, J. M., Comita, L. S., ... Vellend, M. (2013). Stochastic and deterministic drivers of spatial and temporal turnover in breeding bird communities. Global Ecology and Biogeography, 22(2), 202-212.

Stone, L., \& Roberts, A. (1990). The Checkerboard Score and Species Distributions. Oecologia, 85(1), 74-79.

Strobel, G., \& Daisy, B. (2003). Bioprospecting for microbial endophytes and their natural products. Microbiology and Molecular Biology Reviews, 67(4).

Subramanian, C. V. (1983). Hyphomycetes, Taxonomy and Biology.

Suija, A., Ertz, D., Lawrey, J. D., \& Diederich, P. (2014). Multiple origin of the lichenicolous life habit in Helotiales, based on nuclear ribosomal sequences. Fungal Diversity, 70(1), 55-72.

Sullivan, T. J., \& Faeth, S. H. (2004). Gene flow in the endophyte Neotyphodium and implications for coevolution with Festuca arizonica. Molecular Ecology, 13(3), 649656.

Sun, X., \& Guo, L.-D. (2012). Endophytic fungal diversity: review of traditional and molecular techniques. Mycology, 3(1), 65-76.

Suryanarayanan, T., \& Thennarasan, S. (2004). Temporal variation in endophyte assemblages of Plumeria rubra leaves. Fungal Diversity, 15, 197-204. 
Swatzell, L. J., Powell, M. J., \& Kiss, J. Z. (1996). The relationship of endophytic fungi to the gametophyte of the fern Schizaea pusilla. International Journal of Plant Sciences, 157(1), 53-62.

Terhonen, E., Sipari, N., \& Asiegbu, F. O. (2016). Inhibition of phytopathogens by fungal root endophytes of Norway spruce. Biological Control, 99, 53-63.

Thomas, D. C., Vandegrift, R., Ludden, A., Carroll, G. C., \& Roy, B. A. (2016). Spatial Ecology of the Fungal Genus Xylaria in a Tropical Cloud Forest. Biotropica, $0(0)$, $1-13$.

Thompson, L. R., Sanders, J. G., McDonald, D., Amir, A., Ladau, J., Locey, K. J., ... Zhao, H. (2017). A communal catalogue reveals Earth's multiscale microbial diversity. Nature.

Tilman, D. (2007). Resource competition and plant traits: A response to Craine et al. 2005. Journal of Ecology, 95(2), 231-234.

U'Ren, J. M., \& Arnold, A. E. (2016). Diversity, taxonomic composition, and functional aspects of fungal communities in living, senesced, and fallen leaves at five sites across North America. PeerJ, 4, e2768.

Urban, M. C., Leibold, M. A., Amarasekare, P., De Meester, L., Gomulkiewicz, R., Hochberg, M. E., ... Wade, M. J. (2008). The evolutionary ecology of metacommunities. Trends in Ecology and Evolution, 23(6), 311-317.

Van der Gucht, K., Cottenie, K., Muylaert, K., Vloemans, N., Cousin, S., Declerck, S., ... De Meester, L. (2007). The power of species sorting: local factors drive bacterial community composition over a wide range of spatial scales. Proceedings of the National Academy of Sciences of the United States of America, 104(51), 2040420409.

Vandenkoornhuyse, P., Quaiser, A., Duhamel, M., Le Van, A., \& Dufresne, A. (2015). The importance of the microbiome of the plant holobiont. New Phytologist, 206(4), 1196-1206.

Vanschoenwinkel, B., De Vries, C., Seaman, M., \& Brendonck, L. (2007). The role of metacommunity processes in shaping invertebrate rock pool communities along a dispersal gradient. Oikos, 116(8), 1255-1266.

Vellinga, E., Kuyper, T. W., Vellinga, E. C., Kuyper, T. W., Ammirati, J., Desjardin, D. E., ... Lebel, T. (2015). Six simple guidelines for introducing new genera of fungi Six simple guidelines for introducing new genera of fungi. IMA Fungus, 6(2), 65- 
68.

Veloso, J., \& Díaz, J. (2012). Fusarium oxysporum Fo47 confers protection to pepper plants against Verticillium dahliae and Phytophthora capsici, and induces the expression of defence genes. Plant Pathology, 61(July 2011), 281-288.

Verkley, G. J. M. (1994). Ultrastructure of the ascus apical apparatus in Leotia lubrica and some Geoglossaceae (Leotiales, Ascomycotina). Persoonia, 15(4), 405-430.

Vidal, S., \& Jaber, L. R. (2015). Entomopathogenic fungi as endophytes: plantendophyte-herbivore interactions and prospects for use in biological control. Current Science, 109(1), 46-54.

Vincent, J. B., Weiblen, G. D., \& May, G. (2015). Host associations and beta diversity of fungal endophyte communities in New Guinea rainforest trees. Molecular Ecology, 25(3).

Wang, Z., Binder, M., Schoch, C. L., Johnston, P. R., Spatafora, J. W., \& Hibbett, D. S. (2006). Evolution of helotialean fungi (Leotiomycetes, Pezizomycotina): A nuclear rDNA phylogeny. Molecular Phylogenetics and Evolution, 41(2), 295-312.

Wang, Z., Johnston, P. R., Takamatsu, S., Spatafora, J. W., \& Hibbett, D. S. (2006). Toward a phylogenetic classification of the leotiomycetes based on rDNA data. Mycologia, 98(6), 1065-1075.

Wardle, D. A., \& Parkinson, D. (1992). The influence of the herbicide glyphosate on interspecific interactions between four soil fungal species. Mycological Research, 96, 180-186.

Warner, R. R., \& Chesson, P. L. (1985). Coexistence Mediated by Recruitment Fluctuations : A Field Guide to the Storage Effect. The American Naturalist, 125(6), 769-787.

Watkins, J. E., Mack, M. C., Sinclair, T. R., \& Mulkey, S. S. (2007). Ecological and evolutionary consequences of desiccation tolerance in tropical fern gametophytes. New Phytologist, 176(3), 708-717.

Wells, K., \& Oberwinkler, F. (1982). Tremelloscypha gelatinosa, a species of a new family Sebacinaceae. Mycologia, 74(2), 325-331.

White, T. J., Bruns, T., Lee, S., \& Taylor, J. W. (1990). Amplification and direct sequencing of fungal ribosomal RNA genes for phylogenetics. In PCR Protocols: A Guide to Methods and Applications (pp. 315-322). 
Wickham, H. (2009). ggplot2: Elegant Graphics for Data Analysis. New York: SpringerVerlag.

Wilson, D. (1995). Endophyte: The Evolution of a Term, and Clarification of Its Use and Definition. Oikos, 73, 274-276.

Winfred, R., Taylor, T. N., Hass, H., \& Kerp, H. (1994). Four hundred-million-year-old vesicular abuscular mycorrhizae. Proceedings of the National Academy of Science, 91(December), 11841-11843.

Wright, D. H., Patterson, B. D., Mikkelson, G. M., Cutler, A., \& Atmar, W. (1998). A comparative analysis of nested subset patterns of species composition. Oecologia, $113(1), 1-20$.

Younginger, B., Barnouti, J., \& Moon, D. C. (2009). Interactive effects of mycorrhizal fungi, salt stress, and competition on the herbivores of Baccharis halimifolia. Ecological Entomology, 34(5), 580-587.

Younginger, B. S., \& Ballhorn, D. J. (2017). Fungal endophyte communities in the temperate fern Polystichum munitum show early colonization and extensive temporal turnover. American Journal of Botany, 104(8), 1188-1194.

Zapalski, M. K. (2011). Is absence of proof a proof of absence? Comments on commensalism. Palaeogeography, Palaeoclimatology, Palaeoecology, 302(3-4), $484-488$.

Zhang, W., Wang, H.-W., Wang, X.-X., Xie, X.-G., Ashaduzzaman, S. M., Xu, R.-S., \& Dai, C.-C. (2015). Enhanced nodulation of peanut when co-inoculated with fungal endophyte Phomopsis liquidambari and bradyrhizobium. Plant Physiology and Biochemistry, 98, 1-11.

Zhou, W.-N., White, J. F., Soares, M. A., Torres, M. S., Zhou, Z.-P., \& Li, H.-Y. (2015). Diversity of fungi associated with plants growing in geothermal ecosystems and evaluation of their capacities to enhance thermotolerance of host plants. Journal of Plant Interactions, 10(1), 305-314.

Zimmerman, N. B., \& Vitousek, P. M. (2012). Fungal endophyte communities reflect environmental structuring across a Hawaiian landscape. Proceedings of the National Academy of Sciences of the United States of America, 109(32), 13022-7.

Zubek, S., Piątek, K., Naks, P., Heise, W., Wayda, M., \& Mleczko, P. (2010). Fungal Root Endophyte Colonization of Fern and Lycophyte Species from the Celaque National Park in Honduras. American Fern Journal, 100(2), 126-136. 
Appendix A. Neighboring plant taxonomy from Chapter 3. Each number corresponds a neighboring plant to each P. munitum host sampled.

\begin{tabular}{|c|c|c|c|c|c|c|c|c|}
\hline & \multicolumn{2}{|r|}{ Coast } & \multicolumn{2}{|r|}{ Coast Range } & \multicolumn{2}{|r|}{ MSH control } & \multicolumn{2}{|r|}{ MSH impacted } \\
\hline & Sample ID & Taxonomy & Sample ID & Taxonomy & Sample ID & Taxonomy & Sample ID & Taxonomy \\
\hline & ESP_N1.1 & Petasites palmatus & SSP_N1.1 & Rubus ursinus & GM_N1.1 & Poasp. & MSH_N1.1 & Anaphalis margaritaceae \\
\hline & ESP_N1.2 & Poasp. & SSP_N1.2 & Maianthemum racemosum & GM_N1.2 & Pyrolla picta & MSH_N1.2 & Rubus lasiococcus \\
\hline & ESP_N2.1 & Vicia nigricans ssp. gigantea & SSP_N2.1 & Vaccinium parvifolium & GM_N2.1 & Achlys triphylla & MSH_N2.1 & Poasp. \\
\hline & ESP_N2.2 & Conium maculatum & SSP_N2.2 & Rubus ursinus & GM_N2.2 & Vaccinium parvifolium & MSH_N2.2 & Anaphalis margaritaceae \\
\hline & ESP_N3.1 & Heracleum maximum & SSP_N3.1 & Vaccinium parvifolium & GM_N3.1 & Clintonia uniflora & MSH_N3.1 & Poasp. \\
\hline & ESP_N3.2 & Conium maculatum & SSP_N3.2 & Rubus ursinus & GM_N3.2 & Vaccinium parvifolium & MSH_N3.2 & Digitalis purpurea \\
\hline & ESP_N4.1 & Petasites palmatus & SSP_N4.1 & Rubus ursinus & GM_N4.1 & Arctostaphylos nevadensis & MSH_N4.1 & Anaphalis margaritaceae \\
\hline & ESP_N4.2 & Vicia nigricans ssp. gigantea & SSP_N4.2 & Vaccinium parvifolium & GM_N4.2 & Vaccinium parvifolium & MSH_N4.2 & Rubus parvifolius \\
\hline & ESP_N5.1 & Petasites palmatus & SSP_N5.1 & Rubus ursinus & GM_N5.1 & Clintonia uniflora & MSH_N5.1 & Rubus parvifolius \\
\hline & ESP_N5.2 & Poasp. & SSP_N5.2 & Asarum caudatum & GM_N5.2 & Achlys triphylla & MSH_N5.2 & Sisymbrium officinale \\
\hline & ESP_N6.1 & Poasp. & SSP_N6.1 & Asarum caudatum & GM_N6.1 & Vaccinium parvifolium & MSH_N6.1 & Hypochaeris radicata \\
\hline & ESP_N6.2 & Cirsium sp. & SSP_N6.2 & Cornus unalaschkensis & GM_N6.2 & Abies grandis & MSH_N6.2 & Alnus viridis ssp. sinuata \\
\hline & ESP_N7.1 & Petasites palmatus & SSP_N7.1 & Asarum caudatum & GM_N7.1 & Achlys triphylla & MSH_N7.1 & Sisymbrium officinale \\
\hline & ESP_N7.2 & Poasp. & SSP_N7.2 & Pteridium aquilinium & GM_N7.2 & Maianthemum racemosum & MSH_N7.2 & Vaccinium parvifolium \\
\hline & ESP_N8.1 & Poasp. & SSP_N8.1 & Asarum caudatum & GM_N8.1 & Cornus unalaschkensis & MSH_N8.1 & Sisymbrium officinale \\
\hline & ESP_N8.2 & Vicia nigricans ssp. gigantea & SSP_N8.2 & Pteridium aquilinium & GM_N8.2 & Blechnum spicant & MSH_N8.2 & Castilleja miniata \\
\hline & ESP_N9.1 & Equisetum telmateia & SSP_N9.1 & Pteridium aquilinium & GM_N9.1 & Berberis nervosa & MSH_N9.1 & Anaphalis margaritaceae \\
\hline & ESP_N9.2 & Conium maculatum & SSP_N9.2 & Lapsana communis & GM_N9.2 & Trillium ovatum & MSH_N9.2 & Sorbus sitchensis \\
\hline & ESP_N10.1 & Poasp. & SSP_N10.1 & Rubus ursinus & GM_N10.1 & Blechnum spicant & MSH_N10.1 & Hypochaeris radicata \\
\hline & ESP_N10.2 & Conium maculatum & SSP_N10.2 & Asarum caudatum & GM_N10.2 & Achlys triphylla & MSH_N10.2 & Rubus parvifolius \\
\hline
\end{tabular}


Appendix B. PCR primers, reaction concentrations and cycling parameters for each locus utilized in the phylogenetic analysis of Chapter 4.

Locus

\begin{tabular}{|c|c|c|c|c|}
\hline & ITS & LSU & RPB1 & RPB2 \\
\hline \multicolumn{5}{|l|}{ Primers: } \\
\hline \multirow{5}{*}{ Forward primer } & ITS1F: & ITS3: & RPB2-5F2: & RPB2-5F2: \\
\hline & (CTTGGTCATTTAGA & (GCATCGATGAAGA & (GGGGWGAYCAGAA & (GGGGWGAYCAGAAGAA \\
\hline & GGAAGTAA) & ACGCAGC) & GAAGGC) & GGC) \\
\hline & ITS4: & LR6: & RPB2-7cR: & RPB2-7cR: \\
\hline & (TCCTCCGCTTATTG & (CGCCAGTTCTGCTT & (CCCATRGCTTGYTT & (CCCATRGCTTGYTTRCCC \\
\hline Reverse primer & ATATGC) & ACC) & RCCCAT) & AT) \\
\hline Reference: & White et al. 1990 & White et al. 1990 & Matheny et al. 2002 & O'Donnell et al. 2007 \\
\hline \multicolumn{5}{|l|}{ Reaction mixture $(\mu \mathrm{L})$ : } \\
\hline PCR H2O & 7.75 & 7.75 & 5.75 & 5.75 \\
\hline GoTaq 2X Master Mix & 12.50 & 12.50 & 12.50 & 12.50 \\
\hline BSA $(10 \mathrm{mg} / \mathrm{mL})$ & 1.25 & 1.25 & 1.25 & 1.25 \\
\hline Each primer $(10 \mu \mathrm{M})$ & 1.25 & 1.25 & 1.25 & 1.25 \\
\hline Template DNA & 1.00 & 1.00 & 3.00 & 3.00 \\
\hline 1:10 dilution of DNA? & Yes & Yes & No & No \\
\hline
\end{tabular}

Cycling parameters:

\begin{tabular}{|c|c|c|c|}
\hline$\left(94^{\circ} \mathrm{C}: 3 \mathrm{~min}\right) \mathrm{x} 1$ & $\left(94^{\circ} \mathrm{C}: 3 \mathrm{~min}\right) \mathrm{x} 1$ & $\begin{array}{l}\left(95^{\circ} \mathrm{C}: 5 \mathrm{~min}\right) \mathrm{x} 1 \\
\left(95^{\circ} \mathrm{C}: 1 \mathrm{~min}, 60^{\circ} \mathrm{C}-\right.\end{array}$ & $\begin{array}{l}\left(95^{\circ} \mathrm{C}: 5 \mathrm{~min}\right) \mathrm{x} 1 \\
\left(95^{\circ} \mathrm{C}: 1 \mathrm{~min}, 60^{\circ} \mathrm{C}-1^{\circ} \mathrm{C}\right.\end{array}$ \\
\hline$\left(95^{\circ} \mathrm{C}: 1 \mathrm{~min}, 50^{\circ} \mathrm{C}: 1\right.$ & $\left(95^{\circ} \mathrm{C}: 1 \mathrm{~min}, 50^{\circ} \mathrm{C}: 1\right.$ & $1^{\circ} \mathrm{C} \mathrm{cycle}^{-1}: 1 \min , 72^{\circ}$ & $\left.\operatorname{cycle}^{-1}: 1 \mathrm{~min}, 72^{\circ} \mathrm{C}: 2 \mathrm{~min}\right)$ \\
\hline $\left.\min , 72^{\circ} \mathrm{C}: 1 \mathrm{~min}\right) \times 35$ & $\left.\min , 72^{\circ} \mathrm{C}: 2 \min \right) \times 35$ & $\mathrm{C}: 2 \mathrm{~min}) \times 10$ & $\mathrm{x} 10$ \\
\hline$\left(72^{\circ} \mathrm{C}: 10 \mathrm{~min}\right) \mathrm{x} 1$ & $\left(72^{\circ} \mathrm{C}: 10 \mathrm{~min}\right) \mathrm{x} 1$ & $\begin{array}{l}\left(95^{\circ} \mathrm{C}: 1 \mathrm{~min}, 50^{\circ} \mathrm{C}: 1\right. \\
\left.\min , 72^{\circ} \mathrm{C}: 2 \mathrm{~min}\right) \times 35\end{array}$ & $\begin{array}{l}\left(95^{\circ} \mathrm{C}: 1 \mathrm{~min}, 50^{\circ} \mathrm{C}: 1 \mathrm{~min},\right. \\
\left.72^{\circ} \mathrm{C}: 2 \mathrm{~min}\right) \times 35\end{array}$ \\
\hline $4^{\circ} \mathrm{C}$ hold & $4^{\circ} \mathrm{C}$ hold & $\left(72^{\circ} \mathrm{C}: 5 \mathrm{~min}\right) \times 1$ & $\left(72^{\circ} \mathrm{C}: 5 \mathrm{~min}\right) \times 1$ \\
\hline & & $4^{\circ} \mathrm{C}$ hold & $4^{\circ} \mathrm{C}$ hold \\
\hline
\end{tabular}


Appendix C. Names and accession numbers of the taxa and their respective loci utilized in the narrow analysis of Chapter 4.

\begin{tabular}{|c|c|c|c|c|}
\hline Genus & Species & Strain & ITS & LSU \\
\hline Alatospora & acuminata & CCM F-02383 & AY204587 & $\mathrm{KC} 834018$ \\
\hline Alatospora & constricta & CCM F-11302 & KC834040 & KC834017 \\
\hline Alatospora & pulchella & CCM F-502 & KC834039 & KC834019 \\
\hline Catenospora & carrollii & BSY 0586 & TBD & TBD \\
\hline Catenospora & gilkeyae & BSY 0044 & TBD & TBD \\
\hline Catenospora & loowitii & BSY 0251 & TBD & TBD \\
\hline Catenospora & polysticola & BSY 0163 & TBD & TBD \\
\hline Claussenomyces & olivaceus & G.M. 2015-04-23.1 & KY661433 & KY661433 \\
\hline Collophora & africana & CBS 120872 & GQ154570 & GQ154609 \\
\hline Collophora & capensis & CBS 120879 & GQ154571 & GQ154610 \\
\hline Collophora & rubra & CBS 121441 & GQ154551 & GQ154607 \\
\hline Cyclaneusma & minus & AFTOL-ID 1296 & KU170126 & FJ176868 \\
\hline Cyclaneusma & niveum & N219 & KF013555 & KF013638 \\
\hline Flagellospora & leucorhynchos & CCM F-14183 & KC834049 & $\mathrm{KC} 834025$ \\
\hline Gelatinomyces & siamensis & KKUK1 & JX219379 & JX219381 \\
\hline Geoglossum & glabrum & ILLS 61038 & JQ256420 & JQ256436 \\
\hline Geoglossum & nigritum & AFTOL-ID 56 & DQ491490 & AY544650 \\
\hline Glutinoglossum & glutinosum & ILLS 64448 & КР690094 & КР690106 \\
\hline Glutinoglossum & heptaseptatum & ILLS 63754 & NR_132024 & $\mathrm{KC} 222143$ \\
\hline Gorgomyces & honrubiae & CCM F-12003 & KC834057 & KC834028 \\
\hline Leotia & lubrica & ZW-Geo59-Clark & AY789360 & AY789359 \\
\hline Marthamyces & desmoschoeni & PRJ R908 & KJ606679 & KJ606673 \\
\hline Microglossum & clavatum & SAV F-11276 & KX382864 & KX382864 \\
\hline Microglossum & griseoviride & SAV 9920 & КC595249 & KC595250 \\
\hline Microglossum & truncatum & LE 291847 & KX382863 & KX382871 \\
\hline Microglossum & viride & SAV 10249 & KC595253 & KC595254 \\
\hline Naemacyclus & culmigenus & TNS-F41728 & AB745435 & AB745437 \\
\hline Thuemenidium & atropurpureum & ILLS 61044 & JQ256427 & JQ256441 \\
\hline Trichoglossum & hirsutum & AFTOL-ID 64 & DQ491494 & AY544653 \\
\hline Trichoglossum & octopartitum & ILLS 67356 & KC222134 & $\mathrm{KC} 222147$ \\
\hline
\end{tabular}


Appendix D. Names and accession numbers of the taxa and their respective loci utilized in the wide analysis of Chapter 4.

\begin{tabular}{|c|c|c|c|c|c|c|c|}
\hline Genus & Species & Strain & SSU & ITS & LSU & RPB1 & RPB2 \\
\hline Acephala & applanata & CBS 109321 & KT259197 & NR_119482.1 & KT225544 & KT225541 & - \\
\hline Allantophomopsiella & pseudotsugae & CBS 322.53 & & JN033384 & JN086687 & - & JN086839 \\
\hline Ascocoryne & sarcoides & NRRL 50072 & AIAA01000117 & AIAA01000117 & AIAA01000117 & AIAA01000134 & AIAA01000122 \\
\hline Botrytis & cinerea & B05.10 & AAID02000582 & AAID02000582 & AAID02000582 & AAID02000880 & AAID02000899 \\
\hline Bulgaria & inquinans & AFTOL-ID 916 & DQ471008 & KJ663831 & DQ470960 & DQ471152 & DQ470910 \\
\hline Cadophora & malorum & Mo12 & FKJQ01000513 & FKJQ01000513 & FKJQ01000513 & FKJQ01000107 & FKJQ01000057 \\
\hline Cairneyella & variabilis & VPRI 42388 & AYLM01000747 & AYLM01000747 & AYLM01000747 & AYLM01000234 & AYLM01000538 \\
\hline Catenospora & carrollii & BSY 0586 & TBD & TBD & TBD & TBD & TBD \\
\hline Catenospora & gilkeyae & BSY 0044 & TBD & TBD & TBD & TBD & TBD \\
\hline Catenospora & loowitii & BSY 0251 & TBD & TBD & TBD & TBD & TBD \\
\hline Catenospora & polysticola & BSY 0163 & TBD & TBD & TBD & TBD & TBD \\
\hline Cenangiopsis & quercicola & KL174 & KX090862 & LT158425 & KX090811 & KX090760 & KX090713 \\
\hline Cenangium & ferruginosum & KL390 & KX090892 & LT158471 & KX090840 & - & KX090739 \\
\hline Chlorociboria & aeruginascens & IHIA39 & NCSK02000120 & NCSK02000120 & NCSK02000120 & NCSK02000115 & NCSK02000008 \\
\hline Chlorociboria & aeruginosa & AFTOL-ID 151 & AY544713 & DQ491501 & AY544669 & DQ471125 & DQ470886 \\
\hline Chlorociboria & halonata & D1553 & JN939861 & JN943470 & JN939936 & JN985210 & JN985510 \\
\hline Chlorociboria & spathulata & D1822 & JN939868 & JN943463 & JN939923 & JN985217 & JN985530 \\
\hline Ciborinia & camelliae & ICMP 19812 & LGKQ01001007 & LGKQ01001007 & LGKQ01001007 & LGKQ01000752 & LGKQ01000112 \\
\hline Coccomyces & dentatus & AFTOL-ID 147 & AY544701 & DQ491499 & AY544657 & - & DQ247789 \\
\hline Coccomyces & strobi & AFTOL-ID 1250 & DQ471027 & - & DQ470975 & DQ471173 & DQ470929 \\
\hline Cudonia & confusa & C314 & - & KC833165 & KC833216 & - & KC833300 \\
\hline Cudoniella & clavus & AFTOL-ID 166 & - & DQ491502 & DQ470944 & DQ471128 & DQ470888 \\
\hline Dermea & acerina & CBS 161.38 & DQ247809 & AF141164 & DQ247801 & DQ471164 & DQ247791 \\
\hline Diplocarpon & rosae & DortE4 & MVNX01000551 & MVNX01000551 & MVNX01000551 & MVNX01000022 & MVNX01000026 \\
\hline Diplolaeviopsis & cf. ranula & Diedrich 16989 & KX090896 & KJ559532 & KJ559554 & KX090790 & 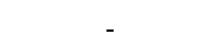 \\
\hline Encoelia & furfuracea & KL107 & KX090850 & LT158416 & KX090798 & KX090749 & KX090701 \\
\hline Encoelia & heteromera & KL164 & KX090861 & - & KX090809 & KX090758 & KX090712 \\
\hline Encoeliopsis & rhododendri & KL118 & KX090853 & - & KX090801 & KX090750 & KX090704 \\
\hline Geoglossum & glabrum & ILLS 61038 & - & JQ256420 & JQ256436 & KC222164 & - \\
\hline Geoglossum & nigritum & AFTOL-ID 56 & AY544694 & DQ491490 & AY544650 & DQ471115 & DQ470879 \\
\hline Glarea & lozoyensis & ATCC 20868 & ALVE01000196 & ALVE01000196 & ALVE01000196 & ALVE01000020 & ALVE01000147 \\
\hline
\end{tabular}


Appendix D. Continued

\begin{tabular}{|c|c|c|c|c|c|c|c|}
\hline Genus & Species & Strain & SSU & ITS & LSU & RPB1 & RPB2 \\
\hline Glutinoglossum & glutinosum & ILLS 64448 & - & KP690094 & KP690106 & KP690128 & - \\
\hline Glutinoglossum & heptaseptatum & ILLS 63754 & - & NR_132024 & KC222143 & $\mathrm{KC} 222172$ & - \\
\hline Graddonia & coracina & ILLS 60941 & - & JQ256423 & JN012009 & KC222173 & - \\
\hline Hyaloscypha & albohyalina var. spiralis & KUS-F52652 & - & JN033426 & JN086729 & - & JN086870 \\
\hline Hyaloscypha & aureliella & KUS-F52070 & - & JN033394 & JN086697 & - & JN086848 \\
\hline Hyaloscypha & hepaticola & M171 & EU940045 & JN943612 & EU940118 & JN985234 & - \\
\hline Hyaloscypha & vitreola & M39 & EU940079 & JN943613 & EU940155 & JN985240 & - \\
\hline Hymenoscyphus & fraxineus & CBS 133217 & LLCC01001145 & LLCC01001145 & LLCC01001145 & LLCC01000094 & LLCC01000239 \\
\hline Hymenoscyphus & scutula & CBS 480.97 & LKTO01001219 & LKTO01001219 & LKTO01001219 & LKTO01000157 & LKTO01000184 \\
\hline Ionomidotis & fulvotingens & KL231 & KX090870 & - & KX090819 & KX090765 & KX090719 \\
\hline Lachnum & virgineum & AFTOL-ID 49 & AY544688 & DQ491485 & AY544646 & DQ842030 & DQ470877 \\
\hline Lambertella & corni-maris & TNS-F40083 & - & AB926069 & AB926139 & - & AB926184 \\
\hline Lambertella & subrenispora & AFTOL-ID 1262 & - & AB926097 & DQ470978 & DQ471176 & DQ470930 \\
\hline Leotia & atrovirens & $3 \mathrm{Can}$ & - & AY144566 & - & - & AY144531 \\
\hline Leotia & lubrica & AFTOL-ID 1 & NG_013133 & DQ491484 & AY544644 & DQ471113 & DQ470876 \\
\hline Leotia & $v i s \cos a$ & 1Chi & - & AY144537 & - & - & AY144502 \\
\hline Leuconeurospora & pulcherrima & AFTOL-ID 1397 & FJ176828 & KF049206 & FJ176884 & FJ238440 & KJ755491 \\
\hline Loramyces & macrosporus & AFTOL-ID 913 & DQ471005 & NR_138379 & DQ470957 & KP965570 & DQ470907 \\
\hline Marssonina & brunnea f. sp. multigermtubi & MB_m1 & AFXC01002388 & AFXC01002388 & AFXC01002388 & AFXC01000716 & AFXC01001127 \\
\hline Meliniomyces & bicolor & E K444 & LXPI01000297 & LXPI01000277 & LXPI01000277 & LXPI01000082 & LXPI01000022 \\
\hline Meliniomyces & variabilis & F L207 & LXPR01000262 & LXPR01000262 & LXPR01000261 & LXPR01000091 & LXPR01000026 \\
\hline Microglossum & clavatum & SAV F-11276 & - & KX382864 & KX382864 & - & KX382884 \\
\hline Microglossum & griseoviride & SAV 9920 & - & KC595249 & KC595250 & - & KX382872 \\
\hline Microglossum & olivaceum & KL220 & KX090868 & - & KX090817 & KX090764 & KX090718 \\
\hline Microglossum & rufum & AFTOL-ID 1292 & DQ471033 & - & DQ470981 & DQ471179 & DQ470933 \\
\hline Microglossum & truncatum & LE 291847 & - & KX382863 & KX382871 & - & KX382876 \\
\hline Microglossum & viride & SAV 10249 & - & KC595253 & KC595254 & - & KX382873 \\
\hline Mollisia & cinerea & AFTOL-ID 76 & DQ470990 & DQ491498 & DQ470942 & DQ471122 & DQ470883 \\
\hline Myriosclerotinia & curreyana & LMK 759 & NGKJ01000264 & NGKJ01000264 & NGKJ01000264 & NGKJ01000004 & NGKJ01000019 \\
\hline Myxotrichum & deflexum & CBS 228.61 & AB015777 & LN833542 & AB040689 & - & LN833563 \\
\hline Neobulgaria & pura & AFTOL-ID 1259 & AF222533 & - & FJ176865 & FJ238434 & FJ 238350 \\
\hline Neofabraea & malicorticis & AFTOL-ID 149 & AY544706 & NR_144926 & AY544662 & DQ471124 & DQ470885 \\
\hline
\end{tabular}




\begin{tabular}{|c|c|c|c|c|c|c|c|}
\hline $\begin{array}{c}\text { Appendix D. Co } \\
\text { Genus }\end{array}$ & Species & Strain & SSU & ITS & LSU & RPB1 & RPB2 \\
\hline Oidiodendron & maius & $\mathrm{Zn}$ & JMDP01000383 & JMDP01000384 & JMDP01000382 & JMDP01000121 & JMDP01000031 \\
\hline Parafabraea & eucalypti & CBS 124810 & - & KR859091 & KR858882 & - & KR859331 \\
\hline Perrotia & populina & KL120 & KX090854 & - & KX090802 & KX090751 & KX090705 \\
\hline Pezicula & carpinea & AFTOL-ID 938 & DQ471016 & - & DQ470967 & DQ842032 & DQ479934 \\
\hline Phacidium & lacerum & AFTOL-ID 1253 & DQ471028 & KJ663841 & DQ470976 & DQ471174 & KJ663923 \\
\hline Phaeohelotium & epiphyllum & TNS-F40042 & & AB926061 & AB926130 & - & AB926219 \\
\hline Phialocephala & scopiformis & CBS 120377 & LKNI01000331 & LKNI01000331 & LKNI01000331 & LKNI01000010 & LKNI01000089 \\
\hline Phlyctema & vagabunda & CBS 109875 & - & KR859275 & KR859069 & - & KR859346 \\
\hline Potebniamyces & pyri & AFTOL-ID 744 & DQ470997 & DQ491510 & DQ470949 & DQ471142 & DQ470900 \\
\hline Pseudeurotium & zonatum & AFTOL-ID 1912 & DQ471040 & KJ755522 & DQ470988 & DQ471186 & KJ755494 \\
\hline Pseudogymnoascus & destructans & $20631-21$ & XR_001167164 & EU884921 & XR_001167165 & XM_012890932 & XM_012886952 \\
\hline Pseudogymnoascus & sp. VKM F-3557 & VKM F-3557 & JPJS01003119 & JPJS01003284 & JPJS01003284 & JPJS01001441 & JPJS01001480 \\
\hline Rhynchosporium & lolii & $15 \operatorname{lp} 11$ & KU844336 & KU844336 & KU844336 & - & KU844339 \\
\hline Rutstroemia & bulgarioides & KL98 & KX090848 & LT158483 & KX090797 & - & KX090700 \\
\hline Rutstroemia & echinophila & CBS 111548 & JWJA01000014 & JWJA01000014 & JWJA01000014 & JWJA01004570 & JWJA01005778 \\
\hline Rutstroemia & sydowiana & CBS 115975 & JWJB01000045 & JWJB01000045 & JWJB01000045 & JWJB01001304 & JWJB01010742 \\
\hline Sclerencoelia & fraxinicola & KL156 & KX090857 & LT158420 & KX090805 & KX090755 & KX090708 \\
\hline Sclerotinia & sclerotiorum & 1980 UF-70 & AAGT01000678 & AAGT01000678 & AAGT01000678 & AAGT01000243 & AAGT01000031 \\
\hline Skyttea & radiatilis & SK91 & - & KJ559538 & KJ559560 & KX090791 & KX090742 \\
\hline Spathularia & flavida & H656 & - & KC833110 & KC833255 & - & KC833336 \\
\hline Thamnogalla & crombiei & Diedrich 17553 & KJ559583 & KJ559535 & KJ559557 & - & KX090743 \\
\hline Thelebolus & ellipsoideus & AFTOL-ID 5005 & DQ067574 & AY957550 & FJ176895 & FJ238445 & FJ238378 \\
\hline Thelebolus & globosus & AFTOL-ID 5016 & FJ176851 & DQ028268 & FJ176905 & FJ238446 & FJ238385 \\
\hline Thuemenidium & atropurpureum & ILLS 61044 & - & JQ256427 & JQ256441 & $\mathrm{KC} 222176$ & - \\
\hline Trichoglossum & hirsutum & AFTOL-ID 64 & AY544697 & DQ491494 & AY544653 & DQ471119 & DQ470881 \\
\hline Trichoglossum & octopartitum & ILLS 67356 & - & KC222134 & $\mathrm{KC} 222147$ & $\mathrm{KC} 222181$ & - \\
\hline Trochila & laurocerasi & KL336 & KX090887 & LT158460 & KX090835 & KX090780 & KX090734 \\
\hline Tryblidiopsis & pinastri & AFTOL-ID 1319 & DQ471035 & JF793678 & DQ470983 & DQ471181 & DQ470935 \\
\hline Varicosporium & elodeae & AU_CRYP05 & JN938734 & JN995640 & JN941371 & JN985043 & - \\
\hline Velutarina & alpestris & KL378 & KX090891 & LT158470 & KX090839 & KX090786 & KX090738 \\
\hline Vibrissea & truncorum & AFTOL-ID 1322 & FJ176818 & EU434854 & FJ176874 & FJ238438 & FJ238356 \\
\hline
\end{tabular}

